BOSTON UNIVERSITY

GRADUATE SCHOOL OF ARTS AND SCIENCES

Dissertation

\title{
TOP QUARK MASS IN EVENTS WITH TWO CHARGED LEPTONS AT THE DO EXPERIMENT
}

by

\section{DANIEL BOLINE}

B.S., Physics, Michigan Technological University, 2002

Submitted in partial fulfillment of the

requirements for the degree of

Doctor of Philosophy 


\section{Approved by}

First Reader

Ulrich Heintz, Ph.D.

Professor of Physics

Second Reader

Robert Carey, Ph.D.

Associate Professor of Physics 


\section{ACKNOWLEDGMENTS}

I would like to thank: my Parents, for being supportive and providing me with very useful advise, love and support, my Advisor, Ulrich, for his patience, assistance and understanding, as well as Meenakshi Narain, for guiding me during my first days with DØ .

I'd also like to thank my fellow grad students and postdocs. Lorenzo Feligioni and Sarosh Fatakia who provided assistance during my first trips to Fermilab, also Shabnam Jabeen, Dookee Cho, Vivek Parihar, and Amithab Das. I'd like to thank my good friend Tuhin Roy. 


\title{
TOP QUARK MASS IN EVENTS WITH TWO CHARGED LEPTONS AT THE DO EXPERIMENT
}

\author{
(Order No. \\ ) \\ DANIEL BOLINE \\ Boston University, Graduate School of Arts and Sciences, 2010 \\ Major Advisor: Ulrich Heintz, Professor of Physics
}

\begin{abstract}
The top quark is the most massive observed fundamental subatomic particle, and at the Tevatron accelerator is produced mostly in top-antitop $(t \bar{t})$ quark pairs from the collisions of protons and anti-protons. Each top quark decays into a bottom quark and a W boson. The $\mathrm{W}$ boson can then decay into a pair of quarks, or into a charged lepton and a neutrino. The various decays can be broken up into three different channels based on the number of leptons from the decay of the $\mathrm{W}$ bosons: all-jets (with no leptons), lepton+jets (with one lepton), and dilepton (with two leptons). This dissertation will present a measurement of the top quark mass in the dilepton channel.

The dilepton channel is characterized by two leptons, two neutrinos and two b-quarks. The neutrinos are not directly observed, but their absence is felt as missing transverse momentum $\left(\not \not p_{T}\right)$ in the detector. The combination of two leptons and large $\not p_{T}$ produces an easily isolated signal, giving the dilepton channel a high signal over background ratio.

Having two neutrinos means that we cannot know what the transverse momenta of either neutrino is. This means that even if we knew the momenta of the leptons and b-quarks perfectly, we would be unable to reconstruct the mass of the top quark. This measurement gets around this problem by scanning over all possible values of the top mass, finding all consistent $t \bar{t}$ combinations, assigning a kinematic weight to each, and then adding the weights for each combination at a given possible top mass. The lepton momenta, jet momenta, and $\not p_{T}$ are only known to within some finite precision, so for a given top mass, I also vary each of these momenta within their resolutions and add the weights for a given possible top mass. After scanning over possible top masses, I choose
\end{abstract}


the top mass with the largest sum of weights $m_{t}^{\max }$ as an observable for the event. I then perform a template based likelihood fit of $m_{t}$ using $m_{t}^{\max }$. I analyze 322 candidate events collected by the $\mathrm{D} \varnothing$ detector, and obtain a top quark mass of:

$$
m_{t}=174.8 \pm 3.1 \mathrm{GeV}
$$




\section{Contents}

Contents vi vi vis

List of Tables $\quad$ xi

List of Figures xiii

List of Abbreviations $\quad$ xxi

1 Introduction 1

1.1 The Standard Model and the Top Quark . . . . . . . . . . . . . . 1

1.2 Top Quark Mass, Constraint on Higgs Mass . . . . . . . . . . . . . . . . . 3

1.3 Top Quark Pair Production and Decay . . . . . . . . . . . . . . . 3

1.3.1 Theoretical Predictions of Production Cross Section . . . . . . . 5

1.3.2 Measurement of Top Mass using Measured Cross Section . . . . . . 6

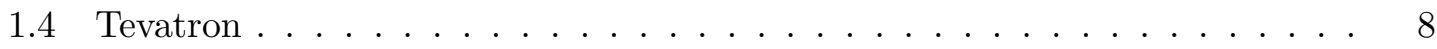

2 Detector $\quad 10$

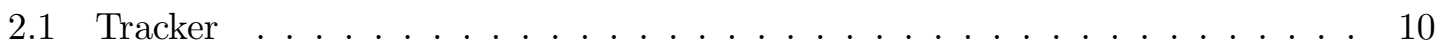

2.1.1 Silicon Microstrip Tracker . . . . . . . . . . . . . . . . . 11

2.1.2 Central Fiber Tracker . . . . . . . . . . . . . . . 12

2.2 Solenoid Magnet . . . . . . . . . . . . . . . . . . . . . . 14

2.3 Central and Forward Preshower . . . . . . . . . . . . . . . . 14

2.4 Calorimeter . . . . . . . . . . . . . . . . . . 15

2.4 .1 Inter Cryostat Detector f . . . . . . . . . . . . . 17

2.4.2 Noise in the Calorimeter . . . . . . . . . . . . . 18

2.5 Muon System . . . . . . . . . . . . . . . . . . . . . . 18

2.5.1 Toroid Magnet . . . . . . . . . . . . . . 18 


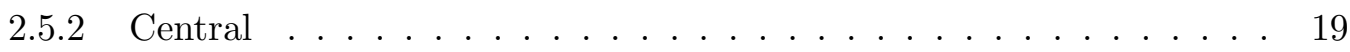

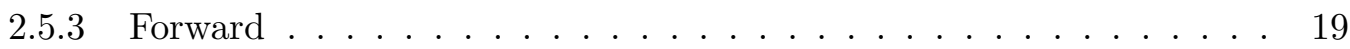

2.6 Luminosity Monitor . . . . . . . . . . . . . . . . . . . . . 20

2.7 Trigger . . . . . . . . . . . . . . . . . . . 20

2.7 .1 Level $1 \ldots \ldots \ldots \ldots \ldots$. . . . . . . . . . . . . . . . 22

2.7 .2 Level $2 \ldots \ldots \ldots \ldots \ldots \ldots$

2.7 .3 Level $3 \ldots \ldots \ldots \ldots \ldots \ldots$

3 Object Reconstruction $\quad 27$

3.1 Track Reconstruction . . . . . . . . . . . . . . . . . 27

3.1.1 Motion of a Charged Particle in the DØ detector . . . . . . . . . 27

3.1 .2 Track Finding Algorithms . . . . . . . . . . . . . . . . 30

3.1.3 Track Momentum Resolution . . . . . . . . . . . . . . . . . . . 31

3.2 EM Cluster Reconstruction . . . . . . . . . . . . . . . . . . 33

3.2 .1 Electromagnetic Showers . . . . . . . . . . . . . . 33

3.2.2 Electromagnetic Cluster Reconstruction . . . . . . . . . . . . . . . . 34

3.2 .3 Electron Energy Resolution . . . . . . . . . . . . . . . . 36

3.3 Jet Reconstruction . . . . . . . . . . . . . . . . . . . . 37

3.3 .1 Hadronic Showers . . . . . . . . . . . . . . . . . . . 37

3.3 .2 Jet Reconstruction Algorithm . . . . . . . . . . . . . . . . . . 38

3.3.3 Jet Energy Scale . . . . . . . . . . . . . . . . . . . . . 39

3.3.4 Single Pion Response . . . . . . . . . . . . . . . . . . . . 40

3.3.5 Sample Dependence Correction . . . . . . . . . . . . . . . . . . . 40

3.3.6 Jet Energy Resolution . . . . . . . . . . . . . . . . . . . 43

3.4 Muon Reconstruction . . . . . . . . . . . . . . . . . . 44

3.4.1 Muon Reconstruction Algorithm . . . . . . . . . . . . . . . . 44

3.4 .2 Muon Identification . . . . . . . . . . . . . . . 45

3.5 Missing Transverse Energy Reconstruction . . . . . . . . . . . . . . . . . 46

3.5.1 Missing Transverse Momentum Corrections . . . . . . . . . . . . . 47 
3.5.2 Missing Transverse Momentum Resolution, $\not p_{T}^{\prime}$ Significance. . . . . . 47

4 DØ Data 49

4.1 Time Periods . . . . . . . . . . . . . . . . . . . . . . 49

4.2 Data Quality . . . . . . . . . . . . . . . . 50

5 Monte Carlo $\quad 51$

5.1 Event Generators: ALPGEN, PYTHIA . . . . . . . . . . . . . . 51

5.1 Parton Jet Matching, Heavy Flavor Removal . . . . . . . . . . . . . 51

5.2 Monte Carlo Production Chain . . . . . . . . . . . . . . . . . . 52

5.3 Monte Carlo Corrections . . . . . . . . . . . . . . . . . . . . 53

5.3.1 Z Boson Transverse Momentum Reweighting . . . . . . . . . . 53

5.3.2 Beam Longitudinal Position Reweighting . . . . . . . . . . . 53

5.3 .3 Instantaneous Luminosity Reweighting . . . . . . . . . . . . . . . . 54

5.3.4 Electron and Muon Identification Efficiency Scale Factor . . . . . . . 54

5.3.5 Jet Identification Efficiency Correction through Jet Removal . . . . 55

5.3 .6 Electron Energy Smearing . . . . . . . . . . . . . . . . 55

5.3 .7 Muon Momentum Smearing . . . . . . . . . . . . . . . 55

5.3 .8 Jet Smearing, Shifting and Removal . . . . . . . . . . . . . 55

$\begin{array}{lll}6 & \text { Event Selection } & 57\end{array}$

6.1 Selections for Analysis . . . . . . . . . . . . . . . 57

$6.1 .1 e \mu$ channel selection . . . . . . . . . . . . . 57

6.1 .2 ee channel selection . . . . . . . . . . . . . . . 58

$6.1 .3 \mu \mu$ channel selection . . . . . . . . . . . . . . . . 60

6.1 .4 Yield for Run IIa . . . . . . . . . . . . . . . . . . . . . 63

6.1.5 Determination of fake rate . . . . . . . . . . . . . 64

$\begin{array}{lll}7 & \text { Mass Measurement } & 68\end{array}$

7.1 Kinematic Reconstruction . . . . . . . . . . . . . . . . . 68

7.2 Weighting . . . . . . . . . . . . . . . . . . 69

7.3 Smearing of Object Momenta . . . . . . . . . . . . . . 69 
7.4 Template Fit for Top Mass . . . . . . . . . . . . . . . . . . 70

7.5 Performance of Method on Simulated Data . . . . . . . . . . . . . . . 71

7.6 Result from DØ Data . . . . . . . . . . . . . . . . . . . 74

7.7 Mass Measurements Split into Channels . . . . . . . . . . . . . . . 75

7.7.1 Run IIa Mass Measurement per Channel . . . . . . . . . . . . . . . . 75

7.7.2 Run IIb Mass Measurement per Channel _ . . . . . . . . . . . . . . 79

8 Systematic Uncertainties $\quad 82$

8.1 Jet Reconstruction . . . . . . . . . . . . . . . . . . . . 82

8.1 .1 Jet Energy Scale . . . . . . . . . . . . . . . . . 82

8.1.2 b/l Response and Sample-Dependent Response . . . . . . . . . . . . 83

8.1 .3 b-Fragmentation . . . . . . . . . . . . 83

8.2 Signal Modeling . . . . . . . . . . . . . . . . . . . . . . . 84

8.2 .1 Hard Scatter . . . . . . . . . . . . . . . . . . . . 84

8.2 .2 Color Reconnection . . . . . . . . . . . . . . . . . 84

8.2 .3 Particle Showering Tune . . . . . . . . . . . . . . . . . 84

8.2.4 Higher Order Effects . . . . . . . . . . . . . . . . . . 84

8.2.5 Hadronization . . . . . . . . . . . . . . . . . . 85

8.2 .6 Initial and Final State Radiation . . . . . . . . . . . . . . . . . 85

8.2 .7 Multiple Interactions . . . . . . . . . . . . . . . . . . 85

8.2.8 Parton Distribution Function . . . . . . . . . . . . . . . 85

8.3 Detector Model . . . . . . . . . . . . . . . . . . . . 86

8.3 .1 Jet Energy Resolution . . . . . . . . . . . . . . . . . . 86

8.3 .2 Jet Identification Efficiency . . . . . . . . . . . . . . . . 86

8.3 .3 Electron, Muon Identification . . . . . . . . . . . . . . . . 86

8.3.4 Z-position Profile of Primary Event Vertex . . . . . . . . . . 86

8.4 Lepton Momentum Scale and Resolution . . . . . . . . . . . . . . . 87

8.4.1 Muon Momentum Resolution . . . . . . . . . . . . . . . . 87

8.4 .2 Electron Energy Resolution . . . . . . . . . . . . . 87 
8.4 .3 Muon Momentum Scale . . . . . . . . . . . . . . . . . . 87

8.4.4 Electron Momentum Scale . . . . . . . . . . . . . . . . 87

8.5 Background Model . . . . . . . . . . . . . . . . . . 88

8.5.1 Background Normalization . . . . . . . . . . . . . . 88

8.5.2 Physics Background Model . . . . . . . . . . . . . . 88

8.5.3 Fake Background Model . . . . . . . . . . . . . . . . . . 88

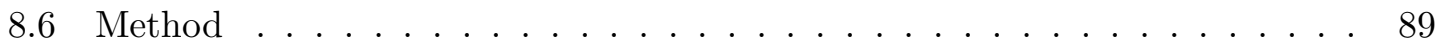

8.6 .1 Template Statistics . . . . . . . . . . . . . . . . 89

8.6.2 Ensemble Statistics . . . . . . . . . . . . . . . . . . 89

8.7 Systematic Uncertainties per Channel . . . . . . . . . . . . . . . . . . . . . 91

8.8 Combination of Systematic Uncertainties for Run IIa and Run IIb . . . . . 93

9 Conclusion $\quad 94$

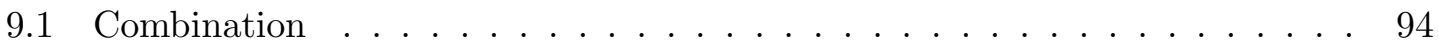

9.2 Comparison with Other Measurements, Impact . . . . . . . . . . . . 95

9.3 Prospects for Top Mass at the Tevatron . . . . . . . . . . . . . . . . 95

A Combining Measurements with Correlated Errors: BLUE Method $\quad 97$

B Jet Transfer Function Parameters for RunIIb $\quad 98$

C Control Plots for $e \mu, e e$ and $\mu \mu$ channels in RunIIb 100

List of Journal Abbreviations 114

$\begin{array}{ll}\text { Bibliography } & 115\end{array}$

$\begin{array}{ll}\text { Curriculum Vitae } & 121\end{array}$ 


\section{List of Tables}

3.1 Parameters for the Muon Resolution. . . . . . . . . . . . . . . . . . . 33

3.2 Parameters for the Electron Resolution [1]. . . . . . . . . . . . . . . 36

3.3 Parameters for the Jet Energy Resolution. . . . . . . . . . . . . . . . . . 44

5.1 Cross Sections for Monte Carlo samples. . . . . . . . . . . . . . . . 56

6.1 Cumulative efficiency and data over Monte Carlo scale factors for $t \bar{t} \rightarrow l \bar{l} \nu \bar{\nu} b \bar{b}$ signal with $m_{t}=172.5 \mathrm{GeV}$ in $e \mu$ channel in Run IIb. . . . . . . . . . . . . 59

6.2 Yield for $e \mu$ selection in Run IIb. Note: all selections have $L_{e} \geq 0.85$ requirement. . . . . . . . . . . . . . . . . . . 59

6.3 Cut flow efficiency for $t \bar{t} \rightarrow l \bar{l} \nu \bar{\nu} b \bar{b}$ signal with $m_{t}=172.5 \mathrm{GeV}$ in $e e$ channel in Run IIb. . . . . . . . . . . . . . . . . . . . . . . . 60

6.4 Yield for ee selection in Run IIb. . . . . . . . . . . . . . . . . . . . . . . 61

6.5 Cut flow efficiency for $t \bar{t} \rightarrow l \bar{l} \nu \bar{\nu} b \bar{b}$ signal with $m_{t}=172.5 \mathrm{GeV}$ in $\mu \mu$ channel in Run IIb. . . . . . . . . . . . . . . . . . . . . . . . 62

6.6 Yield for $\mu \mu$ selection in Run IIb. . . . . . . . . . . . . . . . . . . . 62

6.7 Expected and observed event yields for the dilepton channels in Run IIa. . . 63

6.8 Expected and observed event yields for the dilepton channels in Run IIb. . 63

6.9 Expected and observed event yields for the dilepton channels for full Run II. 63

8.1 Number of fake events for efficiencies obtained with inclusive jets, and $\geq 2$ jets, see Fig. 6.1-6.4 for dependence of efficiencies on jet multiplicity. . . . . 88

8.2 Summary of uncertainties. . . . . . . . . . . . . . . . . 90

8.3 Summary of uncertainties for Run IIa, combined, dilepton, lepton+track channels separately. . . . . . . . . . . . . . . . . . . 91 


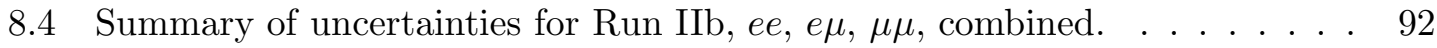

8.5 Combination of uncertainties for Run IIa Run IIb. . . . . . . . . . . . . . . 93 


\section{List of Figures}

1.1 The larger dashed red band shows the $68 \%$ confidence limit (CL) for $m_{W}$ vs. $m_{t}$ determined using data from the first run of LEP and SLD, while the blue line shows the same for the second run of LEP and the Tevatron, the green band shows corresponding values of $m_{H}$ between 113 and $1000 \mathrm{GeV}$ [2]. 4

1.2 Decay Channels for Top Quark Pair Production. . . . . . . . . . . . 6

1.3 Theory cross section vs. measured cross section [3], the grey band gives the error on the measured cross section. . . . . . . . . . . . . . 7

1.4 Diagram of Tevatron Accelerator Chain(left), Integrated Luminosity as a Function of Time at the Tevatron(right). . . . . . . . . . . . . 9

2.1 Diagram of current DØdetector (left) and central detector (right) [4] . . . . 11

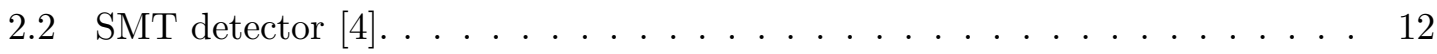

2.3 Layout of CFT and CPS axial fibers [4] . . . . . . . . . . . . . 13

2.4 View of the $\mathrm{D} \varnothing$ magnetic field showing both the solenoid and toroid magnets at full current $[4] \ldots \ldots \ldots \ldots \ldots$. . . . . . . . . . . . . . . . 15

2.5 Diagram of calorimeter cell(left), Charged particle traveling through solenoid magnetic field(right) $[4] \ldots \ldots \ldots \ldots$. . . . . . . . . . . . . 17

2.6 Diagram of current DØtrigger system [5]. . . . . . . . . . . . . . . . 21

2.7 Schematic diagram of sliding windows algorithm for jets. Roi stands for region of interest and is the $2 \times 2$ red squares in the center, the sum of the towers in this region is maximized. The $4 \mathrm{x} 4$ region just outside the Roi is used to determine the final $E_{T}$ of the cluster, while the $6 \mathrm{x} 6$ region outside that is used to remove overlapping clusters [5]. . . . . . . . . . . . . . 23 
3.1 Charged particle traveling through solenoid magnetic field. . . . . . . . . . 28

3.2 Energy loss for muons traveling through copper $[6] \ldots \ldots$. . . . . . . . 30

3.3 Extension of track hypothesis to new layer of detector [7]. . . . . . . . . 31

3.4 Upper left: Track hypotheses. Upper right: Line in $\rho, \phi$ for single hypothesis. Lower left: Several hypotheses. Lower right: Histogramming of hypotheses [8]. 32

3.5 Comparison of single pion response derived from data (full circles), and from the fit to the jet response (thick solid line). Quark jet fraction in $\gamma+$ jet events as a function of photon energy [9]. . . . . . . . . . . . . . . . . . 41

$3.6 k_{t \bar{t}}^{l}$ for light jets in various $|\eta|$ regions $[10] . \ldots \ldots \ldots \ldots \ldots$

$3.7 k_{t \bar{t}}^{b}$ for b jets in various $|\eta|$ regions $[10] \ldots \ldots \ldots \ldots \ldots$

3.8 Jet Energy Resolution for Data and Monte Carlo in the CC(left) and not CC(right) $[11] . \ldots \ldots \ldots \ldots \ldots \ldots \ldots$

3.9 Resolution of $\not p_{T}$ as a function of $\sqrt{U E}$ in data (red) and Monte Carlo (blue). 48

5.1 Data vs MC for $N_{\text {jet } 20}=1$ for events with no zpt-reweighting, the inclusive reweighting, and the final per-jet reweighting, with Run IIb data/MC [12].

5.2 Data vs MC for $N_{\text {jet } 20} \geq 2$ for events with no zpt-reweighting, the inclusive reweighting, and the final per-jet reweighting, with Run IIb data/MC [12]. 54

$6.1 \epsilon_{\text {real }}^{e}$ determination from $Z \rightarrow e e$, versus $m_{e e}$, dependence on $N_{j e t s}, p_{T}^{e} \ldots .66$

$6.2 \epsilon_{\text {fake }}^{e}$ determination from anti-isolated $e \mu$ sample, versus $\not_{T}$, dependence on

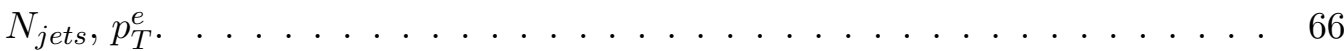

$6.3 \epsilon_{\text {real }}^{\mu}$ determination from $Z \rightarrow \mu \mu$, versus $m_{\mu \mu}$, dependence on $N_{\text {jets }}, p_{T}^{\mu}$. . 67

$6.4 \epsilon_{\text {fake }}^{\mu}$ determination from anti-isolated $\mu \mu$ sample, versus $\not p_{T}$, dependence on $N_{j e t s}, p_{T}^{\mu} \ldots \ldots \ldots \ldots \ldots \ldots \ldots \ldots \ldots \ldots \ldots \ldots \ldots \ldots \ldots \ldots$

7.1 Combined background templates for $e e, e \mu, \mu \mu$, e+track and $\mu+$ track in Run IIa. . . . . . . . . . . . . . . . . . . . . . . . 71

7.2 Background templates for $e e, e \mu$, and $\mu \mu$ in Run IIb. . . . . . . . . . . . 71

7.3 Templates from Monte Carlo events from $t \bar{t}$ decays to $e e$ for $m_{t}=150 \mathrm{GeV}$ (left), $172.5 \mathrm{GeV}$ (center), and $195 \mathrm{GeV}$ (right). . . . . . . . . 72 
7.4 Templates from Monte Carlo events from $t \bar{t}$ decays to $e \mu$ for $m_{t}=150 \mathrm{GeV}$ (left), $172.5 \mathrm{GeV}$ (center), and $195 \mathrm{GeV}$ (right) . . . . . . . . . . 72

7.5 Templates from Monte Carlo events from $t \bar{t}$ decays to $\mu \mu$ for $m_{t}=150 \mathrm{GeV}$ (left), $172.5 \mathrm{GeV}$ (center), and $195 \mathrm{GeV}$ (right). . . . . . . . . . . 72

7.6 Fitted mass distribution, pull distribution, and error distribution for 1000 ensembles with $m_{t}=172.5 \mathrm{GeV}$ for combination of channels. . . . . . . . . 73

7.7 Average fit mass, pull, and pull width versus input top quark mass for the ensemble tests for combination of channels. . . . . . . . . . . . . . 73

7.8 Plots of $-\ln L$ versus top quark mass for Run IIa (left) and Run IIb (right). 74

7.9 comparison of peak masses in data and Monte Carlo in Run IIa (left) Run IIb (center), and full Run II (right). . . . . . . . . . . . . . . . . . . . 74

7.10 Fitted mass distribution, error distribution, and pull distribution for 1000 ensembles with $m_{t}=175 \mathrm{GeV}$ in ee channel. . . . . . . . . . . . . . . 75

7.11 Average fit mass, pull, and pull width versus input top quark mass for the ensemble tests in ee channel. . . . . . . . . . . . . . . . . . . . . 76

7.12 Fitted mass distribution, error distribution, and pull distribution for 1000 ensembles with $m_{t}=175 \mathrm{GeV}$ in $e \mu$ channel. . . . . . . . . . . . . . 76

7.13 Average fit mass, pull, and pull width versus input top quark mass for the ensemble tests in $e \mu$ channel. . . . . . . . . . . . . . . . . . . . . . 76

7.14 Fitted mass distribution, error distribution, and pull distribution for 1000 ensembles with $m_{t}=175 \mathrm{GeV}$ in $\mu \mu$ channel. . . . . . . . . . . . . . 76

7.15 Average fit mass, pull, and pull width versus input top quark mass for the ensemble tests in $\mu \mu$ channel. . . . . . . . . . . . . . . . . 77

7.16 Fitted mass distribution, error distribution, and pull distribution for 1000 ensembles with $m_{t}=175 \mathrm{GeV}$ in $e+$ track channel. . . . . . . . . . . 77

7.17 Average fit mass, pull, and pull width versus input top quark mass for the ensemble tests in $e+$ track channel. . . . . . . . . . . . . . . . . . . 77 
7.18 Fitted mass distribution, error distribution, and pull distribution for 1000 ensembles with $m_{t}=175 \mathrm{GeV}$ in $\mu+$ track channel. . . . . . . . . . . . 77

7.19 Average fit mass, pull, and pull width versus input top quark mass for the ensemble tests in $\mu+$ track channel. . . . . . . . . . . . . . . . . 78

7.20 plots of $-\ln L$ versus top quark mass in the $e e, e \mu, \mu \mu, e+$ track and $\mu+$ track channels. . . . . . . . . . . . . . . . . 78

7.21 Fitted mass distribution, error distribution, and pull distribution for 1000 ensembles with $m_{t}=172.5 \mathrm{GeV}$ in ee channel. . . . . . . . . . . . . 79

7.22 Average fit mass, pull, and pull width versus input top quark mass for the ensemble tests in ee channel. . . . . . . . . . . . . . . . . . . . . 79

7.23 Fitted mass distribution, error distribution, and pull distribution for 1000 ensembles with $m_{t}=172.5 \mathrm{GeV}$ in $e \mu$ channel. . . . . . . . . . . . . . . 80

7.24 Average fit mass, pull, and pull width versus input top quark mass for the ensemble tests in $e \mu$ channel. . . . . . . . . . . . . . . . . . . 80

7.25 Fitted mass distribution, error distribution, and pull distribution for 1000 ensembles with $m_{t}=172.5 \mathrm{GeV}$ in $\mu \mu$ channel. $\ldots \ldots \ldots . . \ldots 80$

7.26 Average fit mass, pull, and pull width versus input top quark mass for the ensemble tests in $\mu \mu$ channel. . . . . . . . . . . . . . . . . . . . 80

7.27 Average RMS of fitted mass distributions for $e e, e \mu$, and $\mu \mu$ channels. . . . . 81

7.28 Plots of $-\ln L$ versus top quark mass and comparison of peak masses in data and Monte Carlo in the $e e, e \mu$, and $\mu \mu$ channels. . . . . . . . . . . 81

7.29 Variation in measured top mass in data due to removal of one event in ee, $e \mu$, and $\mu \mu$ channels. . . . . . . . . . . . .

8.1 Average fit mass versus input top quark mass for ensemble tests with the jet scale varied by $\pm 1 \sigma$.

8.2 Nominal fake templates compared with templates using $p_{T}$ dependent fake rate $(e e, e \mu, \mu \mu) \ldots \ldots \ldots \ldots \ldots \ldots$

8.3 Fake templates obtained from loose same-sign samples $(e e, e \mu, \mu \mu) . \quad \ldots . . \quad 89$ 
9.1 The world average top mass, along with the contributing measurements form $\mathrm{D} \varnothing$ and $\mathrm{CDF}[13] \ldots \ldots \ldots \ldots \ldots \ldots$

B.1 Jet Transfer Function parameters in RunIIb. . . . . . . . . . . . . . . . . . 99

C.1 Electron $p_{T}$ distribution for inclusive jet selection, $\geq 2$ jet selection, and final selection for the $e \mu$ channel. . . . . . . . . . . . . . . . . . 100

C.2 Electron $\eta_{\text {det }}$ distribution for inclusive jet selection, $\geq 2$ jet selection, and final selection for the $e \mu$ channel. . . . . . . . . . . . . . . . . . . . . . 100

C.3 Electron $\phi$ distribution for inclusive jet selection, $\geq 2$ jet selection, and final selection for the $e \mu$ channel. . . . . . . . . . . . . . . . . . . . . . . . 101

C.4 Muon $p_{T}$ distribution for inclusive jet selection, $\geq 2$ jet selection, and final selection for the $e \mu$ channel. . . . . . . . . . . . . . . . . . . . . . . 101

C.5 Muon $\eta_{\text {det }}$ distribution for inclusive jet selection, $\geq 2$ jet selection, and final selection for the $e \mu$ channel. . . . . . . . . . . . . . . . . . . . . 101

C.6 Muon $\phi$ distribution for inclusive jet selection, $\geq 2$ jet selection, and final selection for the $e \mu$ channel. . . . . . . . . . . . . . . . . . . . . . . . 101

C.7 Leading Jet $p_{T}$ distribution for inclusive jet selection, $\geq 2$ jet selection, and final selection for the $e \mu$ channel. . . . . . . . . . . . . . . . . . . . 102

C.8 Leading Jet $\eta_{\text {det }}$ distribution for inclusive jet selection, $\geq 2$ jet selection, and final selection for the $e \mu$ channel. . . . . . . . . . . . . . . . . . . . . . 102

C.9 Leading Jet $\phi$ distribution for inclusive jet selection, $\geq 2$ jet selection, and final selection for the $e \mu$ channel. . . . . . . . . . . . . . . . . . . . 102

C.10 Second Jet $p_{T}$ distribution for $\geq 2$ jet selection, and final selection for the $e \mu$ channel. . . . . . . . . . . . . . . . . . . 102

C.11 Second Jet $\eta_{\text {det }}$ distribution for $\geq 2$ jet selection, and final selection for the $e \mu$ channel. . . . . . . . . . . . . . . . . . . 103

C.12 Second Jet $\phi$ distribution for $\geq 2$ jet selection, and final selection for the $e \mu$ channel. . . . . . . . . . . . . . . . . . . . 103 
C.13 $\not \not_{T}$ distribution for inclusive jet selection, $\geq 2$ jet selection, and final selection for the $e \mu$ channel. . . . . . . . . . . . . . . . . . . . . . . 103

C.14 $m_{e m u}$ distribution for inclusive jet selection, $\geq 2$ jet selection, and final selection for the $e \mu$ channel. . . . . . . . . . . . . . . . . . . . . . . 103

C.15 $N_{\text {jets }}$ distribution for inclusive jet selection, $\geq 2$ jet selection, and final selection for the $e \mu$ channel. . . . . . . . . . . . . . . . . . . . . 104

C.16 Leading Electron $p_{T}$ distribution for inclusive jet selection, $\geq 2$ jet selection, and final selection for the ee channel. . . . . . . . . . . . . . . . 105

C.17 Leading Electron $\eta_{\text {det }}$ distribution for inclusive jet selection, $\geq 2$ jet selection, and final selection for the ee channel. . . . . . . . . . . . . . . 105

C.18 Leading Electron $\phi$ distribution for inclusive jet selection, $\geq 2$ jet selection, and final selection for the ee channel. . . . . . . . . . . . . . . . . . 105

C.19 Second Electron $p_{T}$ distribution for inclusive jet selection, $\geq 2$ jet selection, and final selection for the ee channel. . . . . . . . . . . . . . . 105

C.20 Second Electron $\eta_{\text {det }}$ distribution for inclusive jet selection, $\geq 2$ jet selection, and final selection for the ee channel. . . . . . . . . . . . . . . . . . 106

C.21 Second Electron $\phi$ distribution for inclusive jet selection, $\geq 2$ jet selection, and final selection for the ee channel. . . . . . . . . . . . . . . . . . 106

C.22 Leading Jet $p_{T}$ distribution for inclusive jet selection, $\geq 2$ jet selection, and final selection for the ee channel. . . . . . . . . . . . . . . . . . . 106

C.23 Leading Jet $\eta_{\text {det }}$ distribution for inclusive jet selection, $\geq 2$ jet selection, and final selection for the ee channel. . . . . . . . . . . . . . . . . . 106

C.24 Leading Jet $\phi$ distribution for inclusive jet selection, $\geq 2$ jet selection, and final selection for the ee channel. . . . . . . . . . . . . . . . . . . . 107

C.25 Second Jet $p_{T}$ distribution for $\geq 2$ jet selection, and final selection for the ee channel. . . . . . . . . . . . . . . . . . . . . 107

C.26 Second Jet $\eta_{\text {det }}$ distribution for $\geq 2$ jet selection, and final selection for the ee channel. 
C.27 Second Jet $\phi$ distribution for $\geq 2$ jet selection, and final selection for the ee channel. . . . . . . . . . . . . . . . . . . . 107

C. $28 \not \not T_{T}$ distribution for inclusive jet selection, $\geq 2$ jet selection, and final selection for the ee channel. . . . . . . . . . . . . . . . . . . . . . 108

C.29 $m_{e e}$ distribution for inclusive jet selection, $\geq 2$ jet selection, and final selection for the ee channel. . . . . . . . . . . . . . . . . . 108

C.30 $N_{\text {jets }}$ distribution for inclusive jet selection, $\geq 2$ jet selection, and final selection for the $e e$ channel. . . . . . . . . . . . . . . . . . . 108

C.31 Leading Muon $p_{T}$ distribution for inclusive jet selection, $\geq 2$ jet selection, and final selection for the $\mu \mu$ channel. . . . . . . . . . . . . . . . . . . 109

C.32 Leading Muon $\eta_{\text {det }}$ distribution for inclusive jet selection, $\geq 2$ jet selection, and final selection for the $\mu \mu$ channel. . . . . . . . . . . . . . . . 109

C.33 Leading Muon $\phi$ distribution for inclusive jet selection, $\geq 2$ jet selection, and final selection for the $\mu \mu$ channel. . . . . . . . . . . . . . . . 109

C.34 Second Muon $p_{T}$ distribution for inclusive jet selection, $\geq 2$ jet selection, and final selection for the $\mu \mu$ channel. . . . . . . . . . . . . . . . 109

C.35 Second Muon $\eta_{\text {det }}$ distribution for inclusive jet selection, $\geq 2$ jet selection, and final selection for the $\mu \mu$ channel. . . . . . . . . . . . . . . . . . 110

C.36 Second Muon $\phi$ distribution for inclusive jet selection, $\geq 2$ jet selection, and final selection for the $\mu \mu$ channel. . . . . . . . . . . . . . . . . . . . 110

C.37 Leading Jet $p_{T}$ distribution for inclusive jet selection, $\geq 2$ jet selection, and final selection for the $\mu \mu$ channel. . . . . . . . . . . . . . . . . . 110

C.38 Leading Jet $\eta_{\text {det }}$ distribution for inclusive jet selection, $\geq 2$ jet selection, and final selection for the $\mu \mu$ channel. . . . . . . . . . . . . . . . . . . 110

C.39 Leading Jet $\phi$ distribution for inclusive jet selection, $\geq 2$ jet selection, and final selection for the $\mu \mu$ channel. . . . . . . . . . . . . . . . . . . 11

C.40 Second Jet $p_{T}$ distribution for $\geq 2$ jet selection, and final selection for the $\mu \mu$ channel. . . . . . . . . . . . . . . . . . . 111 
C.41 Second Jet $\eta_{\text {det }}$ distribution for $\geq 2$ jet selection, and final selection for the $\mu \mu$ channel. . . . . . . . . . . . . . . . . . . . 111

C.42 Second Jet $\phi$ distribution for $\geq 2$ jet selection, and final selection for the $\mu \mu$ channel. . . . . . . . . . . . . . . . . . . . . . . 111

C.43 $\not p_{T}$ distribution for inclusive jet selection, $\geq 2$ jet selection, and final selection for the $\mu \mu$ channel. . . . . . . . . . . . . . . . . . . . . . 112

C.44 $m_{\mu \mu}$ distribution for inclusive jet selection, $\geq 2$ jet selection, and final selection for the $\mu \mu$ channel. . . . . . . . . . . . . . . . . . . . . . 112

C.45 $\not_{T}^{\sigma}$ distribution for inclusive jet selection, $\geq 2$ jet selection, and final selection for the $\mu \mu$ channel. . . . . . . . . . . . . . . . . . . . . . 112

C.46 $p_{T}^{\sigma}$ distribution for inclusive jet selection, and $\geq 2$ jet selection, in Log scale. 112 C.47 $N_{\text {jets }}$ distribution for inclusive jet selection, $\geq 2$ jet selection, and final selection for the $\mu \mu$ channel. . . . . . . . . . . . . . . . . . 113 


\section{List of Abbreviations}

$X_{0} \quad \ldots \ldots \ldots \ldots \ldots \ldots$ Radiation Length

$\eta \quad \ldots \ldots \ldots \ldots$ Pseudorapidity: $\tanh ^{-1} \cos \theta \ldots \ldots$

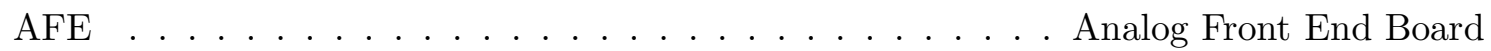

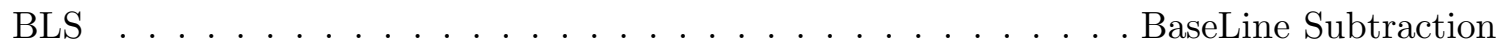

BLUE . . . . . . . . . . . . . . Best Linear Unbiased Estimator

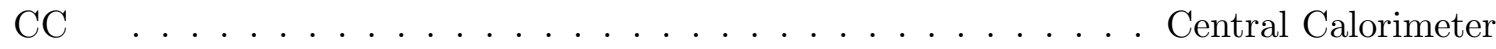

$\mathrm{CDF} \ldots \ldots \ldots \ldots$ Collider Detector a F . . . . . . . . . . . . . .

CFT ...................................... Fiber Tracker

CPS ................................. Pentral Preshower Detector

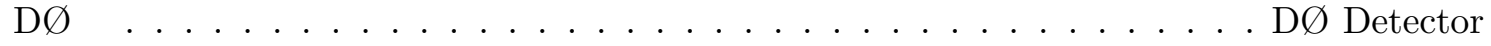

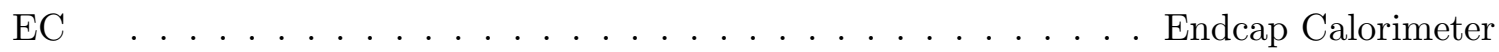

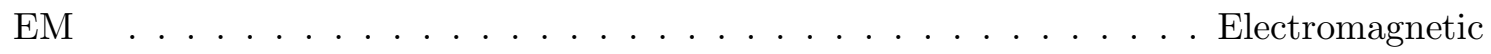

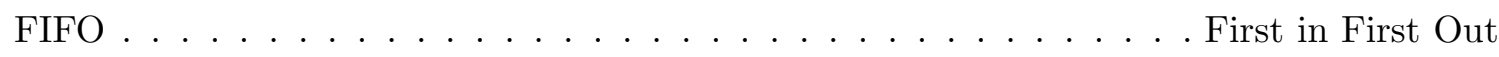

FPS ............................ Forward PreShower Detector

$\mathrm{GeV} \ldots \ldots \ldots \ldots$. . . . . . . . . . . . . . Electron Volt

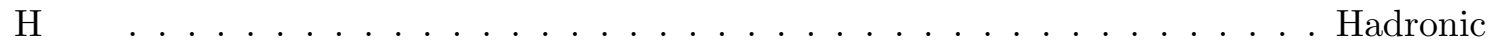

$\operatorname{JES} \ldots \ldots \ldots \ldots \ldots \ldots$ Jet Energy Scale

$\mathrm{L} 1 \ldots \ldots \ldots \ldots \ldots \ldots$ Level 1 Trigger

L1CAL . . . . . . . . . . . . . . . Level 1 Calorimeter Trigger 
L1CalTrk . . . . . . . . . . . . Level 1 Calorimeter Track Trigger L1CTT . . . . . . . . . . . . . . Level 1 Central Track Trigger L1PS . . . . . . . . . . . . . . . Level 1 PreShower Trigger

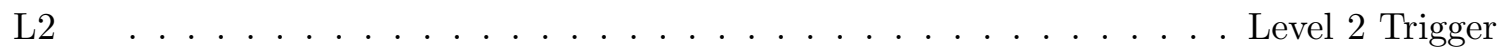

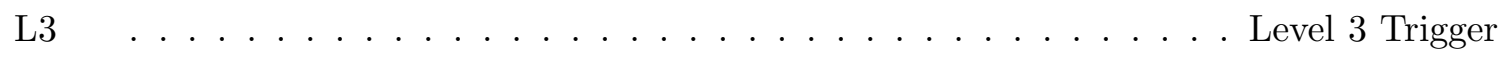

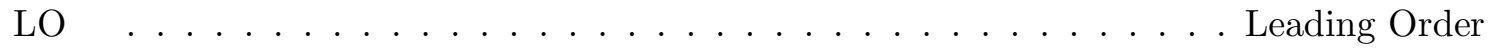
$\mathrm{MeV} \ldots \ldots \ldots \ldots \ldots$ Mega Electron Volt

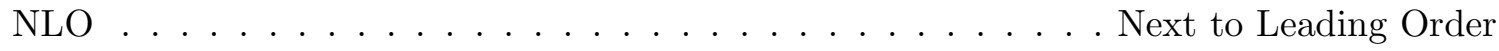
NNLL . . . . . . . . . . . . . . . . Next-to-Next-to-Leading-Logarithm NNLO . . . . . . . . . . . . . . . Next-to-Next-to-Leading-Order QCD ...................................... ChromoDynamics $\mathrm{SBC} \ldots \ldots \ldots \ldots . \ldots \ldots$ Single Board Computer

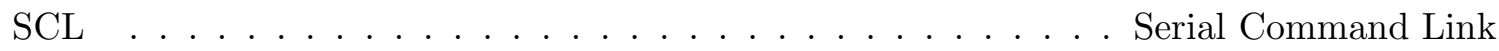

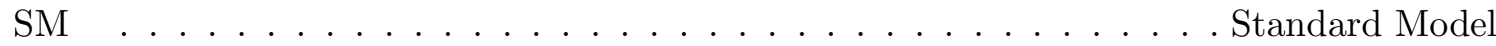
$\mathrm{SMT} \ldots \ldots \ldots . \ldots . \ldots . . \ldots$ Silicon Microstrip Tracker $\mathrm{TeV} \ldots \ldots \ldots \ldots \ldots \ldots \ldots$ Tera Electron Volt VLPC . . . . . . . . . . . . . . Visible Light Photon Counter 


\section{Chapter 1}

\section{Introduction}

\subsection{The Standard Model and the Top Quark}

There are four fundamental forces of nature, gravity, electromagnetism, the weak nuclear force, and the strong nuclear force. The Standard Model (SM) is the theory of three of these forces (EM,weak, and strong), and the particles on which these forces act.

The strong force is described by QCD (Quantum ChromoDynamics), a quantum field theory with an $\mathrm{SU}(3)_{c}$ gauge group, and an asymptotically free coupling. Asymptotic freedom means that the strong force is weak at high energies or small distances, and only becomes strong at low energies or large distances. The strong force becomes strong enough at large distances that when colored quarks and gluons become separated, the binding energy between them is large enough to create new quarks and gluons. All the free quarks and gluons ultimately become bound into hadrons. This process of hadronization has a characteristic scale of $\Lambda_{Q C D} \approx 200 \mathrm{MeV}$, which corresponds to a time scale of $\tau=4 \times 10^{-24}$ S.

The electromagnetic and weak forces are combined together into a single electroweak force which obeys an $\mathrm{SU}(2)_{L} \times \mathrm{U}(1)_{Y}$ gauge group. The $\mathrm{SU}(2)_{L}$ group has three generators and the $\mathrm{U}(1)_{Y}$ group has one, corresponding to four gauge bosons that mediate the electroweak force: $W_{\mu}^{1}, W_{\mu}^{2}, W_{\mu}^{3}$ for $\mathrm{SU}(2)_{L}$ and $B_{\mu}$ for $\mathrm{U}(1)_{Y}$. The $\mathrm{SU}(2)_{L} \times \mathrm{U}(1)_{Y}$ symmetry of the electroweak interaction is spontaneously broken through its coupling to the scalar 
higgs field. The higgs field has a degenerate ground state at a finite value of the field and thus spontaneously breaks the $\mathrm{SU}(2)_{L} \times \mathrm{U}(1)_{Y}$ symmetry. The four massless gauge bosons before symmetry breaking turn into three massive gauge bosons $\left(W^{+}, W^{-}, Z^{0}\right)$, and the massless photon which obeys an unbroken $\mathrm{U}(1)_{E M}$ gauge symmetry.

The SM contains two different types of fermions, quarks and leptons. The quarks have non-zero $\mathrm{SU}(3)_{c}$ "color" charges, and come in electroweak doublets of up-type quarks with charge $+2 / 3$ and down-type quarks with charge $-1 / 3$. There are three generations of quarks, each with its own electroweak doublet:

$$
\left(\begin{array}{l}
u \\
d
\end{array}\right)_{L},\left(\begin{array}{l}
c \\
s
\end{array}\right)_{L},\left(\begin{array}{l}
t \\
b
\end{array}\right)_{L}
$$

The other type of fermions in the standard model are the charged leptons and the neutral neutrinos, which form electroweak doublets in three generations:

$$
\left(\begin{array}{c}
\nu_{e} \\
e^{-}
\end{array}\right)_{L},\left(\begin{array}{c}
\nu_{\mu} \\
\mu^{-}
\end{array}\right)_{L},\left(\begin{array}{c}
\nu_{\tau} \\
\tau^{-}
\end{array}\right)_{L}
$$

The SM is a chiral theory, fermions come in left-handed and right-handed varieties, and the weak force couples to left-handed fermions and right-handed anti-fermions. While the charged leptons come in both left-handed and right-handed forms, which mix due the mass of the charged leptons, the neutrinos couple exclusively to the weak force, so that only left-handed neutrinos (or right-handed anti-neutrinos) can be directly observed. To our previous list of quarks we must add:

$$
u_{R}, d_{R}, c_{R}, t_{R}, b_{R}
$$

While to our list of leptons we must add:

$$
e_{R}^{-}, \mu_{R}^{-}, \tau_{R}^{-}
$$

In the simplest version of the standard model, neutrinos are massless, and the flavor of a neutrino is conserved. However, the oscillation of neutrino flavor has been experimentally observed, which implies that neutrinos have mass. Massive neutrinos require a modification 
of the SM through the addition of either right-handed neutrinos, or a more exotic Majorana mass term (where neutrinos are their own antiparticle).

The masses of the fermions in the standard model increase from one generation to the next, but their values are otherwise arbitrary. The most massive of the fermions is the top quark, and with $m_{t}=173 \mathrm{GeV}$, the top quark mass is 40 times larger than the mass of the next most massive quark. The top mass has a lifetime of $\sim 0.5 \times 10^{-24} \mathrm{~s}$, corresponding to a decay width of $1.5 \mathrm{GeV}$. This is quite a bit larger than $\Lambda_{Q C D} \approx 200 \mathrm{MeV}$. As a result, the top quark decays before it has a chance to hadronize. Because of this, we can directly reconstruct the four momenta of the top from its decay products, and measure the charge, spin, and helicity of the top.

\subsection{Top Quark Mass, Constraint on Higgs Mass}

As the most massive fermion in the standard model, the top plays a special role in radiative corrections to the mass of the $\mathrm{W}$, the top makes a contribution to one-loop level of $\Delta m_{W} \propto m_{t}^{2}$, while the higgs boson makes a contribution $\Delta m_{W} \propto \log m_{h}$. These radiative corrections to $m_{W}$ are well understood, and a measurement of $m_{W}$ and $m_{t}$, along with other precision measurements $\left(m_{Z}, \Gamma_{Z}, \tau_{\mu}\right.$, etc) can be turned into a constraint on $m_{\text {higgs }}$. The potential to constrain the mass of the as yet undiscovered higgs boson is a powerful motivation for precisely measuring both $m_{W}$ and $m_{t}$. Figure 1.1 shows the most precise current constraint on $m_{H}$.

If we consider extensions to the Standard Model, $m_{t}$ remains an important input to any theory we might consider, and a precise measurement of $m_{t}$ can help us determine whether a particular model is capable of reproducing nature.

\subsection{Top Quark Pair Production and Decay}

To measure the top quark mass, we need to produce top quarks. The dominant production modes for top production at the Tevatron are single top production, and top pair produc- 


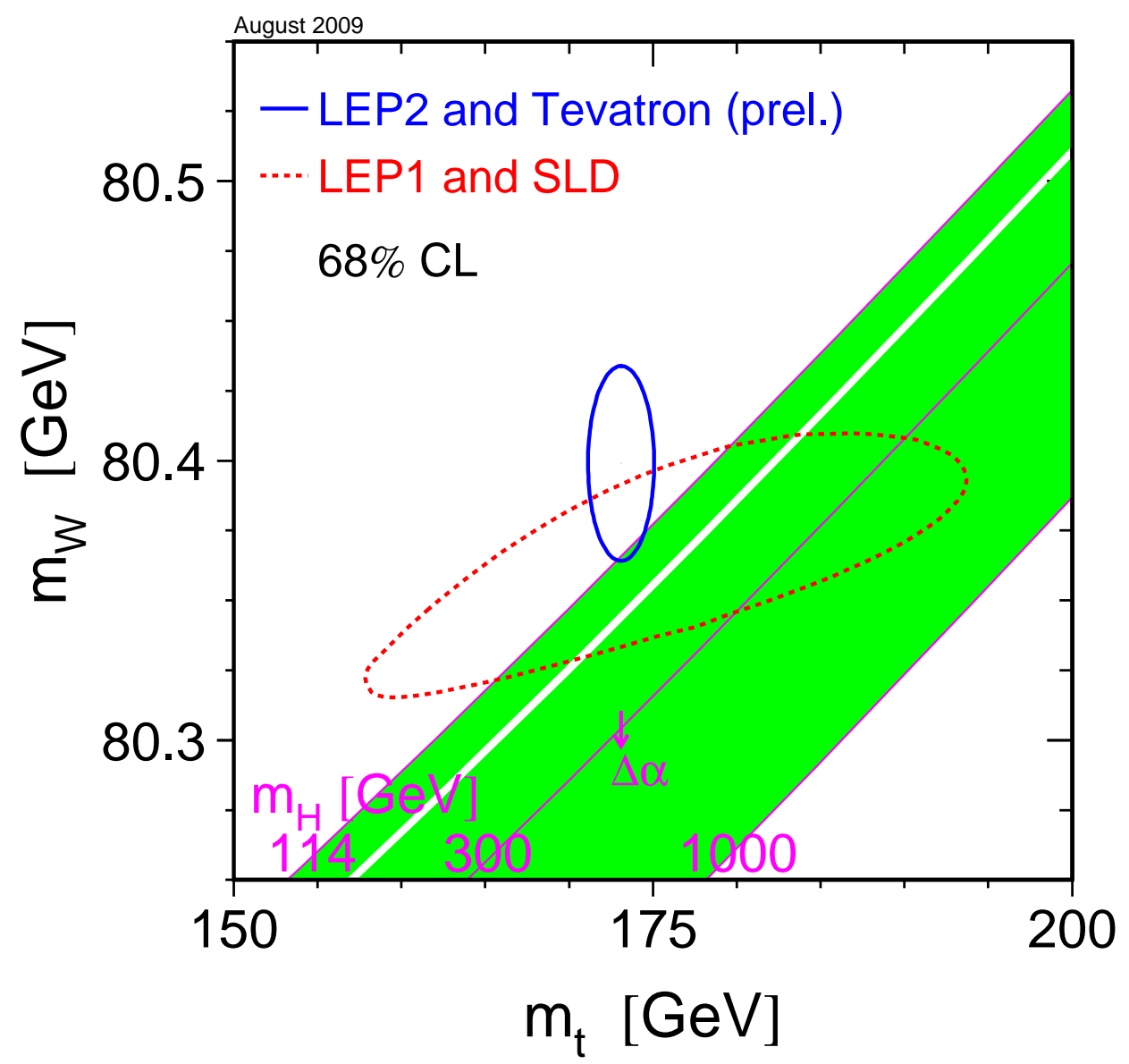

Figure 1.1: The larger dashed red band shows the $68 \%$ confidence limit (CL) for $m_{W}$ vs. $m_{t}$ determined using data from the first run of LEP and SLD, while the blue line shows the same for the second run of LEP and the Tevatron, the green band shows corresponding values of $m_{H}$ between 113 and $1000 \mathrm{GeV}$ [2]. 
tion. Single top production has a smaller cross section, but more significant is the similar topology of single top and the background processes $W+b \bar{b}$ and $W+2 j e t$ which have much higher cross sections. While top pair production was observed in Run I with $\sim 100 \mathrm{pb}^{-1}$, single top production has only recently been observed in Run II with $>1 \mathrm{fb}^{-1}$.

Each top quark first decays into a $b$ quark, and a $W$ boson. The $W$ boson then decays into either a pair of quarks, or a charged lepton and neutrino. Each produced quark undergoes fragmentation and hadronization, producing a jet. The final state of top pair production can be classified according to the decay of the $W$ : both $W$ decaying to quarks: the all-jets channel, one $W$ decaying to leptons: the lepton+jets channel, and both $W$ 's decaying to leptons: the dilepton channel (see Fig. 1.2).

This thesis will focus exclusively on those final states with two reconstructed leptons, either: two $e$ 's, two $\mu$ 's, an $e$ and a $\mu$, an $e$ (or $\mu$ ) and a charged isolated track (which is assumed to come from an $e$ or $\mu$ ). This will mostly involve direct $W$ decays $(W \rightarrow e, W \rightarrow$ $\mu)$, but will include cascade decays involving a $\tau$ lepton $\left(W \rightarrow \tau \nu_{\tau} \rightarrow e \nu_{e} \nu_{\tau}\right)$.

The Standard Model naturally predicts that the top mass is independent of the decay mode of the top, and any variation of the measured top mass between channels would be an indication of non-SM behavior. Such a difference could indicate the existence of some exotic process mimicking the signature of one channel of SM $t \bar{t}$ but not the others.

\subsubsection{Theoretical Predictions of Production Cross Section}

The total cross section has the form:

$$
\sigma\left(s, m_{t}, \mu_{r}, \mu_{f}\right)=\sum_{i, j=g, q, \bar{q}} f_{i}\left(\mu_{f}^{2}\right) \otimes f_{j}\left(\mu_{f}^{2}\right) \otimes \hat{\sigma}\left(m_{t}, \mu_{r}, \mu_{f}\right)[14] .
$$

Where $s$ is the square of the center-of-mass energy of the proton anti-proton system, $f_{i}$ are the parton distribution functions (describing the distribution of initial state partons), $\hat{\sigma}$ is the parton level cross section, and $\otimes$ stands for the convolution over the allowed parton momenta.

Theoretical calculations of the cross section have been performed to various orders in 


\section{Top Pair Decay Channels}

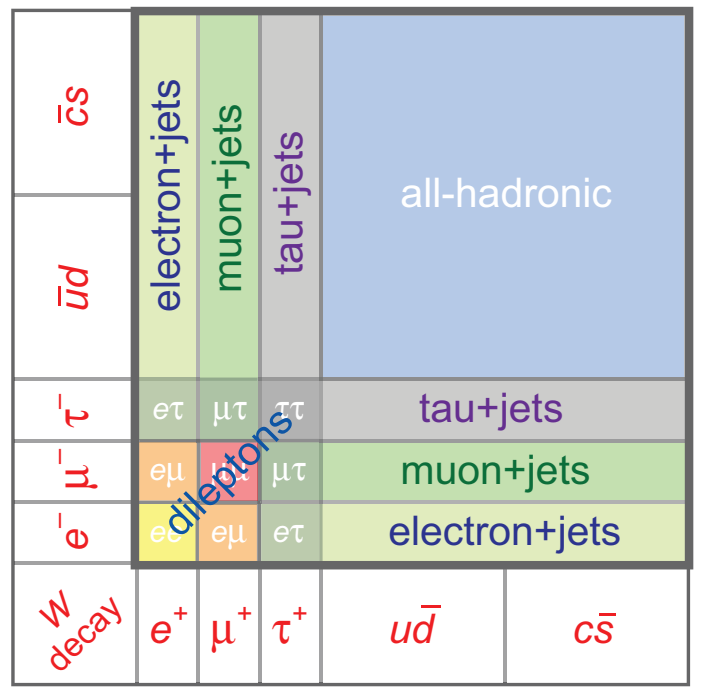

Figure 1.2: Decay Channels for Top Quark Pair Production.

perturbation theory. The best results so far are found using a perturbation theory calculation performed to Next-to-Next-to-Leading-Order (NNLO) in the strong coupling constant $\alpha_{S}$, with the resummation of logarithmic terms due to soft gluon emission performed to Next-to-Next-to-Leading-Logarithm (NNLL). This calculation gives a theoretical cross section of:

$$
\sigma\left(s=\sqrt{1.96 \mathrm{TeV}}, m_{t}=172.5 \mathrm{GeV}\right)=7.46_{-0.67}^{+0.48} \mathrm{pb}[14] .
$$

Figure 1.3 compares the NLO prediction with both the NNLO, and NLO+NLL(next to leading $\log )$.

\subsubsection{Measurement of Top Mass using Measured Cross Section}

The production cross section can be measured in data by looking at the expected yield:

$$
N_{\text {exp }}\left(\sigma_{t \bar{t}}\right)=\mathcal{L} \sigma_{t \bar{t}} \operatorname{Br}(t \bar{t} \rightarrow X) \epsilon_{t \bar{t} \rightarrow X}+N_{b k g} .
$$

Where $\mathcal{L}$ is the integrated luminosity, $\operatorname{Br}(t \bar{t} \rightarrow X)$ is the branching fraction for $t \bar{t}$ 
into the decay channel being looked at, and $\epsilon_{t \bar{t} \rightarrow X}$ is the selection efficiency. A Poisson likelihood is then used to extract the cross section from the observed yield in data. DØ has measured the cross section for top pair production in this way in the lepton+jet, dilepton, and lepton $+\tau$ channels with $1 \mathrm{fb}^{-1}$, and measures a cross section of:

$$
\sigma_{t \bar{t}}=8.18_{-0.86}^{+0.98} \mathrm{pb}[15]
$$

For $m_{t}=170 \mathrm{GeV}$. By repeating this procedure at different top mass values, the mass dependence of the measured $\sigma_{t \bar{t}}$ is obtained, and comparing $\sigma_{t \bar{t}}^{\text {measured }}\left(m_{t}\right)$ to $\sigma_{t \bar{t}}^{t h e o r y}\left(m_{t}\right)$, it is possible to measure the top mass indirectly from the measured cross section, D $\varnothing$ has done this and obtains:

$$
m_{t}=169.1_{-5.2}^{+5.9} \mathrm{GeV}[15]
$$

It should be mentioned that this indirect measurement is dependent on the order in perturbation theory used in the theoretical prediction, a prediction to leading order (LO) produces a different, smaller, top mass than a prediction to next-to-leading order (NLO).

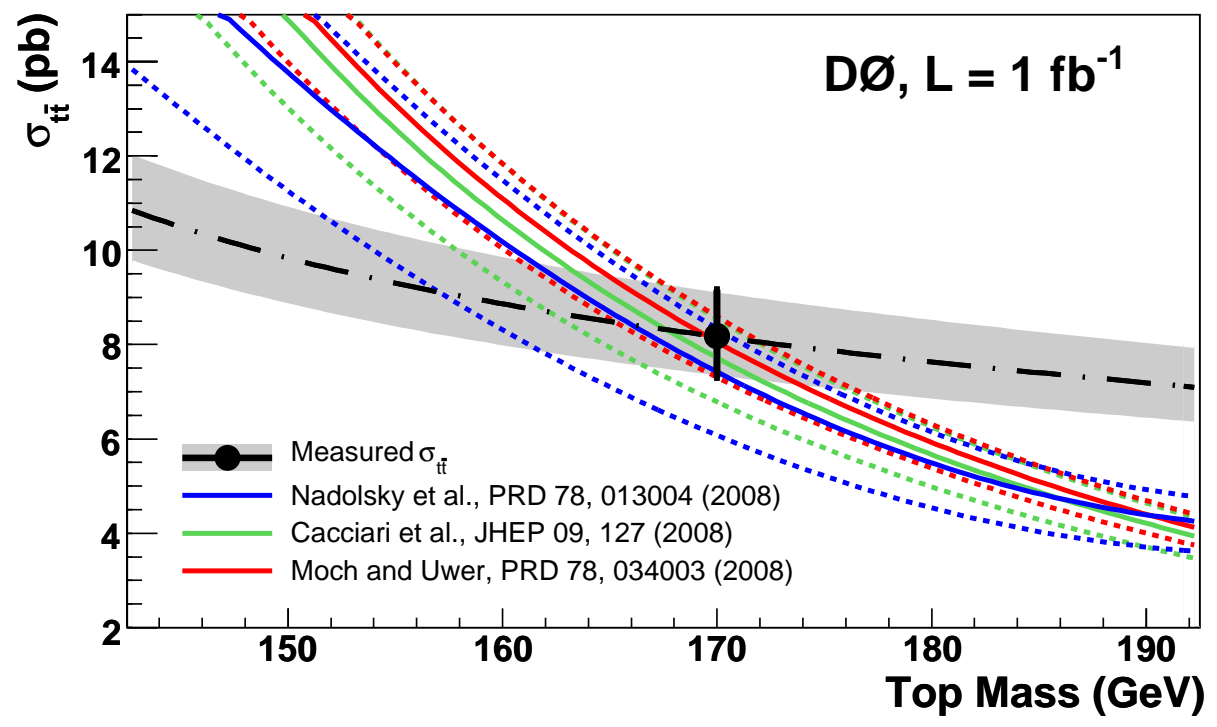

Figure 1.3: Theory cross section vs. measured cross section [3], the grey band gives the error on the measured cross section. 


\subsection{Tevatron}

The Tevatron is a $p \bar{p}$ collider with a center of mass energy of $1.96 \mathrm{TeV}$. A Cockroft-Walton generator is used to produce $H^{-}$ions and accelerate them to $750 \mathrm{keV}$. The ions are then accelerated in the Linac, or linear accelerator, to an energy of $400 \mathrm{MeV}$. A carbon foil is then used to remove the electrons from the $H^{-}$ions. The resulting protons are then sent into the Booster, a cyclotron accelerator which accelerates the protons to $8 \mathrm{GeV}$.

From the Booster, the protons are sent into the Main Injector, a ring which further accelerates the protons. Protons from the Main Injector, at an energy of $120 \mathrm{GeV}$ are sent into a carbon target, producing a small number of anti-protons (100,000 protons are required for each anti-proton). The antiprotons are collected and cooled in the accumulator. They are stored in the accumulator for a short time, before being transferred into the Main Injector, and then into the Recycler, where they are cooled and stored over a much longer period (around 12 hours).

The running of the Tevatron is broken up into stores. Each store begins by injecting protons and anti-protons from the Main Injector at an energy of $150 \mathrm{GeV}$. After the $p$ 's and $\bar{p}$ 's have been injected, they are both accelerated to a beam energy of $980 \mathrm{GeV}$. The $p$ 's and $\bar{p}$ 's travel in separate helical orbits around the Main Ring, and quadrupole magnets are used at the interaction regions to focus the beams to a fixed point at the center of the $\mathrm{D} \varnothing$ and CDF detectors. 

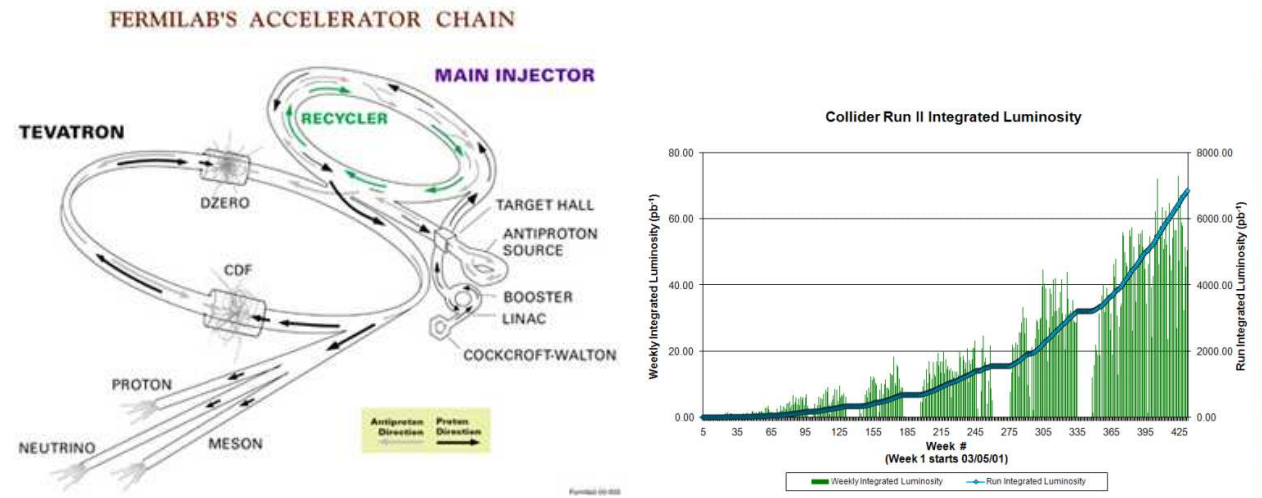

Figure 1.4: Diagram of Tevatron Accelerator Chain(left), Integrated Luminosity as a Function of Time at the Tevatron(right). 


\section{Chapter 2}

\section{Detector}

The $\mathrm{D} \varnothing$ detector is made up of three main detector components, a central tracker, a sampling calorimeter, and a muon detector. The central tracker sits directly on top of the beam line, and is entirely inside of a solenoid magnet with a magnetic field of $\sim 2$ T. The calorimeter surrounds the solenoid, and the muon system lies just outside of the calorimeter and surrounds the toroidal magnets[4], see Fig 2.1.

The center of the $\mathrm{D} \varnothing$ coordinate system is the center of the detector. The z-axis lies along the beamline, positive z points south in the director of the proton beam, negative $\mathrm{z}$ points north in the direction of the anti-proton beam.

When proton anti-proton collisions are taking place, the detector is being bombarded by large numbers of electrons, photons, muons, and hadrons. These particles interact with the detector, producing signals which are transfered out of the detector electronically. The collider produces approximately two million collisions each second. To deal with this overwhelming amount of data, a reduced sample of the signals from the detectors is used by the online trigger system to quickly decide whether to accept or reject a given event.

\subsection{Tracker}

The tracking detector is used to measure the trajectory of charged particles emerging from the interaction point, as they travel through the central magnetic field. There are two 


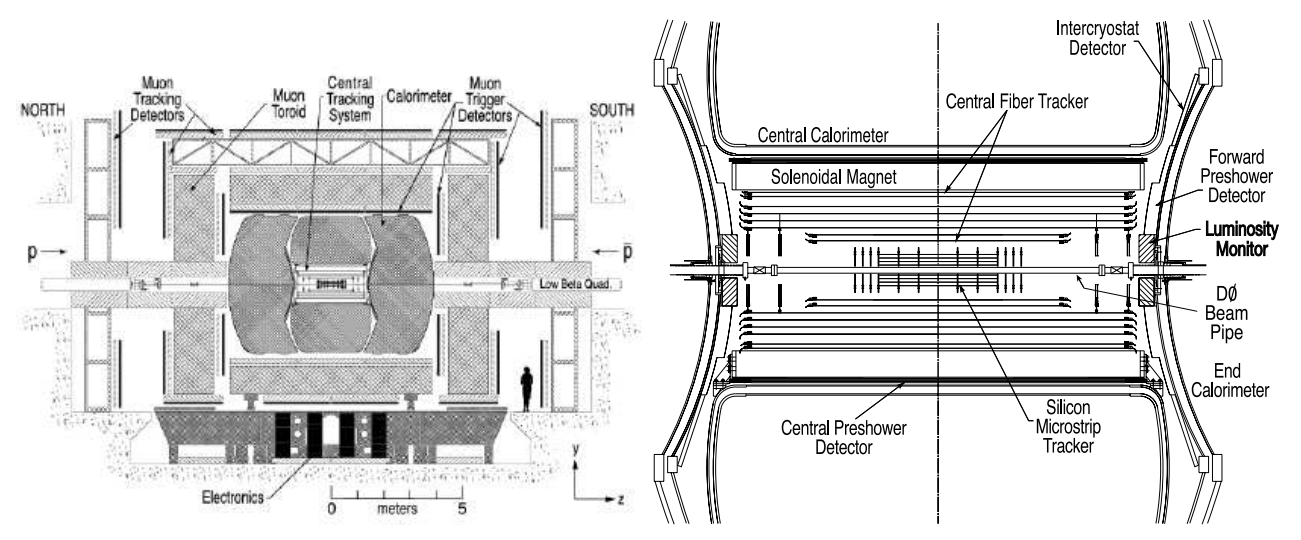

Figure 2.1: Diagram of current DØdetector (left) and central detector (right) [4]

parts, the Silicon Microstrip Tracker (SMT), which sits just outside the beampipe, and the Central Fiber Tracker (CFT) which surrounds the SMT.

\subsubsection{Silicon Microstrip Tracker}

The SMT uses silicon semiconductor as an active medium to detect the passage of charged particles. It outputs information about the position of hits associated with tracks, this information is combined with hit information from the CFT to reconstruct the tracks left behind by charged particles. The SMT sits close to the interaction region, and can resolve hits to $\sim 20 \mu \mathrm{m}$. This allows the SMT to resolve the impact parameter of tracks (distance of closest approach to the primary interaction point), and the position of displaced secondary vertices with a precision approaching $\sim 20 \mu \mathrm{m}$. These high precision measurements are important as both large impact parameters and well displaced secondary verticies are signals of the presence of B-hadrons. B-hadrons are produced from hadronized b-quarks, and have a relatively long lifetime corresponding to a decay length of $500 \mu \mathrm{m}$.

\section{Sensors}

Silicon sensors come in various forms, but all use the same physics. Charged particles passing through a lightly doped n-type semiconductor excite loosely bound electrons, producing an electron-hole pair. The semiconductor has an electric field applied to it and the electron and hole are pulled to opposite ends of the semiconductor, where they interact 
with highly doped strips of p-type or n-type semiconductor. These strips are connected to the readout electronics, and transmit the electrical signal out of the detector.

\section{Detector Layout}

During DØ Run IIa, the SMT was made up of 6 barrels, running parallel to the beam, 12 F-disks, interspersed between the barrels and $4 \mathrm{H}$-disks at high $|z|$ (see figure 2.2). For DØ Run IIb, an extra layer of barrel detectors were added between the innermost barrel and the beampipe, further improving the precision of the SMT detector.

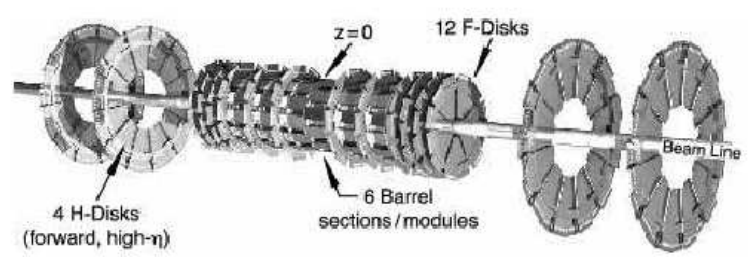

Figure 2.2: SMT detector [4].

\subsubsection{Central Fiber Tracker}

The Central Fiber Tracker (CFT) sits in between the toroid magnet and the SMT. It uses scintillating fibers to measure the position of charged particles, and ultimately measure their momentum. The scintillating fibers produce signals fast enough to be used in the first level of the $\mathrm{D} \varnothing$ trigger system.

\section{Layout}

The CFT is composed of 8 concentric cylinders, filling the space from 20 to $50 \mathrm{~cm}$ from the beam. The 2 innermost cylinders are $1.66 \mathrm{~m}$ long, while the 6 outer cylinders are 2.52 m long, . This gives the CFT coverage of $|\eta| \lesssim 1.7$, where $\eta$ is the pseudorapidity defined as: $\eta=\tanh ^{-1} \cos \theta$.

Each cylinder contains one axial doublet, where each doublet has two layers of fibers see Fig. 2.3, and one stereo doublet where the stereo angle alternates between $+3^{\circ}$ and 
$-3^{\circ}$. The axial layers are used for the level 1 track trigger (L1CTT).

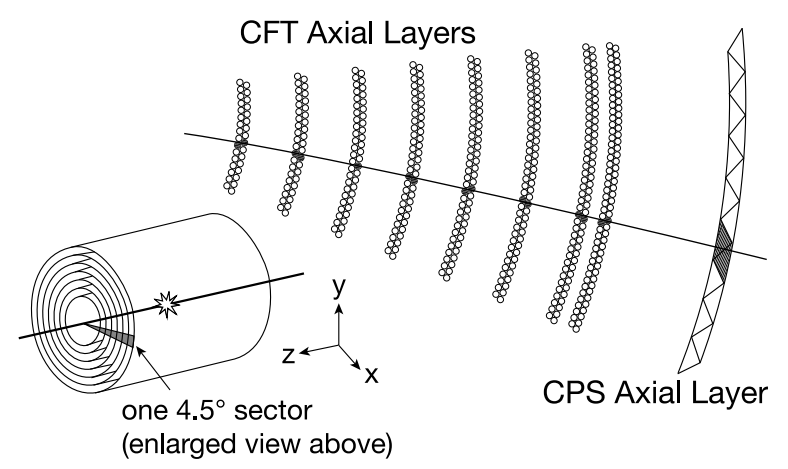

Figure 2.3: Layout of CFT and CPS axial fibers [4].

\section{Fibers}

The fibers of the CFT are mostly clear Polystyrene plastic. This cheap material absorbs energy from passing charged particles, and doubles as a light transmitter. The Polystyrene is doped with Paraterphenyl at $1 \%$ by weight. Charged particles passing through the fibers excite electrons in the Polystyrene, and these excitations are transferred to the Paraterphenyl via a non-radiative dipole interaction. Once excited, the Paraterphenyl undergoes a rapid fluorescent decay ( $\approx 1 \mathrm{~ns}$ ), emitting light at a wavelength of $340 \mathrm{~nm}$. This light doesn't transfer well through Polystyrene, so 3-hydroxyflavone (3HF) is added to the fibers at $1500 \mathrm{ppm}$, the $3 \mathrm{HF}$ absorbs the 340nm light, and remits light at 530nm (giving the CFT fiber a distinct green color). This combination of Polystyrene, Paraterphenyl and $3 \mathrm{HF}$ is referred to as a wavelength shifting fiber (WLS).

The fibers of the CFT are mostly clear Polystyrene plastic. The Polystyrene is doped with Paraterphenyl at $1 \%$ by weight. Charged particles passing through the fibers excite electrons in the Polystyrene, and these excitations are transferred to the Paraterphenyl via a non-radiative dipole interaction. Once excited, the Paraterphenyl undergoes a rapid fluorescent decay $(\approx 1 \mathrm{~ns}$ ), emitting light at a wavelength of $340 \mathrm{~nm}$. This light doesn't transfer well through Polystyrene, so 3-hydroxyflavone (3HF) is added to the fibers at 1500 ppm, the $3 \mathrm{HF}$ absorbs the $340 \mathrm{~nm}$ light, and remits light at 530nm (giving the CFT fiber 
a distinct green color).

\section{Readout}

The scintillating fibers are coupled to clear fiber Polystyrene waveguides, which transfer the light to the visible light photon counters (VLPC's) which convert the photons' visible light signals into analog electrical signals.

The VLPC's use impurity band silicon avalanche photodetectors which operate at a temperature of 9K. They are arranged into cassettes, with 128 VLPC chips per cassette. Each chip has 8 pixels, and each pixel is coupled to a single fiber from the detector. The bottom of each cassette sits in a cryostat, to keep the VLPC chips at 9K. The top of the cassette sits outside of the cryostat, and contains preamplifiers and analog front end (AFE) electronics boards. The AFE's perform a number of different functions. They provide signals for the L1 and L2 triggers, and readout for the L3 trigger, and are capable of detecting very small signals from the VLPC's. The VLPC's and AFE's are shared between the CFT and Preshower detectors.

\subsection{Solenoid Magnet}

The solenoid magnet provides a uniform axial $2 \mathrm{~T}$ magnetic field for the central tracking detectors, see Fig. 2.4. It sits just inside the central calorimeter cryostat, is $2.73 \mathrm{~m}$ long and has an inner diameter of $1.42 \mathrm{~m}$. The magnet is $\sim 1 X_{0}$ (radiation length) thick at normal incidence or $|\eta| \sim 0$.

\subsection{Central and Forward Preshower}

The Preshower detectors are intended to aid in the identification of electrons, and the rejection of backgrounds to electrons, both for triggering and for offline reconstruction. They provide a fast measurement of both the energy and momentum of particles, and complement both the tracking detectors and the calorimeter. The Central Preshower 


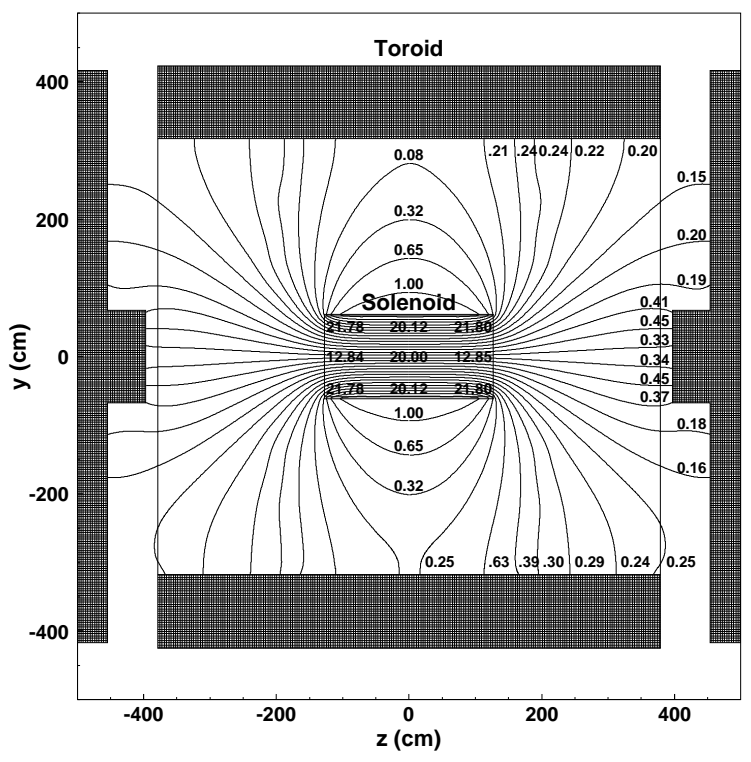

Figure 2.4: View of the DØ magnetic field showing both the solenoid and toroid magnets at full current [4].

(CPS) Detector provides a coverage of $|\eta|<1.3$, while the forward preshower (FPS) covers $1.5<|\eta|<2.5$.

Both the central and forward preshowers use triangular strips of scintillator, arranged so that there is no dead space between strips. The strips are made of Polystyrene, doped with paraterphenyl at $1 \%$ by weight and $150 \mathrm{ppm}$ of diphenyl stilbene. Each strip is wrapped in aluminized mylar, and at the center of each strip is a wavelength shifting (WLS) fiber which carries the signal out of the detector.

\subsection{Calorimeter}

The Calorimeter system is composed of 3 sampling liquid Argon (lAr) calorimeters: the central calorimeter (CC), north and south endcap calorimeters (ECN and ECS), and the intercryostat detector, which uses scintillation counters to sample particle showers between the croystats.

The calorimeters were designed to measure the energy of electrons, photons and jets in the absence of a central magnetic field. They also assist in the identification of electrons, 
photons and jets, and measure the missing transverse momentum $\left(\not p_{T}\right)$.

The CC provides coverage of $|\eta| \lesssim 1$. while the EC calorimeters extend the coverage of the detector to $|\eta| \approx 4$. Each calorimeter has its own cryostat and is maintained at a temperature of $90 \mathrm{~K}$.

Each calorimeter is divided into three transverse sections: an electromagnetic section (EM), which sits closest to the beam, and is optimized to detect electromagnetic showers, a fine hadronic section (fHad) designed to precisely measure the energy from hadronic showers, and the coarse hadronic section (cHad) which is furthest from the beam, and seeks to measure any energy from showers not absorbed by the previous two sections.

The sampling calorimeters are made up of metal absorbing plates, with liquid argon (lAr) as the active medium. The plates initiate showers, and absorb energy from them, while the lAr measures the energy deposited by charged particles passing through it.

A single unit of the calorimeter (see figure 2.5) is composed of alternating absorber plates and signal boards. An electric field is established by grounding the absorber plates and connecting the resistive surfaces of signal boards to positive high voltage $(2 \mathrm{kV})$. Charged particles knock electrons from the liquid argon, and these electrons drift to the absorber plates in less than approximately $450 \mathrm{~ns}$.

The width of the readout cells is matched to the typical width of an electromagnetic or hadronic shower. In the EM section the cells have a width of 1-2cm, while in the fHad and cHad the width is $10 \mathrm{~cm}$. The cells are further grouped into pseudo-projective towers with a size of $\eta \times \phi=0.1 \times 0.1$ (see fig. 2.5). Pseudo-projective means that the boundaries of the towers only approximately lie along lines of constant $\eta$.

The calorimeter has 55,296 available channels for readout, of which 47,032 are physically connected to the detector. The signals in the readout from the detector are first sent to charge preamplifiers just outside the cryostat via low impedance coaxial cables. From there they travel over twisted-pair cables to the baseline subtractor (BLS) boards.

The BLS boards perform several different functions. They store the raw signals from the calorimeter using Switched Capacitor Arrays (SCA's) as analog storage devices, for 
the $4 \mu \mathrm{s}$ required for a L1 trigger decision to be made, and the additional $2 \mathrm{~ms}$ required for a L2 trigger decision. They do baseline subtraction to remove low frequency noise or pileup from the signal, and they provide inputs to the L1 and L2 calorimeter trigger. Pileup refers to a signal from one beam crossing which affects the calorimeter signal during a later beam crossing, this is an issue as the 132 ns between beam crossings is less than the $450 \mathrm{~ns}$ required for a signal in a calorimeter cell to die away.
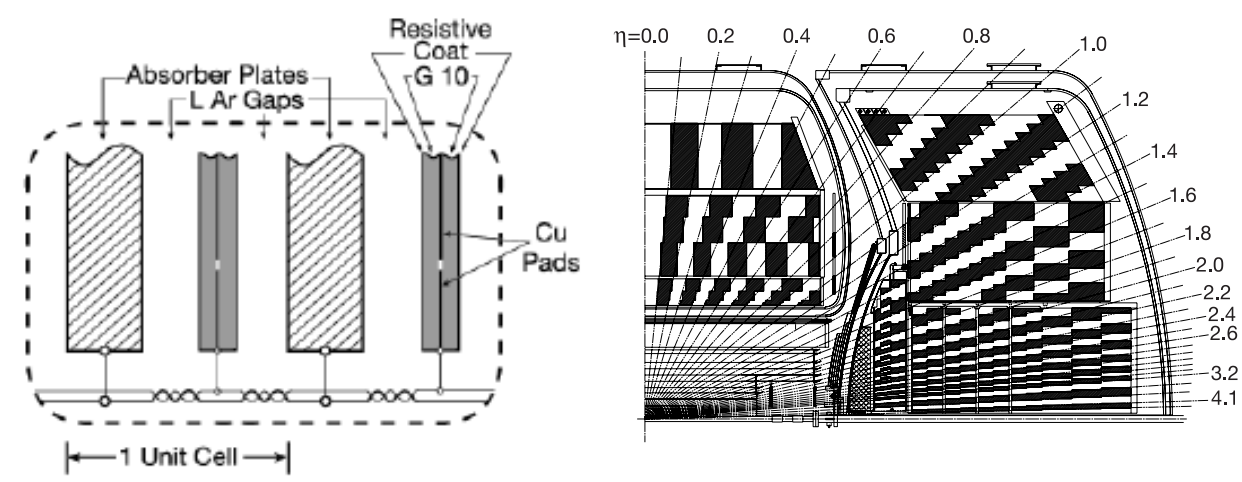

Figure 2.5: Diagram of calorimeter cell(left), Charged particle traveling through solenoid magnetic field(right) [4].

\subsubsection{Inter Cryostat Detector}

The calorimeter offers only incomplete coverage in the region $0.8<|\eta|<1$.4, to compensate for this extra detectors have been added. The first of these are massless-gaps, which are readout cells in front of the first layer of uranium absorber. The second of these is the Inter Cryostat Detector (ICD).

The ICD is made up of scintillator on the interior surfaces of the end cryostats, and covers the region $1.1<|\eta|<1.4$. The scintillator is laid out in 0.5 " thick tiles encased in light-tight Aluminum boxes. Each tile occupies $\Delta \eta \times \Delta \phi \approx 0.3 \times 0.4$. Light from the tiles is fed out of the detector by wavelength shifting (WLS) fibers into photomultiplier tubes (PMT's). 


\subsubsection{Noise in the Calorimeter}

Each cell in the calorimeter is subject to noise both from the electronics, and from signals due to Uranium decay. To account for this noise, the output of each cell is measured in the absence of a beam, this output is referred to as the pedestal of the cell, a typical value would be $\approx 20 \mathrm{GeV}$. The energy of the cell is adjusted up to the mean of the pedestal distribution, this means that a cell can have either positive or negative energy, and the cell is only read out if the energy is above $1.5 \sigma_{\text {ped }}$ where $\sigma_{\text {ped }}$ is the width of the pedestal distribution. Doing this removes most of the normal noise from the calorimeter cells. However, periodically cells will display substantially more noise than expected from their pedestal, with the worst of these registering hundreds of $\mathrm{GeV}$. The T42 algorithm tries to remove these especially noisy cells, while keeping cells with moderate energy close to signal cells [16][17][18]. In the T42 algorithm, cells with an energy greater than $4 \sigma_{\text {ped }}$ are regarded as signal, cells with an energy less than $2.5 \sigma_{\text {ped }}$ are discarded, and cells in-between the two thresholds are kept if they have a neighboring cell with an energy greater than $4 \sigma_{\text {ped }}$

\subsection{Muon System}

The muon system is a large volume tracking detector. In addition to identifying muons exiting the inner part of the detector, the muon system can discriminate cosmic muons using timing information, provide fast information for the level 1 trigger, and provide a momentum measurement which can complement the measurement from the central tracker[19].

The muon system is divided into central and forward parts. The central part forms a rectangular tube around the beam, and provides coverage to $|\eta| \lesssim 1.0$, while the forward part is perpendicular to the beam and extends coverage to $|\eta| \approx 2.0$.

\subsubsection{Toroid Magnet}

There are three toroid magnets that form the center of the muon system, one for the central muon detector, and two for the forward parts. The field inside the toroids is $\approx 1.8 \mathrm{~T}$. 


\subsubsection{Central}

The central muon system is composed of the central toroid magnet, the Proportional Drift Tubes (PDT's), the cosmic cap and bottom scintillation counters, and the $A \phi$ scintillation counters.

The central drift tubes are divided up into three layers, the A layer which sits on the inside of the toroid magnet, and the $\mathrm{B}$ and $\mathrm{C}$ layers which sit on top of one another on the outside of the toroid. Just over half of the central region is covered by all 3 layers, and $90 \%$ is covered by at least 2 layers. The drift tubes are large in size at $2.8 \times 5.6 \mathrm{~m}^{2}$ and made of aluminum. The individual cells are $10.1 \mathrm{~cm}$ across, and a typical chamber is 24 cells wide. Each cell has an anode wire an the center, and vernier cathode pads located above and below the wire to provide information about the location of a hit along the wire.

The Cosmic Cap are scintillators on the top of the muon system. The bottom counters are identical scintillators on the bottom of the detector. They are used primarily to aid in the identification of cosmic muons.

The $A \phi$ scintillator covers the A layer, and provides fast detection which is useful for triggering and rejecting cosmic muons.

\subsubsection{Forward}

The forward muon system is composed of the end toroid magnets, 3 layers of Mini Drift Tubes (MDT's), 3 layers of scintillation counters, and shielding around the beam pipe.

The MDT's have a short drift time compared to the PDT's $\lesssim 132$ ns, good coordinate resolution $\lesssim 1 \mathrm{~mm}$, radiation hardness, high segmentation, and low occupancy.

As in the central muon system, the MDT's are arranged into A,B, and C layers, with the A layer sitting inside the toroid and the B and C layers sitting outside the toroid.

The efficiency is $\approx 100 \%$ in the active area of the MDT's for tracks perpendicular to the plane, and the overall efficiency is $\approx 90 \%$. However, momentum resolution is limited by multiple scattering. For a $40 \mathrm{GeV}$ muon with $\eta=0$ the momentum resolution if found to be $60 \%$ or $\approx 24 \mathrm{GeV}[20]$. 


\subsection{Luminosity Monitor}

The purpose of the luminosity monitor is to determine the instantaneous luminosity of the Tevatron at the $\mathrm{D} \varnothing$ interaction region. It does this by detecting inelastic $p \bar{p}$ collisions in a a dedicated detector. It also measures the beam halo rate, and provides a fast measurement of the $\mathrm{z}$ coordinate of the interaction vertex.

The detector covers the region $2.7<|\eta|<4.4$ and sits at $z= \pm 140 \mathrm{~cm}$, in front of the end calorimeters radial region between the beam pipe and forward preshower detector.

The luminosity is determined by counting the number of inelastic collisions per beam crossing $\bar{N}_{L M}$, and relating that to the luminosity $\mathcal{L}$ through the formula:

$$
\mathcal{L}=\frac{f \bar{N}_{L M}}{\sigma_{L M}}[4]
$$

Where $f$ is the beam crossing frequency and $\sigma_{L M}$ is the effective cross section.

The luminosity monitor requires that both the north and south detectors fire, and the time difference between the two is then used to make a quick determination of the $\mathrm{z}$ position of the primary vertex.

\subsection{Trigger}

The Tevatron delivers beam crossings at a rate of $\approx 2 \mathrm{MHz}$, but it is only possible to store an average of $\approx 50 \mathrm{~Hz}$ of data to tape. To reduce the acceptance rate for events to a suitable level, DØ uses a three level trigger system. The first level (L1) is hardware based, uses only part of the data from the detector, and reduces the rate to $\approx 10 \mathrm{kHz}$. The second level (L2) uses single board computers (SBC's) programmed with firmware, and a slightly expanded set of inputs, to reduce the rate down to $\approx 1 \mathrm{kHz}$. The third and final level (L3), uses a farm of Linux-based computing nodes, and the full readout from the detector, to perform a simplified software reconstruction, to reduce the rate down to $\approx 50 \mathrm{~Hz}$. The data is then be written to tape, and later fully reconstructed offline. A typical event is 250 $\mathrm{kB}$, and over a 12 hour store, a $50 \mathrm{~Hz}$ rate would mean about $1 \mathrm{~TB}$ of data being recorded. 


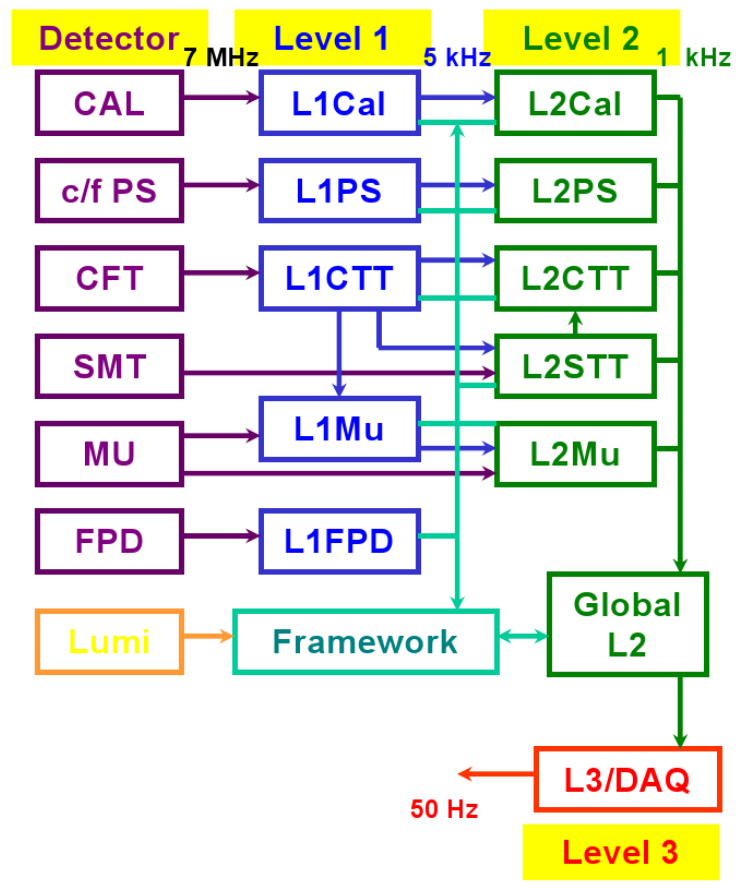

Figure 2.6: Diagram of current DØtrigger system [5].

\section{Trigger Framework}

The trigger framework coordinates the different trigger levels. It accepts digital information from L1 and L2, and decides whether an event should be accepted. The framework is responsible for applying prescales, i.e. accepting only a fixed fraction of events from specific triggers. The framework also coordinates the trigger and readout.

The framework receives up to 256 AND-OR terms, and from these it can form a maximum of 128 triggers. These triggers have both their specific physics requirement and a beam condition, obtained from the luminosity system, in order to fire.

There are 128 geographic sectors, each of which has its own serial command link (SCL), which sends trigger decisions from the trigger framework to the detector components. The individual sectors each receive an L1 accept from the trigger framework through the SCL, followed by an L2 accept or L2 reject from L2 global. Each sector holds data in firstin-first-out (FIFO) buffers, and an event which doesn't receive an accept will simply be 
discarded when it reaches the end of its buffer.

\subsubsection{Level 1}

In Run IIa the L1 trigger system was composed of the L1 Calorimeter trigger (L1CAL), L1 Central Tracking trigger (L1CTT), and L1 Preshower trigger (L1PS). In Run IIb the L1CAL was significantly upgraded, and the L1 Calorimeter Track trigger (L1CalTrk) was added. The L1CalTrk matches L1 tracks to L1 calorimeter clusters, this is useful when triggering on electrons or taus.

\section{L1CAL}

The inputs to the L1 Calorimeter trigger are 1280 Electromagnetic (EM) trigger towers and 1280 Hadronic $(\mathrm{H})$ trigger tower energies. Each tower energy is the sum in depth and transverse coordinates $(\eta \times \phi=0.2 \times 0.2)$ of signals from the Baseline Subtraction (BLS) circuits. The towers are divided into 40 sections in $\eta$, and 32 sections in $\phi$, which make 1280 towers in total. The raw energy read out from the tower is changed to transverse energy, the pedestal energy is subtracted (see Sec. 2.4.2), the energy is adjusted by a constant scale, and finally the transverse energy is fed into the trigger.

In Run2b several significant upgrades were made to the L1CAL. The first of these changes was the addition of a digital filtering algorithm at L1. This filtering takes the output of each calorimeter cell, and tries to remove pileup, i.e. energy from collisions happening before the current beam crossing. The second of these changes was the introduction of sliding window algorithms, which construct clusters from the existing calorimeter trigger towers (see Fig 2.7). The sliding window algorithms allow for the creation of objects in L1CAL which much more closely match physical objects than do the trigger towers. The sliding windows also allow the introduction of shape and isolation cuts at L1.

The L1CAL makes use of predefined thresholds in energy. In Run2a there were four such thresholds in $E_{T}=E \sin \theta$ for each $\mathrm{EM}$ and $\mathrm{EM}+\mathrm{H}$ tower, and 4 thresholds for $\sum E_{T}=\sum E_{T}^{E M}+E_{T}^{H a d}$ and $\not{ }_{T}$. In Run2b the thresholds are based on the transverse 
energies of EM and Jet clusters, rather than the trigger towers.

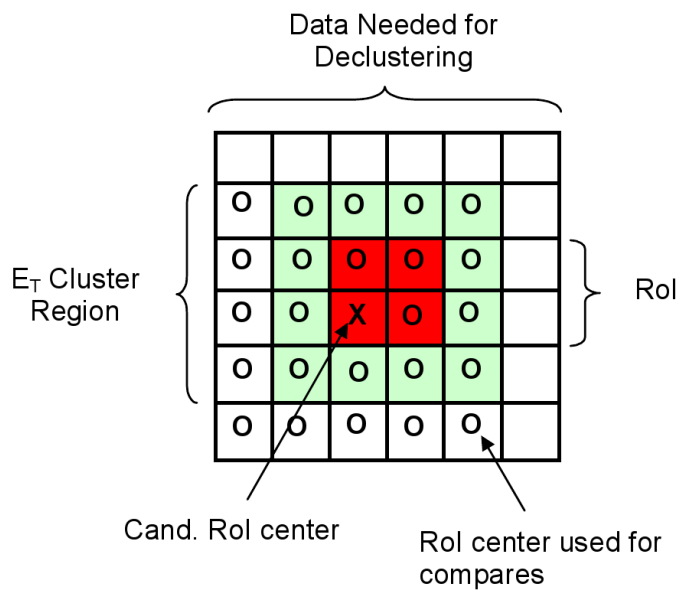

Figure 2.7: Schematic diagram of sliding windows algorithm for jets. Roi stands for region of interest and is the $2 \times 2$ red squares in the center, the sum of the towers in this region is maximized. The $4 \times 4$ region just outside the Roi is used to determine the final $E_{T}$ of the cluster, while the $6 \times 6$ region outside that is used to remove overlapping clusters [5].

\section{L1CTT}

The inputs to the L1CTT are discriminator bits generated by the AFE boards every $132 \mathrm{~ns}$. There are three subsystems, CFT/CPS axial, CPS stereo, and FPS. The CFT/CPS axial and FPS provide L1 trigger terms - all three send track and cluster lists to preprocessors and readout crates.

The CFT/CPS axial subsystem is designed to trigger on charged particles with $p_{T}>$ $1.5 \mathrm{GeV}\left(p_{T}>3 \mathrm{GeV}\right.$ for Run2b). It finds tracks and CPS clusters, matches tracks to clusters, reports the overall occupancy of the CFT axial layers, triggers on isolated tracks, and provides the L1Muon and L2STT systems with lists of seed tracks, and sends track and cluster information to the L2CTT and L2PS systems.

The CFT/CPS axial fibers are grouped by cylinder layer in the AFE's, but the track finder requires that fiber information be arranged in $4.5^{\circ}$ sectors, and each track finder needs information from neighboring sectors. A mixer system takes signals from each sector, and each neighboring sector, and sends that information to the Digital Front End Axial 
boards (DFEA). The DFEA's use track equations grouped into four $p_{T}$ bins, with each $p_{T}$ bin corresponding to a single FPGA. Each track finder FPGA outputs the six highest $p_{T}$ tracks, and these are sent to a fifth FPGA which sorts them, matches tracks to CPS clusters, counts the number of tracks, and calculates the sector occupancy and total $p_{T}$ per sector. Tracks from each sector are sent to the L1Muon system where the tracks are matched to muon hits. The next tier of DFE boards (CTOC) collects and sorts data from within each octant (10 sectors) and checks for isolated tracks. The information for each octant is sent to a single board (CTTT) where trigger terms are generated and sent to the trigger framework within $2.5 \mu$ s of the beam crossings.

On receiving an L1Accept, the AFE boards digitize fiber data from the analog buffers in an analog pipeline. The DFEA's send a sorted list of tracks and CPS clusters to the CTOC, where finer $p_{T}$ information is used to sort the tracks. Then the tracks are sent to L2, and used as seeds for L2STT.

The CPS stereo subsystem provides information on clusters in the two stereo layers of the CPS. It does not provide information to the L1 trigger, but instead stores information for use in L2.

The FPS subsystem is similar in structure to the CFT/CPS axial subsystem.

\section{L1Muon}

The L1Muon system finds patterns consistent with muons from the muon wire chambers, scintillation counters, and L1CTT tracks. FPGA's do the combinatorial logic for the 60,000 muon channels and up to 480 tracks from L1CTT for each bunch crossing.

L1Muon is divided into North, South and Central sections, and further divided into octants. Scintillator trigger cards match L1CTT tracks to muon scintillator hits (MTC06). Wire trigger cards (MTC10) match scintillator confirmed track stubs in wire chambers between 2 or 3 layers of the muons system. Octant decisions of the MTC05/MTC10 pair in a region are summed in muon trigger crate managers (MTCM's) and sent to the muon trigger manager (MTM). The MTM forms 256 global L1Muon triggers, and send up to 32 
of these to the trigger framework. The total latency of L1Muon is $\approx 3.2 \mu s$ is driven by the drift time of the central wire chambers (PDT's), and the time required to form tracks in the L1CTT.

The MTC05 cards match tracks from the L1CTT to hits in the scintillator. Each octant receives L1CTT tracks for ten $4.5^{\circ}$ sectors in that octant plus the adjacent sectors in neighboring octants. Each sector sends the six highest $p_{T}$ tracks to L1Muon. Each track contains CFT fiber position in the outermost layer of the CFT, the $p_{T}$ value of the track and the sign of the track curvature in the central field. The output includes both loose (A-layer only) and tight (A and B layer) tracks.

The MTC10 cards take hits in each layer and form track stubs or centroids. These are then used to confirm scintillator hits in each layer. In the forward region, centroid finding is done by separate cards (MCEN's). The output includes both loose (A-layer) and tight $(\mathrm{A}+\mathrm{B}$ layers $)$ tracks.

\section{L1CALTrk}

The L1CalTrk system was added in Run2b. It uses similar to electronics to L1Muon, and matches clusters from L1CAL to tracks from L1CTT.

\subsubsection{Level 2}

The L2 trigger consists of detector specific preprocessing engines and a global stage (L2Global) to test for correlations in physics signatures across detector subsystems. The system receives inputs from $\mathrm{L} 1$ at $\approx 10 \mathrm{kHz}$, and has a maximum accept rate of $1-2 \mathrm{kHz}$. L2 forms physics objects from front-end data from the L1 trigger system. Events passing L2 are tagged for full readout and passed to L3.

L2CAL uses a clustering based on L1 towers in Run2a, and L1 clusters in Run2b.

L2CTT takes input from L1CTT and L2STT, and creates 2 lists of tracks, one sorted by $p_{T}$ and the other by impact parameter.

The L2 silicon track trigger (STT) adds SMT hit information to L1CTTtracks. This 
allows for the measurement of the impact parameters of tracks with a resolution of $\sim$ $20 \mu \mathrm{m}$, precise enough to tag the decay of long lived particles like B-hadrons. The hits from the CFT are connected to SMT hits within a road and utilizes only the axial strips in the SMT.

L2Muon improves L1Muon using improved calibration and more precise timing information. It outputs $p_{T}, \eta, \phi$, quality and timing information.

L2Global selects events based on 128 L1 terms plus extra conditions.

\subsubsection{Level 3}

The L3 trigger is a high level, fully programmable software trigger. It performs a limited reconstruction of events. The input rate of $1 \mathrm{kHz}$ is reduced to $50 \mathrm{~Hz}$. Decisions are based on complete physics objects, and also on $\delta \eta, \delta \phi$, the invariant mass of two objects. The filter tools unpack raw data, find hits, form clusters, apply calibration, and reconstruct electrons, muons, taus, jets, vertices, and missing transverse momentum.

Events passing L3 are sent to the Datalogger (located at DØ), where they are written to disk for temporary storage. Later the data is transferred to tapes for long term storage at the Feynman Computing Center (FCC). 


\section{Chapter 3}

\section{Object Reconstruction}

The signals left in the detector must be used to reconstruct physical objects: electrons, muons, jets, and taus. It is then essential to calibrate the measured energy or momentum of these objects to the true energy, and understand the resolution of this measured energy or momentum.

\subsection{Track Reconstruction}

Charged particles moving through the detector leave behind signals or hits in the detectors. We must first use these hits to identify the high momentum charged particles in the event. To accurately measure the original momentum of these particles requires a sophisticated understanding of the forces affecting the particles as they pass through the detector, and after doing so there will be an experimental resolution which has to be measured from the data.

\subsubsection{Motion of a Charged Particle in the DØ detector}

The central solenoid magnet of the $\mathrm{D} \varnothing$ detector generates a fairly uniform $2 \mathrm{~T}$ magnetic field. Charged particles traveling through this magnetic field will experience the usual magnetic force: 


$$
\frac{d \vec{p}}{d t}=q \vec{v} \times \vec{B}
$$

If we had no material in our detector, we could determine the momentum of a charged particle by measuring the sagitta of the arc of the track (fig. 3.1):

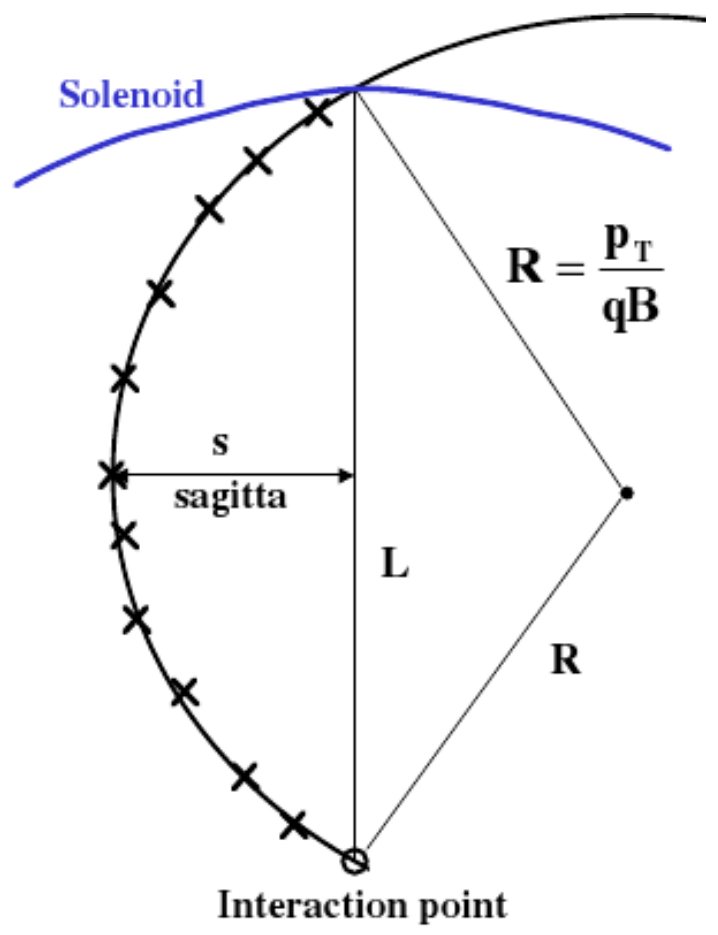

Figure 3.1: Charged particle traveling through solenoid magnetic field.

$$
s=\frac{L^{2}}{8 R}
$$

The radius of the particles trajectory is given by: $R=\frac{p_{T}}{q B}$, and the momentum perpendicular to the magnetic field can be measured as:

$$
p_{T}=\frac{q B L^{2}}{8 s}
$$

From this formula, we see that:

$$
\sigma\left(1 / p_{T}\right)=\frac{8 \sigma(s)}{q B L^{2}}=\frac{\sigma\left(p_{T}\right)}{p_{T}^{2}}
$$


These formulae are useful as a first approximation, and tells us that the momentum resolution of the tracker deteriorates quickly at high $p_{T}$.

In the real detector, there is material in the tracker. Particles interact with the material through the electromagnetic force and as a result they loose energy and get scattered.

Energy loss for charged particles moving through matter is due to the ionization and excitation of electrons in the material. This energy loss can be calculated via the BetheBloch equation [6]:

$$
\frac{d E}{d x}=-4 \pi N_{0} r_{e}^{2} m_{e} c^{2} \rho \frac{Z}{A} z^{2} \frac{1}{\beta}\left[\frac{1}{2}\left(\frac{2 m_{e} c^{2} \beta^{2} \gamma^{2} T_{\max }}{I^{2}}\right)-\beta^{2}-\frac{\delta(\beta \gamma)}{2}\right]
$$

Where: $N_{0}$ is Avogadro's Number, $r_{e}$ is the Classical electron radius, $m_{e}$ is the Electron mass, $\rho$ is the density of the material, $Z$ is the Atomic number of the material, $A$ is the Atomic mass of the material, $\gamma=1 / \sqrt{1-\beta^{2}}, \delta(\beta \gamma)$ is the density effect correction, and $T_{\max }$ is the maximum kinetic energy which can be imparted to a free electron in a single collision given by:

$$
T_{\text {max }}=\frac{2 m_{e} c^{2} \beta^{2} \gamma^{2}}{1+\frac{2 \gamma m_{e}}{M}+\left(\frac{m_{e}}{M}\right)^{2}} .
$$

Which in the limit of $\gamma<<\frac{M}{m_{e}}$ becomes: $T_{\max } \approx 2 m_{e} c^{2} \beta^{2} \gamma^{2}$.

From Eqn. 3.5 we see that the energy loss experienced by a particle is dependent on its velocity $\beta$. From Fig. 3.2 we see that there is broad region near the point of minimum ionization where the energy loss remains relatively low. At DØ most of the charged particles produced have energies within the minimum-ionizing region, and the energy loss is approximated as a linear function of the energy of the particle [21]:

$$
\Delta E=(-1.66-0.0138 E) \rho x .
$$

Where $\Delta E$ is measured in $\mathrm{MeV}$, the material density $\rho$ is measured in $\mathrm{gcm}^{-3}$, and the distance traveled through the material $x$ is measured in $\mathrm{cm}$. The constant $1.66 \mathrm{MeVcm}^{2} \mathrm{~g}^{-1}$ is an average value of the minimum rate of energy loss of particles in different materials. The energy dependent factor is tuned using the GEANT [22] simulation of the DØ detector. 
Multiple scattering is due principally to coulomb interactions with the nuclei of the material. The gaussian width of the scattering angle is given by [6]:

$$
\delta \theta \approx \theta_{0}=\frac{13.6 \mathrm{MeV}}{\beta c p} z \sqrt{\frac{x}{X_{0}}}\left[1+0.038 \ln \frac{x}{X_{0}}\right]
$$

Where $p$ is the particle momentum, $\beta c$ is the velocity, $z$ is the charge number of the incident particle, $\frac{x}{X_{0}}$ is the thickness of the scattering material in radiation lengths. This width can be used either randomly alter the trajectory of a simulated charged particle, or can be used to increase the parameter errors when propagating a reconstructed track through the detector.

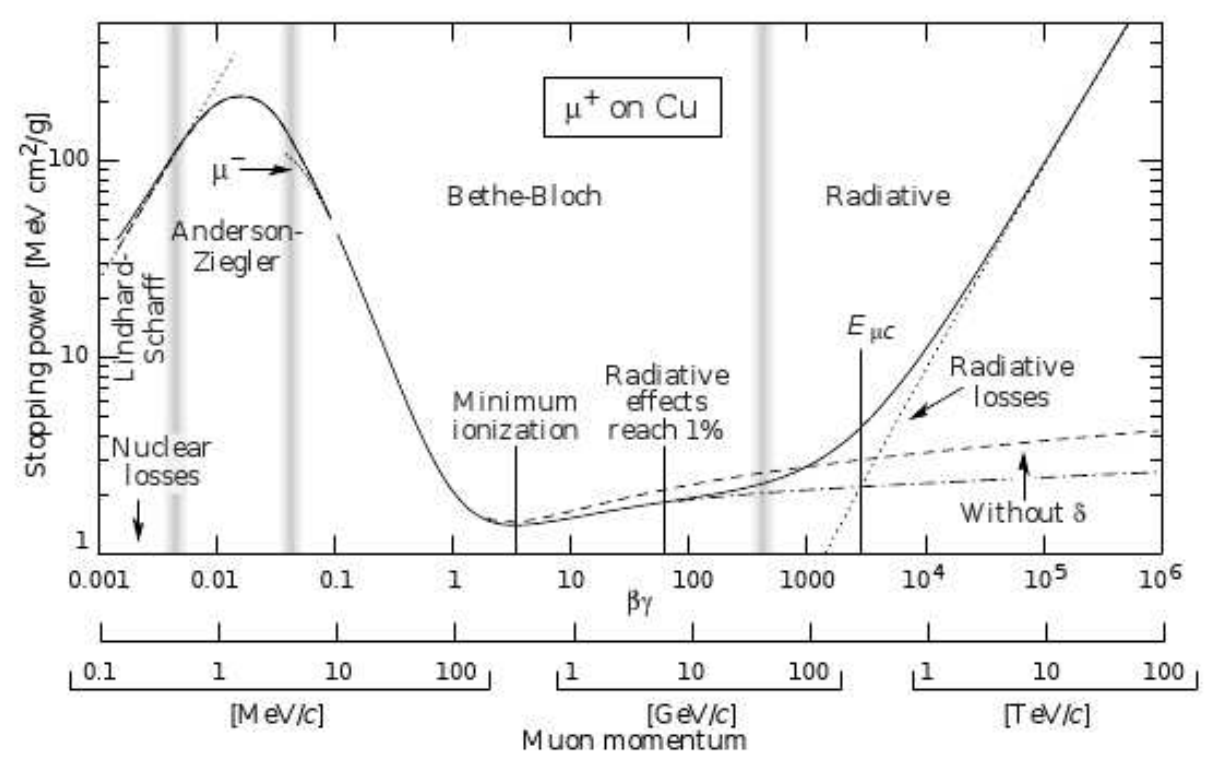

Figure 3.2: Energy loss for muons traveling through copper [6].

\subsubsection{Track Finding Algorithms}

There are two main tracking algorithms, AA [7], and HTF [8] (Histogramming Track Finder).

AA uses a road finding method. A set of three 2-D hits $(r, \phi)$ in the SMT is used to form a track candidate. These candidates are then propagated out through the remaining 
layers of the SMT and into the CFT, in each layer the hits within a test region are tested on the candidate track and if the resulting $\chi^{2}$ of the modified candidate is below a certain value the candidate is modified by adding the new hits (fig. 3.3).

HTF starts with pairs of hits in either the CFT or SMT. Assuming that the track originated at the beam spot, the pair of hits can be transformed into a line in curvature and $\phi\left(q B / p_{T}, \phi\right)$ space, this process is known as a Hough Transform. The curvature-phi space is divided up into a 2-dimensional histogram. Each bin of the histogram that a line passes through is incremented by one, and this is repeated for the different pairs of hits. Bins are removed from consideration if they don't have a minimum number of hits. The cells that remain are used as templates, that is they are used to define a set of hits to look at. This procedure is done starting in the SMT and projecting to the CFT, as well as the other way around (fig. 3.4).

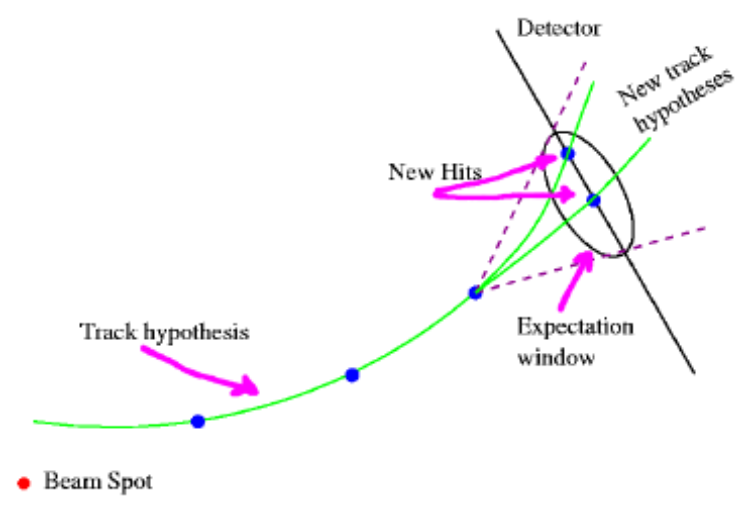

Figure 3.3: Extension of track hypothesis to new layer of detector [7].

\subsubsection{Track Momentum Resolution}

The track resolution is expected to take the form:

$$
\frac{\sigma\left(p_{T}^{-1}\right)}{p_{T}^{-1}}=\sqrt{A^{2} p_{T}^{2}+B^{2} \cosh \eta} .
$$

Where $A$ derives from the position resolution of the detector, and $B$ reflects the impact of multiple scattering due to interaction with the detector. 

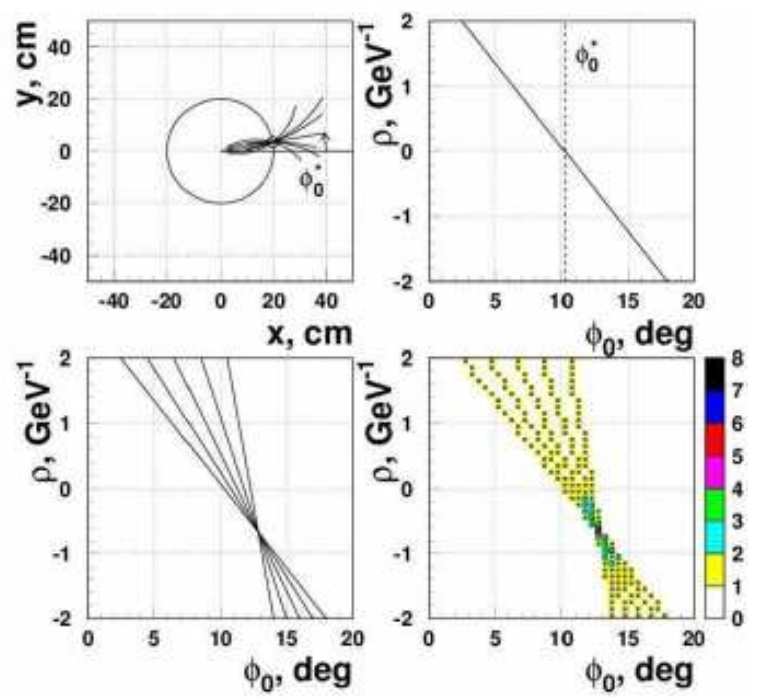

Figure 3.4: Upper left: Track hypotheses. Upper right: Line in $\rho, \phi$ for single hypothesis. Lower left: Several hypotheses. Lower right: Histogramming of hypotheses [8].

For tracks without any SMT hits, the $p_{T}$ is corrected using the primary vertex as a constraint. The correction takes the form:

$$
\frac{q}{p_{T}^{\prime}}=\frac{q}{p_{T}}-d c a \times \frac{\sigma_{r, q / p_{T}}}{\sigma_{r, r}}
$$

Where $q$ is the charge of the track, $d c a$ is the distance of closest approach between the track and the primary vertex, and $\sigma$ are elements of the track error matrix.

The resolution parameters have been measured using two processes: $J / \psi \rightarrow \mu^{+} \mu^{-}$and $Z \rightarrow \mu^{+} \mu^{-}$. The masses of these two resonances are quite different $\left(m_{J / \psi}=3.096 \mathrm{GeV}\right.$, $m_{Z}=91.1876 \mathrm{GeV}$ ), and they involve muons with substantially different momenta, which allows for a much better determination of the momentum dependence of the resolution parameters [23][24]. 
Table 3.1: Parameters for the Muon Resolution.

\begin{tabular}{lccc}
\hline muon type & run range & $A$ & $B$ \\
\hline$N_{S M T}>0$ and $\left|\eta_{C F T}\right|<1.6$ & run $<200000$ & $2.3 \times 10^{-3}\left[\mathrm{GeV}^{-1}\right]$ & $2.3 \times 10^{-2}$ \\
& $200000<$ run $<220000$ & $2.6 \times 10^{-3}\left[\mathrm{GeV}^{-1}\right]$ & $2.4 \times 10^{-2}$ \\
& run $>220000$ & $2.3 \times 10^{-3}\left[\mathrm{GeV}^{-1}\right]$ & $2.5 \times 10^{-2}$ \\
\hline$N_{S M T}>0$ and $\left|\eta_{C F T}\right|>1.6$ & run $<200000$ & $4.3 \times 10^{-3}\left[\mathrm{GeV}^{-1}\right]$ & $3.4 \times 10^{-2}$ \\
& $200000<$ run $<220000$ & $4.8 \times 10^{-3}\left[\mathrm{GeV}^{-1}\right]$ & $3.5 \times 10^{-2}$ \\
& run $>220000$ & $3.9 \times 10^{-3}\left[\mathrm{GeV}^{-1}\right]$ & $3.7 \times 10^{-2}$ \\
\hline$N_{S M T}=0$ & run $<200000$ & $3.7 \times 10^{-3}\left[\mathrm{GeV}^{-1}\right]$ & $3.5 \times 10^{-2}$ \\
& $200000<$ run $<220000$ & $4.1 \times 10^{-3}\left[\mathrm{GeV}^{-1}\right]$ & $3.5 \times 10^{-2}$ \\
& run $>220000$ & $3.7 \times 10^{-3}\left[\mathrm{GeV}^{-1}\right]$ & $3.3 \times 10^{-2}$ \\
\hline
\end{tabular}

\subsection{EM Cluster Reconstruction}

\subsubsection{Electromagnetic Showers}

High momentum electrons traveling through material will interact with the electrons and nuclei of the material. When the momentum transfer of this interaction is small, the result will be a steady loss of energy or scattering through a small angle (see section 3.1.1). Larger momentum transfers lead to the emission of high momentum photons. The distance after which an electron has $1 / e$ of its original energy left is given by the radiation length $X_{0}$. High momentum photons traveling through material can produce electron positron pairs by exchanging energy and momentum with atoms of the material. The mean free path for $e^{+} e^{-}$pair production is $7 / 9$ of the radiation length $X_{0}$.

Electromagnetic showers are produced when a high momentum electron or photon enters the material of the detector, and initiates the emmission of a photon or the production of an electron positron pair. The resulting particles then repeat the same process, and the whole thing continues until the final electrons and photons no longer have sufficient energy to initiate new interactions 


\subsubsection{Electromagnetic Cluster Reconstruction}

To reconstruct electrons, we first find electromagnetic clusters in the calorimeter. Clusters are formed from seed towers with $p_{T}>500 \mathrm{MeV}$. Neighboring cells are added if they have $p_{T}>50 \mathrm{MeV}$ and are within $\Delta R=\sqrt{(\Delta \eta)^{2}+(\Delta \phi)^{2}}<0.3$ in the $\mathrm{CC}$, or within a cone of $10 \mathrm{~cm}$ in the third EM layer in the EC, and the result is referred to as a precluster. Preclusters with $p_{T}>1 \mathrm{GeV}$ are used as the starting point for the final clusters. Any EM tower within $\Delta R<0.4$ is added to the cluster.

These EM clusters result from EM showers, and those showers can be initiated by either electrons or photons. We are only interested in those showers initiated by high $p_{T}$ electrons, and we want to eliminate showers coming from[25][26]:

- $\pi^{0} \rightarrow \gamma \gamma$ incorrectly matched to a track.

- Photons which convert to $e^{+} e^{-}$.

- Charged pions that undergo charge exchange in the detector.

- Fluctuations of QCD shower shapes.

While there are many different variables that can be used to discriminate electrons from these backgrounds, to maximize the discrimination power, we use a likelihood of the form $[25][26][27]$ :

$$
\mathcal{L}=\frac{\prod_{i=1}^{n} P D F_{e}(i)}{\prod_{i=1}^{n} P D F_{e}(i)+\prod_{i=1}^{n} P D F_{q c d}(i)} .
$$

Eight different variables are used as inputs to this likelihood:

- Number of tracks within a cone of $\Delta R<0.4\left(N_{t r k}\right)$

- Scalar sum of track $p_{T}$ 's in a hollow cone of $0.05<\Delta R<0.4\left(\sum p_{T}^{\operatorname{trk}}\right)$

- Track spatial matching probability, probability calculated from spatial $\chi_{\text {spatial }}^{2}=$ $\left(\frac{\Delta \eta}{\sigma_{\eta}}\right)^{2}+\left(\frac{\Delta \phi}{\sigma_{\phi}}\right)^{2}$ 
- E over p : $\frac{E_{T}^{E M}}{p_{T}^{\text {track }}}$, where $p_{T}^{\text {track }}$ is corrected if $N_{S M T}=0$

- Distance of closest approach between charged track and Primary Vertex (dca)

- HMatrix-7 (HMx7), and HMatrix-8 (HMx8) : The two variables are $\chi^{2}$ values comparing the observed shower shape, to that expected from the Monte Carlo. The HMx7 uses seven variables: the fraction of energy in each of the four layers of the EM calorimeter, the width of the shower in $\Delta R$, the log of the total shower energy, and the longitudinal position of the events primary vertex. The HMx8 adds to this either the either the size of the cluster in $\mathrm{z}$ for the central region, or the size of the cluster in $\mathrm{r}$ for the forward region.

In our analysis we use two electron definitions, a loose definition which removes some of the fake-electron events, and a tight definition which removes most of the fake-electron events.

The loose definition is called Top_loose, and requires:

- $f_{\text {iso }}=\frac{E_{t o t}(\Delta R<0.4)-E_{E M}(\Delta R<0.2)}{E_{E M}(\Delta R<0.2)}<0.15$

- $E_{t o t}(\Delta R<0.4)$ is the total energy the calorimeter in a cone of $\Delta R<0.4$.

- $E_{E M}(\Delta R<0.2)$ is the energy in the EM calorimeter in a cone of $\Delta R<0.2$.

- $f_{E M}=\frac{E_{E M}}{E_{T o t}}>0.9$

- $\operatorname{HMx} 7<50$

- Track spatial matching probability with EOP (Energy over Momentum), $\chi_{E O P}^{2}=$ $\left(\frac{\Delta \eta}{\sigma_{\eta}}\right)^{2}+\left(\frac{\Delta \phi}{\sigma_{\phi}}\right)^{2}+\left(\frac{E_{T} / p_{T}-1}{\sigma_{E_{T} / p_{T}}}\right)^{2} \geq 0.0$ (a negative $\chi^{2}$ means a non-converging fit), in Run2b this was changed to $\chi_{\text {spatial }}^{2}=\left(\frac{\Delta \eta}{\sigma_{\eta}}\right)^{2}+\left(\frac{\Delta \phi}{\sigma_{\phi}}\right)^{2} \geq 0$ and $E_{T} / p_{T}<2.5$ for electrons with $|\eta|<1.1$

- $p_{T}^{\text {track }}>5 \mathrm{GeV}$

The tight definition is called Top_tight. It has the same requirements as Top_loose and also requires that the 8-parameter likelihood defined above have $\mathcal{L}>0.85$ 


\subsubsection{Electron Energy Resolution}

The energy resolutions of electrons has been found to take the form [1]:

$$
\frac{\sigma_{E}}{E}=\sqrt{C^{2}+\frac{S^{2}(E, \theta)}{E}+\frac{N^{2}}{E^{2}}}
$$

Where $C$ is a constant term, i.e. it gives a constant resolution as a fraction of the total energy, $S(E, \theta)$ is the sampling term, coming from the fact that we sample only a portion of the energy in the calorimeter, and $N$ is the noise term. The values for $C$ and $N$ are given in Table 3.2 .

Table 3.2: Parameters for the Electron Resolution [1].

\begin{tabular}{cccc}
\hline & CC & ECN & ECS \\
\hline$C$ & 0.028 & 0.0325 & 0.0278 \\
$N$ & 0.4 & 0.125 & 0.125 \\
\hline
\end{tabular}

The sampling term is found to have a different dependence on $E$ and $\theta$ in the CC and EC:

$$
\begin{aligned}
& S_{C C}(E, \theta)=E \times p_{0}(E) \times \exp \frac{p_{1}(E)}{\sin \theta}-p_{1}(E) \\
& S_{E C}(E, \theta)=E \times p(\eta) \times \frac{s_{0}(\eta) / \sqrt{E}+s_{1}(\eta) / E}{s_{0}(\eta) / \sqrt{45}+s_{1}(\eta) / 45} .
\end{aligned}
$$

Where:

$$
\begin{aligned}
& p_{0}(E)=\frac{0.164}{\sqrt{E}}+\frac{0.122}{E}, \\
& p_{1}(E)=1.35193-\frac{2.09564}{E}-\frac{6.98578}{E^{2}} .
\end{aligned}
$$

For the ECS region:

$$
\begin{aligned}
p(\eta)= & 14.808-53.358 \eta+80.874 \eta^{2}-66.687 \eta^{3}+32.331 \eta^{4} \\
& -9.222 \eta^{5}+1.434 \eta^{6}-0.094 \eta^{7}, \\
s_{0}(\eta)= & 0.217+0.003 \eta-0.007 \eta^{2}, \\
s_{1}(\eta)= & 57.247-104.577 \eta+71.148 \eta^{2}-21.127 \eta^{3}+2.306 \eta^{4} .
\end{aligned}
$$


While for the ECN region:

$$
\begin{aligned}
p= & -9.507+27.044(-\eta)-31.337 \eta^{2}+19.008\left(-\eta^{3}\right)-6.364 \eta^{4} \\
& +1.115\left(-\eta^{5}\right)-0.080 \eta^{6}, \\
s_{0}= & 0.221-0.025(-\eta)+0.002 \eta^{2} . \\
s_{1}= & 9.479-21.201(-\eta)+17.503 \eta^{2}-6.027\left(-\eta^{3}\right)+0.734 \eta^{4}[1] .
\end{aligned}
$$

\subsection{Jet Reconstruction}

\subsubsection{Hadronic Showers}

Quarks and gluons produced with high momentum will undergo a process of gluon emission and quark pair production similar to the process resulting in electromagnetic showers. At first, this process involves a relatively large momentum transfer, and can be described perturbatively. But, as the showering progresses, the coupling grows and the process becomes non-perturbative. At this point the color-charged quarks group themselves into color-neutral hadrons, a process referred to as hadronization. This shower of hadrons is referred to as a jet.

This collection of hadrons tends to include a large fraction $(1 / 3)$ or $\pi^{0}$ 's. These quickly decay to photons, leading to electromagnetic showers. A small fraction of the charged hadrons will undergo decay into $e \nu_{e}$ and $\mu \nu_{\mu}$ pairs, before reaching the detector. Some of the hadrons will undergo decay into $e \nu_{e}$ and $\mu \nu_{\mu}$ pairs, before reaching the detector. The remaining hadrons, mostly $\pi^{+}$'s, and kaons, will interact with nuclei in the detector, leading to more hadrons, and to large energy depositions.

The hadronic showers produced by jets are longer, wider, and have greater fluctuations in shape and composition than do electromagnetic showers. A sizable portion of the energy in the original jet is consumed by delayed emission of photons in nuclear reactions, soft neutrons, and nuclear binding energies.

Special algorithms are needed to reconstruct jets from the signals left in the detector, 
and to correct the energy measured in the detector for various known losses.

\subsubsection{Jet Reconstruction Algorithm}

As described in [28], an ideal jet algorithm must have:

- Infrared and Collinear safety: The algorithm should not create infrared or collinear singularities in perturbative calculations, these arise if the algorithm is sensitive to the soft or collinear radiation of gluons.

- Boundary stability: The kinematic variables that describe the jet should have kinematic boundaries that are insensitive to the details of the final state.

- Order independence: The algorithm should find the same jets at parton, particle, and detector level.

- Detector independence: The algorithm should be reasonably independent of the detector that provides the data.

- Minimization of resolution smearing and bias.

- Stability with growing luminosity.

- Maximal reconstruction efficiency.

- Ease of calibration.

We use the Improved Legacy Cone Algorithm[29]. The algorithm starts with cells in the calorimeter. Noisy cells are removed as in section 2.4.2. The remaining cells are grouped into pseudo-projective towers (shaded black and white in fig. 2.5). Each cell is assumed to have mass zero, and has the 4-momenta: $p_{\text {cell }}^{\mu}=\left(E_{\text {cell }}, E_{\text {cell }} \hat{\mathbf{n}}\right)$ (where $\hat{\mathbf{n}}$ is a unit vector pointing from the interaction vertex, as reconstructed by the tracking system, to the center of the cell), and each tower is given a 4-momentum: $p_{\text {tower }}^{\mu}=\sum p_{\text {cell }}^{\mu}$.

The towers with $p_{T}>1 \mathrm{GeV}$ are used as seeds to find preclusters, which are formed by adding neighboring towers within $\Delta R<0.3$ of the seed towers. The preclusters are then 
used as seeds in the construction of protojets. A cone of $\Delta R<0.5$ is formed around the protojet, all towers within the cone are added together to form a new proto-jet candidate until the protojet is stable, that is until the candidate and the original are within $\Delta R<$ 0.001, then the protojet is added to the list of protojets, and the process iterates until there are no more seeds to consider. Finally, the list of midpoints between preclusters within $0.5<\Delta R<1$, are used as seeds for protojets.

Next, we need to ensure that a tower is not allowed to belong to more than one jet. We compare two jets that overlap, if more than half of the energy of the lower $p_{T}$ jet is shared by the higher $p_{T}$ jet, then the two jets are merged together, otherwise, the towers that are shared between the two jets are assigned to whichever jet they are closer to. After splitting and merging jets, those jets with $p_{T}<6 \mathrm{GeV}$ are discarded.

\subsubsection{Jet Energy Scale}

We need to determine the relationship between the energy of a jet measured in the calorimeter, and the true energy of the jet. We determine this scale using events with a photon and a jet $(\gamma+j e t)$ produced back to back in $\phi$. This allows us to calibrate jets in our data and our simulation using the same procedure [9][30].

The jet energy scale (JES) is parametrized in the following way:

$$
E_{\text {jet }}^{\text {corrected }}=\frac{E_{\text {jet }}^{\text {measured }}-E_{O}}{R_{\text {jet }} S_{\text {jet }}}[9][30] .
$$

- $E_{\text {jet }}^{\text {corrected }}$ is the corrected energy of the jet

- $E_{j e t}^{\text {measured }}$ is the energy of the jet measured in the calorimeter

- $E_{O}$ is the offset correction and corrects for energy not related to the jet

- $R_{j e t}$ is the response correction and accounts for the energy response of the calorimeter to the jet

- $S_{j e t}$ is the showering correction and corrects for energy loss due to out of cone showering 


\subsubsection{Single Pion Response}

The JES described above is derived separately in data and Monte Carlo. The need for a separate derivation is understood to arise from a difference in the response of the calorimeter in data and Monte Carlo. The response in Monte Carlo can be directly measured, and an attempt was made in [9] to derive an in-situ single-pion response in data. This was done by creating a dedicated Monte Carlo where the cell-level energy deposited by hadrons was scaled down to reproduce the jet response in data. The scaling factor depends on the true hadron energy $E_{h}$ and is defined as:

$$
k\left(E_{h} ; A, B, C\right)=R_{\pi}^{D a t a}\left(E_{h} ; A, B, C\right) / R_{\pi}^{M C}\left(E_{h}\right) .
$$

Where $R_{\pi}^{M C}$ is the response measured in Monte Carlo, and has the form suggested in [31]:

$$
R_{\pi}^{M C}\left(E_{h}\right)=c_{2}\left[1-a_{2}\left(\frac{E_{h}}{E_{0}}\right)^{m_{2}-1}\right] .
$$

Where $E_{0}=0.75 \mathrm{GeV}, a_{2}=0.588, m_{2}=0.456$, and $c_{2}=0.870$.

The Data response is assumed to have the same functional form as the Monte Carlo response:

$$
R_{\pi}^{\text {Data }}\left(E_{h}\right)=c_{1}\left[1-a_{1}\left(\frac{E_{h}}{E_{0}}\right)^{m_{1}-1}\right] .
$$

Where $a_{1}=A \cdot a_{2}, m_{1}=m_{2}+B$, and $c_{1}=C \cdot c_{2}$.

The parameters A,B,C are measured to be: $A=1.8086, B=-0.1748$, and $C=$ $0.999[9]$.

In figure 3.5 this parametrized response is compared to a direct measurement of the isolated single pion response directly in data, the two agree quite well for $p_{T}^{\pi}>3 \mathrm{GeV}$. The discrepancy below $3 \mathrm{GeV}$ is thought to arise from zero suppression effects not contributing to the data measurement.

\subsubsection{Sample Dependence Correction}

As we saw in the previous section, the single pion response in data and $\mathrm{MC}$ are significantly different. The JES defined in sec. 3.3.3 explicitly corrects for this difference, but the 

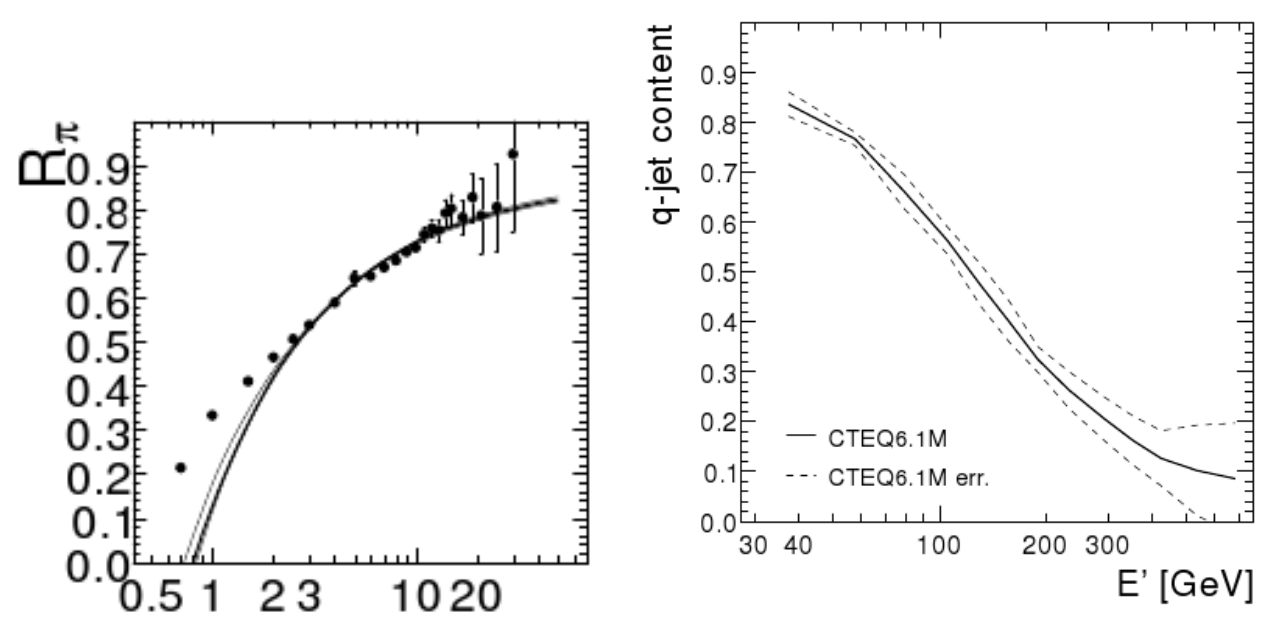

Figure 3.5: Comparison of single pion response derived from data (full circles), and from the fit to the jet response (thick solid line). Quark jet fraction in $\gamma+$ jet events as a function of photon energy [9].

correction is derived on $\gamma+1$ jet events. The single pion response is dependent on the energy spectra of the hadrons in the jet. If the spectra of those hadrons, for a given jet energy, is harder or softer than the average spectra in a $\gamma+j e t$ sample, then applying the standard JES could introduce a bias between data and monte carlo jet energies.

To estimate the size of this bias we use a simplified model of the jet energy scale correction. First we form jets from the particles in the Monte Carlo simulation. The energy of each jet is just the sum of the energies of the particles within it [10]:

$$
E_{\text {partjet }}=\sum_{\text {particle }} E_{\text {particle }}
$$

Now we can model the energy the jet would have in data by multiplying the energy of each particle by the single particle response in data:

$$
E_{0}^{\text {Data }}=\sum_{\text {particle }} R_{\pi}^{\text {Data }}\left(E_{\text {particle }}\right) E_{\text {particle }}
$$

We can then model the energy the jet would have in monte carlo by multiplying the energy of each particle by the single particle response in Monte Carlo:

$$
E_{0}^{M C}=\sum_{\text {particle }} R_{\pi}^{M C}\left(E_{\text {particle }}\right) E_{\text {particle }}
$$


Next we need a simplified model of the $\gamma+$ jet jet energy scale. The correction factors have the form:

$$
\begin{aligned}
& E_{\text {corr }}^{\text {Data }}=C^{\text {Data }} E_{0}^{\text {Data }}, \\
& E_{\text {corr }}^{M C}=C^{M C} E_{0}^{M C}
\end{aligned}
$$

The correction factors are derived such that $E_{\text {corr }}^{\text {Data }}=E_{\text {corr }}^{M C}=E_{\gamma}$, which leads to the following:

$$
\frac{C^{D a t a}}{C^{M C}}=\frac{E_{0}^{M C}}{E_{0}^{D a t a}}
$$

If we apply these correction factors to an arbitrary sample, we expect that, for a given particle jet energy, there will be the following relationship:

$$
E_{\text {corr }, S}^{\text {Data }}=k_{S} E_{\text {corr }, S}^{M C}
$$

We can then write an expression for $k_{S}$, as a double ratio:

$$
k_{S}=\frac{E_{S}^{D a t a}}{E_{S}^{M C}}=\frac{C^{\text {Data }} E_{0, S}^{D a t a}}{C^{M C} E_{0, S}^{M C}}=\frac{E_{0, S}^{\text {Data }} / E_{0, S}^{M C}}{C^{M C} / C^{D a t a}}=\frac{E_{0, S}^{\text {Data }} / E_{0, S}^{M C}}{E_{0, \gamma j}^{D a t a} / E_{0, \gamma j}^{M C}} .
$$

To derive $k_{S}$ we start by forming particle jets using a cone algorithm, the energy of these jets is taken from the sum of the energies of the constituent particles:

$$
E_{\text {part }}=\sum_{i} E_{i}^{\text {particle }}
$$

To obtain $E_{0}^{D a t a}$ and $E_{0}^{M C}$ we apply either the data or monte carlo single pion response derived in [9][30], so that the energies are just:

$$
\begin{aligned}
E_{0}^{\text {Data }} & =\sum_{i} R_{i}^{\pi, \text { Data }} E_{i}^{\text {particle }} \\
E_{0}^{M C} & =\sum_{i} R_{i}^{\pi, M C} E_{i}^{\text {particle }}
\end{aligned}
$$

We can then express $k_{S}$ as a function of $E_{\text {part }}$ and $\eta$ :

$$
k_{S}\left(E_{\text {part }}, \eta\right)=\frac{R_{\text {Sample }}}{R_{\gamma j}}=\frac{E_{\text {part }, S}^{\text {Data }} / E_{\text {part }, S}^{M C}}{E_{\text {part }, \gamma j}^{\text {Data }} / E_{\text {part }, \gamma j}^{M C}}
$$


Figure 3.6 shows $k_{S}$ for light jets in a $t \bar{t}$ sample, while Fig. 3.7 shows $k_{S}$ for b-quark jets in a $t \bar{t}$ sample. From these plots we see a bias of less than $1 \%$ for light jets, and a bias on the order of $2 \%$ for b-quark jets.
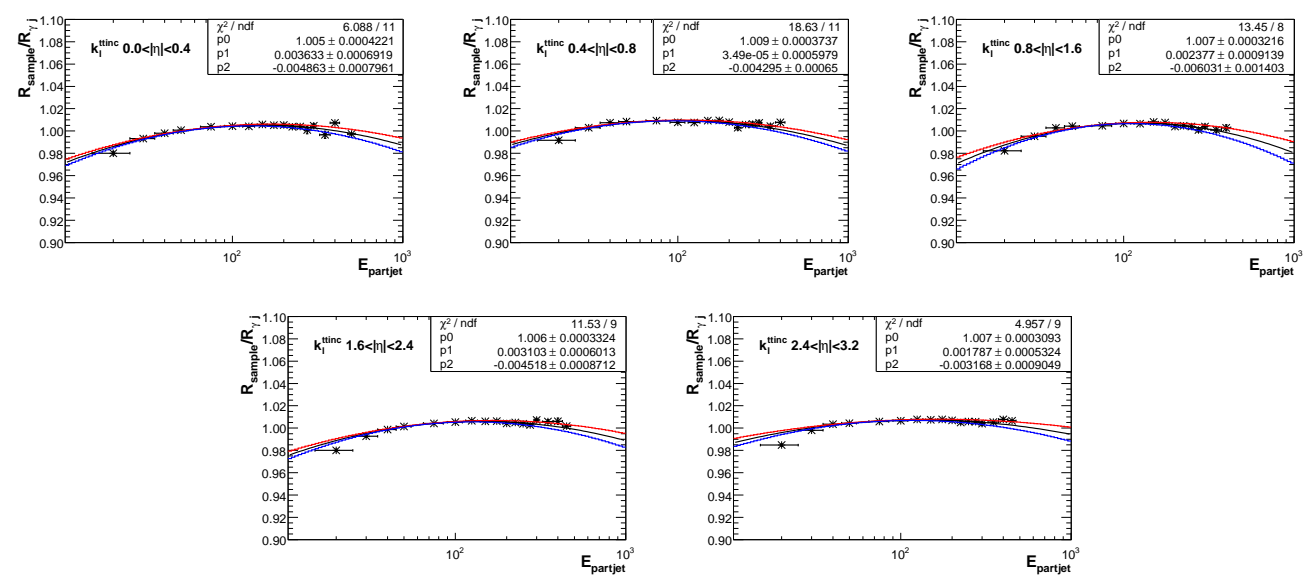

Figure 3.6: $k_{t \bar{t}}^{l}$ for light jets in various $|\eta|$ regions [10].
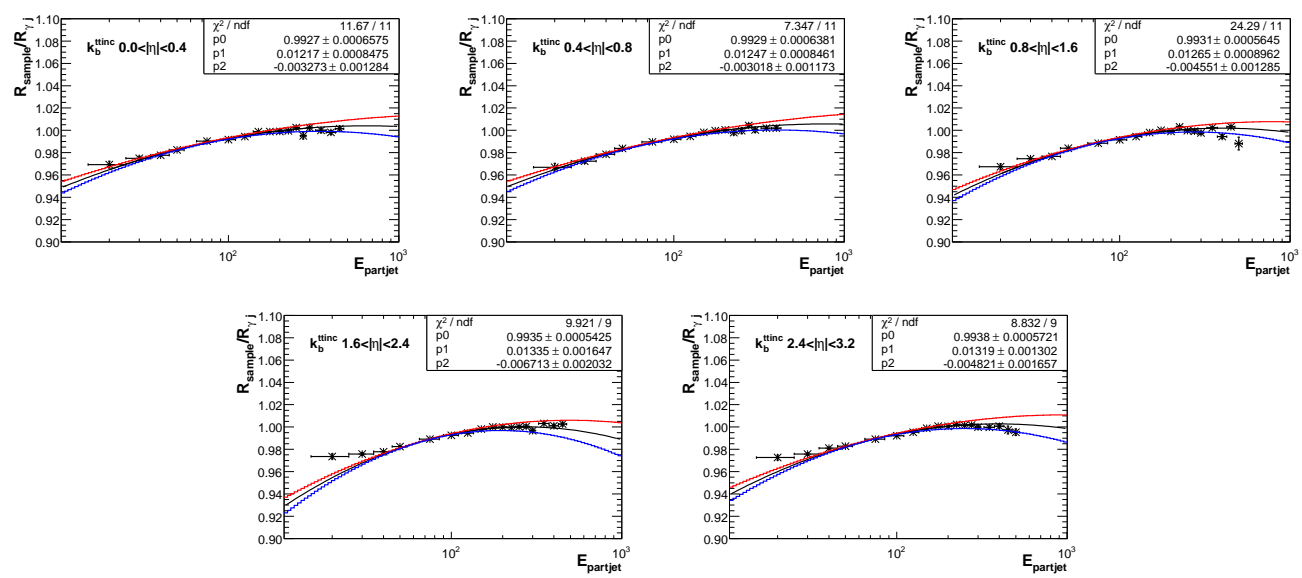

Figure 3.7: $k_{t \bar{t}}^{b}$ for b jets in various $|\eta|$ regions [10].

\subsubsection{Jet Energy Resolution}

The $p_{T}$ resolution for jets has the form:

$$
\frac{\sigma\left(p_{T}\right)}{p_{T}}=\sqrt{\frac{N^{2}}{p_{T}^{2}}+\frac{S^{2}}{p_{T}}+C^{2}} .
$$


The resolution has been measured using the same $\gamma+$ jet sample used to measure the Jet Energy Scale [32][11], see Fig. 3.3. This study used the momentum balance:

$$
\Delta S=\frac{p_{T}^{j e t}-p_{T}^{\gamma}}{p_{T}^{\gamma}} .
$$

Where $p_{T}^{\text {jet }}$ has been corrected with the standard JES. As can be seen in Fig. 3.8, the resolution is greatest at low $p_{T}$, where calorimeter noise dominates, and tends towards a constant at high energies.
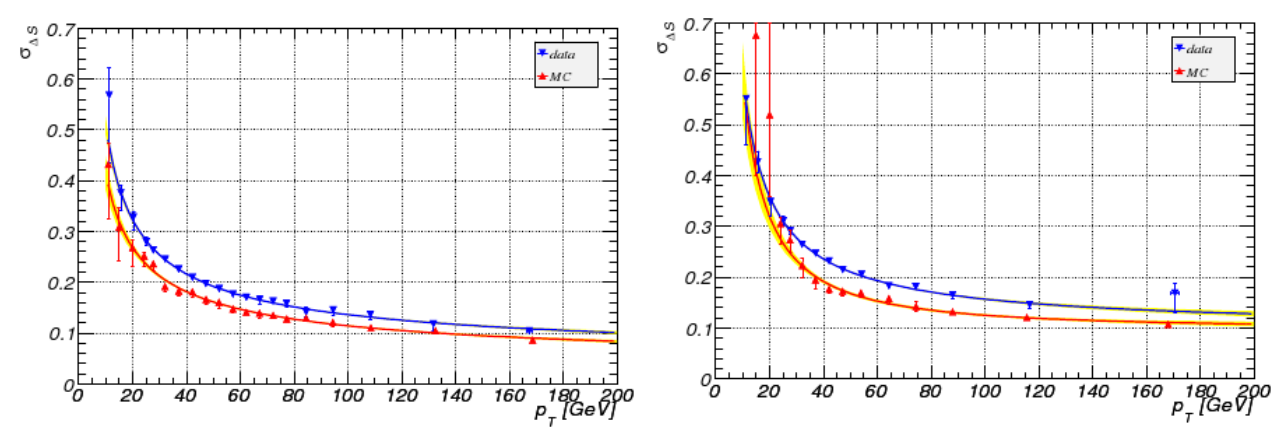

Figure 3.8: Jet Energy Resolution for Data and Monte Carlo in the CC(left) and not CC(right) [11].

Table 3.3: Parameters for the Jet Energy Resolution.

\begin{tabular}{lccc}
\hline & $\mathrm{C}$ & $\mathrm{S}$ & $\mathrm{N}$ \\
\hline$|\eta| \leq 0.8$ & 0.062 & 1.144 & 3.550 \\
$0.8<|\eta| \leq 1.6$ & 0.110 & 0.814 & 6.322 \\
$1.6<|\eta| \leq 2.4$ & 0.0 & 1.302 & 2.920 \\
$2.4<|\eta| \leq 3.6$ & 0.063 & 1.108 & 6.075 \\
\hline \hline
\end{tabular}

\subsection{Muon Reconstruction}

\subsubsection{Muon Reconstruction Algorithm}

The muon system has three layers of detectors (A,B,C, see Section 2.5). Muons are reconstructed by forming segments within each layer, and then matching those segments to segments in the other layers. Muons reconstructed in this way are referred to as local 
muons. These local muons are then matched to tracks reconstructed in the central tracking detectors, if the match is successful the muon is called a central track matched muon.

It is also possible to identify muons by looking for a MIP (minimum ionizing particle) signature in the calorimeter, this algorithm is called "Muon Tracking in the Calorimeter" (MTC). These muons in the calorimeter can be used in the final selection, but only muons that interact with the muon system are used for triggering events.

\subsubsection{Muon Identification}

\section{Muon Quality from Muon System}

Muon quality is specified by two quantities, tightness and the number of segments in the muon system (nseg).

There are two nseg definitions used in this analysis:

- $n s e g=3$ : A local muon with a track running through the A, B, and C layers of the muon system, matched to a central track.

- $n s e g \geq 0$ : At least one hit in the muon system or MTC matched to a central track.

There are two muon quality definitions used in this analysis:

- Medium $\mid n$ seg $\mid=3$ muon: Requires 2 wire and 1 scintillator hit in both A and BC layers matched to a central track.

- Loose muon: Requires 2 wire and 1 scintillator hit in the A layer matched to a central track.

There are two types of muon definitions used: the first is relatively tight and used for muons from semileptonic decays of hadrons, Medium $n s e g=3$, the second definition is looser and is for muons originating from the leptonic decay of $W$ bosons, or the leptonic decay of $t$ leptons, Loose $n s e g>0$. In Run IIa the Medium $n s e g=3$ quality was used for both types of muons, in Run IIb the loose quality was introduced to improve the acceptance. 


\section{Muon Track Quality}

Muon track quality is based on number of hits in the SMT or CFT system, $\chi^{2}$ per degrees of freedom for the central track fit, and the distance of closest approach (DCA) with respect to the primary vertex of the event.

- loose track : $|d c a|<0.2 \mathrm{~cm},|d c a|<0.02 \mathrm{~cm}$ if $N_{S M T}>0$

- medium track : loose track $+\chi^{2} / \operatorname{dof}<4$

- tight track : medium track $+N_{S M T}>0$

The medium track quality was used in this analysis.

\section{Muon Isolation}

Muon isolation is based on five variables:

- TrackHalo $=\mid \sum_{\text {tracks }} p_{T}$ in $\Delta R($ track, $\mu)<0.5$ cone

- CalorimeterHalo $=\left|\sum_{\text {cells }} E_{T}\right|$ in hollow cone of $0.1<\Delta R($ cell, track $)<0.4$

- $\Delta R(\mu, j e t)$ distance to closest jet

- ScaledTrackHalo $=$ TrackHalo $/ p_{T}(\mu)$

- ScaledCalorimeterHalo $=$ CalorimeterHalo $/ p_{T}(\mu)$

A cut of ScaledTrackHalo $<0.15$ and ScaledCalorimeterHalo $<0.15$ was used in this analysis.

\subsection{Missing Transverse Energy Reconstruction}

The momentum of the proton and anti-proton beams at the Tevatron are directed precisely along the $z$ axis. This means that there is no momentum transverse to the beam. Missing transverse momentum then is the vector momentum in the transverse plane that must be 
added to the observed event to ensure that the total momentum in the transverse plane is zero. Written out explicitly this is:

$$
\overrightarrow{p_{T}}=-\sum \vec{p}_{T}
$$

For historical reasons the quantity is routinely referred to as missing transverse energy rather than momentum, in a hadron collider you measure the vector sum of visible momenta by taking the energy in each cell of the calorimeter and assigning it a direction based on the position of the cell.

\subsubsection{Missing Transverse Momentum Corrections}

The missing transverse energy is corrected for several important effects:

- EM energy scale, JES correction, to correct the raw $\not p_{T}$ for calibrations applied to electrons and jets.

- bad jets, these are reconstructed jets which fail the jet identification cuts, and are not EM objects.

- muon calorimeter correction, remove energy from the $\not \not_{T}$ that is connected to passage of muons through the calorimeter.

- muon momentum, muons aren't included in the default $\not p_{T}^{\prime}$, they should be added if they come from the hard scatter.

\subsubsection{Missing Transverse Momentum Resolution, $\not q_{T}$ Significance.}

The resolution of the $\not_{T}$ will be affected by the resolution of the objects in the event, the electrons, jets and muons. In addition, there are random fluctuations in the calorimeter energy, such that the background resolution of the calorimeter rises linearly with $\sqrt{\sum E_{\text {unclustered }}}$, in fig. 3.9 this is measured in $Z \rightarrow e e$ data and Monte Carlo.

Recognizing these contributions, we can construct an algorithm to distinguish events with a large $\not_{T}$ due to the presence of neutrinos from those events whose $\not p_{T}$ is due entirely 
to the resolution of the objects in them. This is the MissingPT significance algorithm. It begins by adding in quadrature the resolutions from all of the objects in the event (electrons, muons, jets), projected in the direction of the $\vec{p}_{T}$, with the fluctuations of the unclustered energy in the calorimeter $\left(\sigma_{U E}\right)$ :

$$
\sigma=\sqrt{\sum_{i=e, j}\left[\sigma_{i}\left(E_{i}\right) \cos \Delta \phi_{i}\right]^{2}+\sum_{\mu}\left[p_{T \mu}^{2} \sigma_{\mu}\left(p_{T \mu}^{-1}\right) \cos \Delta \phi_{\mu}\right]^{2}+\sigma_{U E}^{2}} .
$$

The significance is then defined as:

$$
p_{T}^{\sigma}=\log \frac{\operatorname{Gaus}\left(\not p_{T}, \not p_{T}, \sigma\right)}{\operatorname{Gaus}\left(0, \not p_{T}, \sigma\right)}=\frac{p_{T}^{2}}{2 \sigma^{2}}
$$

Where $\operatorname{Gaus}(x, \bar{x}, \sigma)=\frac{1}{\sigma \sqrt{2 \pi}} \exp \frac{(x-\bar{x})^{2}}{2 \sigma^{2}}$.

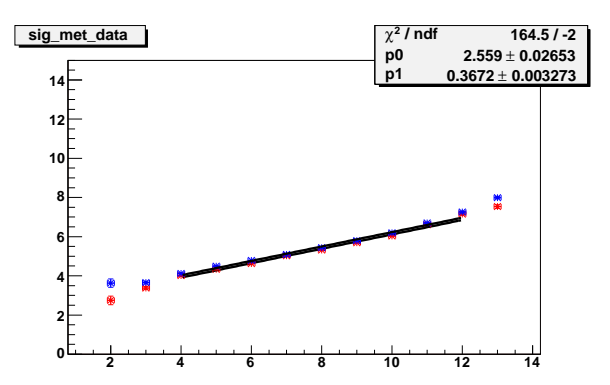

Figure 3.9: Resolution of $\not p_{T}$ as a function of $\sqrt{U E}$ in data (red) and Monte Carlo (blue). 


\section{Chapter 4}

\section{DØ Data}

\subsection{Time Periods}

DØ was conceived of in 1983, and construction on the original detector was completed in 1992. The experiment took $\sim 100 \mathrm{pb}^{-1}$ of data from 1992 to 1996 , a period referred to as Run I. Then from 1996 to 2001 a series of upgrades to the detector were installed. The experiment is now in Run II, and has been taking data since 2001. In 2006 a series of upgrades were made, both to the experiment and the accelerator. The period before the upgrades is referred to as Run IIa, the period after as Run IIb. The upgrades to the accelerator have led to higher instantaneous luminosities, so while Run IIa collected $1 \mathrm{fb}^{-1}$

of usable data in 5 years, Run IIb has collected $4 \mathrm{fb}^{-1}$ in the 3 years since the upgrade.

The running of the Tevatron is broken up into stores. Each store begins when protons and antiprotons are injected into the Tevatron ring and accelerated to an energy of $960 \mathrm{GeV}$. Within each store, DØ breaks up data taking into runs, where each run represents $2-4$ hours of data taking. Each run is broken up into luminosity blocks, where each luminosity block is a few seconds long, and represents a period of constant instantaneous luminosity, and any sudden changes in the detector, or in the luminosity of the collider will automatically lead to the starting of a new luminosity block. 


\subsection{Data Quality}

Some of the data will be affected by problems in the detector subsystems. The worst of these require whole runs to be declared unusable, less serious problems will require single luminosity blocks be removed. These problems include all manner of hardware, electronics, and occasionally accelerator failures. The least serious require individual events be removed.

Some of the most important data quality issues come from the calorimeter. Hardware failures or changes in pedestal can cause individual cells, towers, or whole BLS boards to produce abnormally large signals, or disappear entirely. External noise can be introduced, frequently affecting all the cells attached to one high voltage power supply. Coherent noise can be an issue, particularly when it comes and goes intermittently. These effect necessitate careful data quality monitoring both during and after data taking. 


\section{Chapter 5}

\section{Monte Carlo}

\subsection{Event Generators: ALPGEN, PYTHIA}

We use ALPGEN [33] to simulate the $t \bar{t}$ and $Z+j e t s$ processes. ALPGEN is a parton level matrix-element generator. It performs a numerical evaluation of the S-matrix, without making explicit reference to Feynman diagrams. Avoiding the Feynman diagrams makes it possible to perform parton level calculations involving large numbers of final state partons. The output of ALPGEN is then fed into the PYTHIA [34] event generator, which showers parton-level quarks and gluons into hadrons. The combination of ALPGEN and PYTHIA was used rather than PYTHIA alone, as it was found to better describe high jet multiplicity events.

\subsubsection{Parton Jet Matching, Heavy Flavor Removal}

Combining ALPGEN and PYTHIA produces a potential problem. ALPGEN produces hard partons down to some lower cutoff scale, while PYTHIA produces parton showers starting at some upper cutoff scale. To handle the overlap between ALPGEN and PYTHIA, we use the MLM prescription [35], to match parton showers to the matrix element. The MLM prescription uses an exclusive matching between the partons produced by ALPGEN, and particle jets formed at the end of the parton shower performed by PYTHIA. Matching here simply means that the parton and particle jet must have a $\Delta R$ less than some value. 
In practice this involves generating ALPGEN samples with 0, 1, 2, or more than 3 partons, and requiring that each parton be matched to one and only one particle jet for the 0,1 , and 2 parton samples, and requiring that each parton be matched to at least one particle jet for the 3 parton sample.

Heavy flavor $(b, c)$ quarks can be generated in two ways: the first is through the PYTHIA parton shower, the second is explicitly by the ALPGEN event generator. To avoid double counting when combining ALPGEN samples generated without heavy flavor partons and those generated with heavy flavor partons, a heavy flavor removal scheme is used. For $Z+$ jets the scheme first removes all $c \bar{c}$ and $b \bar{b}$ partons produced by the PYTHIA shower from the light flavor ALPGEN sample. Next all the $c \bar{c}$ partons produced by PYTHIA in the ALPGEN $Z+b \bar{b}$ sample. The last sample, $Z+c \bar{c}$ is left alone [36].

\subsection{Monte Carlo Production Chain}

To produce a usable sample of simulated events, say $t \bar{t} \rightarrow e^{+} \nu_{e} b+\mu^{-} \bar{\nu}_{\mu} \bar{b}$, we start with the matrix element event generator ALPGEN. As described in the previous section, PYTHIA is used to shower the patrons produced by ALPGEN into a set of final state particles. Specialized programs are used to simulate the decay of taus (TAUOLA [37] [38]), the decay of B-hadrons (EVTGEN [39]), and QED radiative corrections (PHOTOS [40] [41]). Next the interaction of the final state particles with the material in the detector is simulated using DØ's version of GEANT (GEometry ANd Tracking [42]). Then the program DØSim is used to: add minimum bias (data events recorded with beam but without trigger bias) into the hard scatter events, add calorimeter pileup for previous events, add calorimeter noise, and add noise and inefficiencies for the SMT, CFT and Muon systems. Finally the standard reconstruction program (DØReco), is used to reconstruct final state objects.

Monte Carlo simulations for the diboson processes are generated with PYTHIA as the event generator, while the $t \bar{t}$ and $Z j j+Z b b+Z c c$ samples are generated with ALPGEN [33] as the event generator using PYTHIA to shower the generated partons and following the MLM prescription [35] for matching the parton shower to the matrix element. 


\subsection{Monte Carlo Corrections}

We know, from extensive study, that the result of our full Monte Carlo simulation does not perfectly model our data, and we apply a variety of corrections to the Monte Carlo to remove this difference.

\subsection{1 $Z$ Boson Transverse Momentum Reweighting}

The inclusive $Z$ Boson Transverse Momentum has been measured using an unfolding procedure which removes the detector level effects (energy scale and resolution, acceptance) [1]. Comparison between the $Z p_{T}$ spectrum in ALPGEN and PYTHIA, the event generators we use to generate our Monte Carlo, reveals a substantial difference, and a reweighting of the Monte Carlo is performed to match the unfolded data [43].

After applying this inclusive reweighting, it has been found that the $Z$ Boson $p_{T}$ spectrum in events with 1 or more jets is different in data and monte carlo, and an ad-hoc reweighting has been applied to the events with $N_{\text {jet }}=1$ and $N_{\text {jet }} \geq 2$ [12]. Figure 5.1 shows the impact of this reweighting for events with one $20 \mathrm{GeV}$ jet, while Fig. 5.2 shows the impact for events with two or more $20 \mathrm{GeV}$ jets.
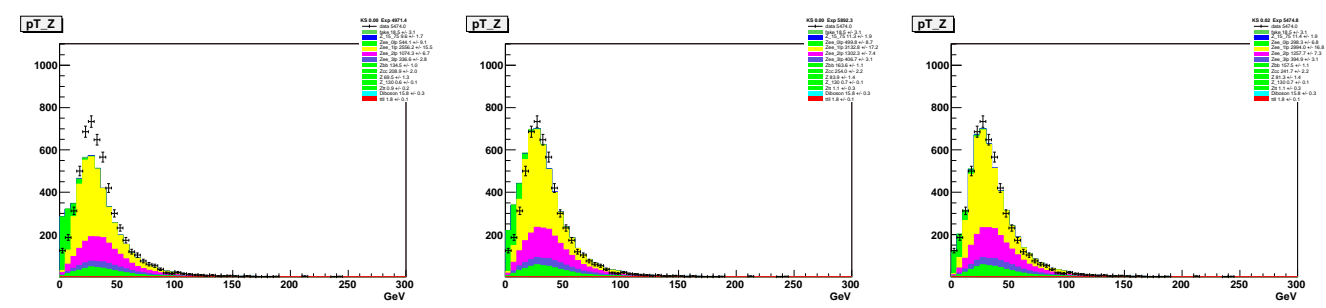

Figure 5.1: Data vs MC for $N_{\text {jet } 20}=1$ for events with no zpt-reweighting, the inclusive reweighting, and the final per-jet reweighting, with Run IIb data/MC [12].

\subsubsection{Beam Longitudinal Position Reweighting}

The Monte Carlo uses a fixed model for the distribution of the $|z|$ position of the Primary Vertex. However, it is known both from the reconstruction of Primary Verticies in events, and the more immediate determination provided by the luminosity monitor(see sec. 2.6), 

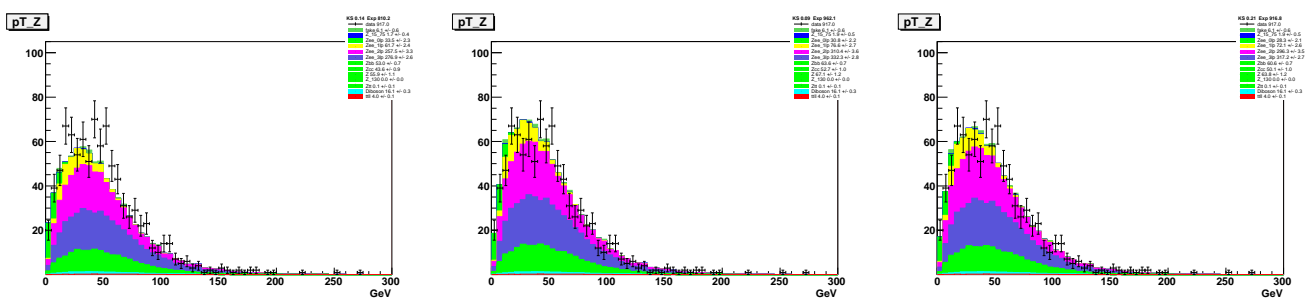

Figure 5.2: Data vs $\mathrm{MC}$ for $N_{\text {jet } 20} \geq 2$ for events with no zpt-reweighting, the inclusive reweighting, and the final per-jet reweighting, with Run IIb data/MC [12].

that the true distribution of the $|z|$ of the Primary Vertex (or the beam spot), is different within different time periods (due to small shifts in the low $\beta$ quadrupole magnets used to focus the $\mathrm{p}$ and $\bar{p}$ beams). This effect has been studied in detail, and weights are applied to the Monte Carlo to match the data [44].

\subsubsection{Instantaneous Luminosity Reweighting}

Underlying event, and multiple interaction effects are not simulated in our Monte Carlo generators (or are not well simulated). To ensure that these effects appear in our simulation, we use minimum bias data, where we require that a beam crossing and collisions have occurred, but do not require any physics trigger. To ensure that our simulation has a luminosity profile matching that in our data, and to therefore ensure especially that multiple interaction effects (which increase linearly with instantaneous luminosity) are accurately reflected in Monte Carlo, we apply weights to the Monte Carlo which give it the same luminosity profile as the data [45], the weight is forced to be less than 3 to avoid having bins with arbitrarily large, or undefined weights.

\subsubsection{Electron and Muon Identification Efficiency Scale Factor}

The efficiency for electrons to pass the electron identification cuts and muons to pass the muon identification cuts has been measured in both data and Monte Carlo. The efficiencies are measured with an identical tag and probe method, using the process $Z \rightarrow e^{+} e^{-}$in the case of electrons and $Z \rightarrow \mu^{+} \mu^{-}$in the case of muons. The tag electron or muon is required to pass tight id cuts, and the invariant mass of the tag and probe is required to be in the 
$Z$ peak. The efficiencies are different in data and Monte Carlo, and weights are applied to the Monte Carlo to correct the difference [46][47].

\subsubsection{Jet Identification Efficiency Correction through Jet Removal}

The efficiency for jets to pass the jet identification requirements is smaller in data than in Monte Carlo. To account for this difference, Monte Carlo jets are removed so that the final efficiency in Monte Carlo matches that in data.

\subsubsection{Electron Energy Smearing}

The electron energy resolution in Monte Carlo is different from that in data. The electron energy in Monte Carlo has been smeared to match that in data [48].

\subsubsection{Muon Momentum Smearing}

The muon momentum is taken entirely from the momentum of the central track. The resolution in Monte Carlo is found to be different from that in data, and the Monte Carlo is smeared to match data (see sec 3.1.3 and [23][24]).

\subsubsection{Jet Smearing, Shifting and Removal}

Jets in all Monte Carlo samples are smeared to match the resolution of jets in data. In addition, a $p_{T}$ dependent shifting factor is applied to jets in all samples except $t \bar{t}$ [32]. The purpose of the shifting is to remove an observed difference between data and Monte Carlo energy scales seen after smearing. 


\begin{tabular}{l|l}
\hline \hline Monte Carlo Sample & $\sigma$ \\
\hline$t \bar{t} m_{t}=172.5 \mathrm{GeV}$ & $7.45 \mathrm{pb}$ \\
$Z \rightarrow l l 15<m_{Z}<75 \mathrm{GeV}$ & $658.0 \mathrm{pb}$ \\
$Z \rightarrow l l 75<m_{Z}<130 \mathrm{GeV}$ & $313.8 \mathrm{pb}$ \\
$Z \rightarrow l l 130<m_{Z}<250 \mathrm{GeV}$ & $2.34 \mathrm{pb}$ \\
$Z \rightarrow l l 250<m_{Z} \mathrm{GeV}$ & $0.26 \mathrm{pb}$ \\
$Z+b \bar{b} \rightarrow l l 15<m_{Z}<75 \mathrm{GeV}$ & $3.16 \mathrm{pb}$ \\
$Z+b \bar{b} \rightarrow l l 75<m_{Z}<130 \mathrm{GeV}$ & $2.57 \mathrm{pb}$ \\
$Z+b \bar{b} \rightarrow l l 130<m_{Z}<250 \mathrm{GeV}$ & $0.02 \mathrm{pb}$ \\
$Z+b \bar{b} \rightarrow l l 250<m_{Z} \mathrm{GeV}$ & $0.002 \mathrm{pb}$ \\
$Z+c \bar{c} \rightarrow l l 15<m_{Z}<75 \mathrm{GeV}$ & $26.05 \mathrm{pb}$ \\
$Z+c \bar{c} \rightarrow l l 75<m_{Z}<130 \mathrm{GeV}$ & $8.03 \mathrm{pb}$ \\
$Z+c \bar{c} \rightarrow l l 130<m_{Z}<250 \mathrm{GeV}$ & $0.065 \mathrm{pb}$ \\
$Z+c \bar{c} \rightarrow l l 250<m_{Z} \mathrm{GeV}$ & $0.0065 \mathrm{pb}$ \\
$W W$ & $11.624 \mathrm{pb}$ \\
$W Z$ & $3.254 \mathrm{pb}$ \\
$Z Z$ & $1.334 \mathrm{pb}$ \\
\hline \hline
\end{tabular}

Table 5.1: Cross Sections for Monte Carlo samples. 


\section{Chapter 6}

\section{Event Selection}

\subsection{Selections for Analysis}

The analysis was performed for two different time periods, Run IIa with $1 \mathrm{fb}^{-1}$ of integrated luminosity, and Run IIb with $3 \mathrm{fb}^{-1}$. The Run IIa analysis used five channels: $e \mu, e e, \mu \mu$, $e+$ track, and $\mu+$ track. The Run IIb analysis used only the three fully reconstructed channels: $e \mu, e e, \mu \mu$.

\subsection{1 $e \mu$ channel selection}

In the $e \mu$ channel the largest backgrounds are: events with one or more fake leptons, $Z \rightarrow \tau \tau+j e t s$, and $W W+$ jets. Fake events can be suppressed using lepton quality cuts. The other main backgrounds are suppressed with a cut to $H_{T}^{l}=\max \left(p_{T}^{e}, p_{T}^{\mu}\right)+p_{T}^{j e t 1}+p_{T}^{j e t 2}$. This cut takes advantage of the soft jets jets in $Z \rightarrow \tau \tau$ and $W W$, and the soft leptons in $Z \rightarrow \tau \tau$. A summary of the selection used is given below, the efficiency of the selection for a $t \bar{t} \rightarrow l l$ sample is given in table 6.1 , a summary of event yields is given in table 6.2 , and plots of data versus simulation are given in Appendix C :

- 1 Top_tight electron with $p_{T}>15 \mathrm{GeV}$ and $|\eta|<1.1$ or $1.5<|\eta|<2.5$

$\bullet \geq 1$ muon with $p_{T}>15 \mathrm{GeV}$ and $\left|\eta_{\text {det }}\right|<2.0$ satisfying:

- Loose quality (Run IIa used MediumNseg3) 
- TopScaledMedium isolation [47]:

* TrackHalo $/ p_{T}^{\mu}<0.15$

* CalorimeterHalo $/ p_{T}^{\mu}<0.15$

- Medium track quality

- No common track between a muon and an electron

- require the electron and muon to have opposite sign

- $\geq 2$ jets with $p_{T}>20 \mathrm{GeV}$

- $H_{T}^{l}>105 \mathrm{GeV}$ (Run IIa used $H_{T}^{l}>115 \mathrm{GeV}$ )

We use all available triggers in the $e \mu$ channel. There is a possible bias in the $Z \rightarrow \tau \tau$ yield of $5 \%$ (arising from the soft lepton spectrum in such events [49]). Given that the statistical error on the $Z \tau \tau$ yield in our final sample is $7 \%$, and that the effect on $t \bar{t}$ and $W W$ samples is less than $2 \%$, we have not accounted specifically for this effect, but expect its impact on the final analysis to be negligible.

\subsection{2 ee channel selection}

In the ee channel the dominant background is $Z \rightarrow e e+j e t s$, this background is suppressed by vetoing events in the $Z$ peak, and cutting on $\not p_{T}$. A summary of the cuts is given below:

- 2 Top_tight electrons with $p_{T}>15 \mathrm{GeV}$ and $|\eta|<1.1$ or $1.5<|\eta|<2.5$ (Top_loose electrons used for fake rate determination)

- Veto Loose quality, TopScaledMedium isolated muons with Medium quality tracks.

- Require electrons have opposite sign and $m_{e e}>15 \mathrm{GeV}$.

- Two jets with $p_{T}>20 \mathrm{GeV}$

- Veto events with $80<m_{e e}<100 \mathrm{GeV}$

- Final Selection: 


\begin{tabular}{l|c|c}
\hline Cut / Scale Factor & Cumulative Efficiency & Data/MC Scale Factor \\
\hline Beam $z$ Position Reweighting & & $99.95 \pm 0.17 \%$ \\
Instantaneous luminosity reweighting & & $99.55 \pm 0.14 \%$ \\
b Hadron Fragmentation Reweighting & & $98.30 \pm 0.19 \%$ \\
DQ Bad Runs/LBNs & $99.87 \pm 0.08 \%$ & \\
DQ Event Based & $96.67 \pm 0.08 \%$ & \\
$|Z|$ of Primary Vertex <60cm & $93.70 \pm 0.08 \%$ & \\
Number of Tracks for Primary Vertex & $92.53 \pm 0.08 \%$ & $98.61 \pm 0.01 \%$ \\
$=1$ Top_loose electrons & $31.47 \pm 0.05 \%$ & $96.03 \pm 0.04 \%$ \\
EMid scale factor - preselection & & $99.30 \pm 0.04 \%$ \\
EMid scale factor & $8.96 \pm 0.02 \%$ & $91.02 \pm 0.04 \%$ \\
$\geq 1$ loose muon & & \\
MuonID quality scale factor & & \\
MuonID track quality scale factor & & \\
MuonID isolation scale factor & $7.86 \pm 0.02 \%$ & \\
No common track between e and $\mu$ & $7.86 \pm 0.02 \%$ & \\
$\leq 1$ electron & $7.75 \pm 0.02 \%$ & \\
require opposite sign e $\mu$ pair & $7.74 \pm 0.02 \%$ & \\
$\Delta R_{e} \geq 0.3$ & $5.99 \pm 0.02 \%$ & \\
$\geq 2$ jets $p_{T}>20$ GeV & $5.76 \pm 0.02 \%$ & \\
$H_{T}^{l} \geq 105$ GeV & & \\
Likelihood $\geq 0.85$ correction & & \\
Likelihood $\geq 0.85$ & $4.82 \pm 0.02 \%$ & \\
\hline
\end{tabular}

Table 6.1: Cumulative efficiency and data over Monte Carlo scale factors for $t \bar{t} \rightarrow l \bar{l} \nu \bar{\nu} b \bar{b}$ signal with $m_{t}=172.5 \mathrm{GeV}$ in $e \mu$ channel in Run IIb.

\begin{tabular}{l|c|c|c|c}
\hline & inclusive & $\geq 1$ jet & $\geq 2$ jets & $H_{T}^{l}>105 \mathrm{GeV}$ \\
\hline$Z \rightarrow \tau \tau$ & $1585 \pm 22.5$ & $271.4 \pm 8.3$ & $37.4 \pm 2.2$ & $19.6 \pm 1.5$ \\
$W W / W Z / Z Z$ & $254.8 \pm 3.6$ & $51 \pm 1.6$ & $10.1 \pm 0.7$ & $7.9 \pm 0.6$ \\
fake & $202.2 \pm 3.6$ & $72.7 \pm 2.4$ & $20.7 \pm 0.9$ & $14 \pm 0.7$ \\
$t \bar{t} \rightarrow l \bar{l}$ & $154.5 \pm 0.5$ & $152.6 \pm 0.5$ & $120.2 \pm 0.5$ & $115.5 \pm 0.6$ \\
\hline total & $2196.5 \pm 23.1$ & $547.7 \pm 8.8$ & $188.4 \pm 2.6$ & $157 \pm 1.8$ \\
\hline data & 2131 & 601 & 201 & 173 \\
\hline
\end{tabular}

Table 6.2: Yield for $e \mu$ selection in Run IIb. Note: all selections have $L_{e} \geq 0.85$ requirement. 
$-\not p_{T}>40 \mathrm{GeV}$

- When $m_{e e}<80 \mathrm{GeV}$ require $\not{ }_{T}>45 \mathrm{GeV}$

We require that events pass one of the single electron triggers or one of the dielectron triggers. The efficiency of this OR of triggers is very high for the Top_tight electron definition, the efficiency of the single electron triggers alone is $>99 \%$ for $t \bar{t} \rightarrow e e$ events, and we take the efficiency of the OR with dielectron triggers to be $100 \%$.

Table 6.3 gives the signal efficiency for the $t \bar{t} \rightarrow l \bar{l} \nu \bar{\nu} b \bar{b}$ signal, while table 6.4 gives the selection yields.

\begin{tabular}{|c|c|c|}
\hline Cut / Scale Factor & Cumulative Efficiency & Data/MC Scale Factor \\
\hline Beam $z$ Position Reweighting & & $99.95 \pm 0.17 \%$ \\
\hline Instantaneous luminosity reweighting & & $99.55 \pm 0.14 \%$ \\
\hline b Hadron Fragmentation Reweighting & & $98.30 \pm 0.19 \%$ \\
\hline DQ Bad Runs/LBNs & $99.86 \pm 0.08 \%$ & \\
\hline DQ Event Based & $96.68 \pm 0.08 \%$ & \\
\hline$|Z|$ of Primary Vertex $<60 \mathrm{~cm}$ & $93.66 \pm 0.08 \%$ & \\
\hline Number of Tracks for Primary Vertex & $92.48 \pm 0.08 \%$ & \\
\hline$\geq 2$ Top_loose electrons & $4.60 \pm 0.02 \%$ & \\
\hline$\leq 2$ Top_tight electron & $4.59 \pm 0.02 \%$ & \\
\hline veto tight muon & $4.48 \pm 0.02 \%$ & \\
\hline require opposite sign ee pair & $4.30 \pm 0.02 \%$ & \\
\hline EMid scale factor - preselection & & $97.23 \pm 0.02 \%$ \\
\hline EMid scale factor & & $77.63 \pm 0.15 \%$ \\
\hline 2 Top_tight electrons & $2.37 \pm 0.01 \%$ & \\
\hline$\geq 2$ jets $p_{T}>20 \mathrm{GeV}$ & $1.75 \pm 0.01 \%$ & \\
\hline veto $80<m_{e e}<100 \mathrm{GeV}$ & $1.49 \pm 0.01 \%$ & \\
\hline$\not p_{T}>45 \mathrm{GeV}$ for $m_{e e}<80 \mathrm{GeV}$ & $1.23 \pm 0.01 \%$ & \\
\hline$\not \not_{T}>40 \mathrm{GeV}$ for $m_{e e}>100 \mathrm{GeV}$ & $1.03 \pm 0.01 \%$ & \\
\hline
\end{tabular}

Table 6.3: Cut flow efficiency for $t \bar{t} \rightarrow l \bar{l} \nu \bar{\nu} b \bar{b}$ signal with $m_{t}=172.5 \mathrm{GeV}$ in $e e$ channel in Run IIb.

\subsection{3 $\mu \mu$ channel selection}

In the $\mu \mu$ channel the dominant background is $Z \rightarrow \mu \mu+$ jets. The poor resolution of the muon reduces the usefulness of cuts on $m_{\mu \mu}$ or directly on the $\not q_{T}$. To make up for this we use the $\not p_{T}^{\prime}$ - significance algorithm (see section 3.5.2). 


\begin{tabular}{l|c|c|c|c|c}
\hline & inclusive & $\geq 1$ jet & $\geq 2$ jets & $\begin{array}{c}\text { Z veto } \\
\not ⿰ T\end{array}$ & $\begin{array}{c}\text { final } \\
\text { selection }\end{array}$ \\
\hline$Z \rightarrow e e$ & $123235 \pm 207$ & $17748 \pm 62$ & $2566.4 \pm 19.6$ & $22.0 \pm 1.9$ & $2.2 \pm 0.3$ \\
$Z \rightarrow \tau \tau$ & $532.8 \pm 12.5$ & $74.0 \pm 4.0$ & $10.7 \pm 1.1$ & $6.4 \pm 0.9$ & $3.2 \pm 0.6$ \\
$W W / W Z / Z Z$ & $179.0 \pm 2.3$ & $88.2 \pm 1.3$ & $41.2 \pm 0.8$ & $1.9 \pm 0.3$ & $1.3 \pm 0.2$ \\
fake & $291.8 \pm 118.5$ & $90.2 \pm 17.2$ & $16.7 \pm 2.7$ & $2.7 \pm 0.1$ & $1.3 \pm 0.1$ \\
$t \bar{t} \rightarrow l \bar{l} \nu \bar{\nu} b \bar{b}$ & $55.7 \pm 0.4$ & $54.7 \pm 0.4$ & $41.4 \pm 0.3$ & $29.3 \pm 0.3$ & $24.4 \pm 0.3$ \\
\hline total expected & $124294 \pm 239$ & $18056 \pm 65$ & $2676.3 \pm 19.8$ & $62.3 \pm 2.1$ & $32.3 \pm 0.7$ \\
\hline data & 116496 & 16630 & 2489 & 49 & 34 \\
\hline
\end{tabular}

Table 6.4: Yield for ee selection in Run IIb.

We cut on $p_{T}^{\sigma}$ to maximize the signal significance $(S / \sqrt{S+B})$. A full summary of the selection is given below:

- 2 muons with $p_{T}>15 \mathrm{GeV}$ and $\left|\eta_{\text {det }}\right|<2.0$ satisfying:

- Loose quality (Run IIa used MediumNseg3)

- TopScaledMedium isolation

- Medium track quality

- veto on Top_tight electrons with $p_{T}>15 \mathrm{GeV}$ and $|\eta|<1.1$ or $1.5<|\eta|<2.5$

- require muons to have opposite sign, also require $m_{\mu \mu}>30 \mathrm{GeV}$

- Two jets with $p_{T}>20 \mathrm{GeV}$

- Final Selection:

$$
\begin{aligned}
& -p_{T}^{\sigma}>7.5 \text { for } m_{\mu \mu}<70 \mathrm{GeV} \\
& -p_{T}^{\sigma}>10.2 \text { for } 70<m_{\mu \mu}<110 \mathrm{GeV} \\
& -p_{T}^{\sigma}>4.2 \text { for } m_{\mu \mu}>110 \mathrm{GeV}
\end{aligned}
$$

We require that the event pass an OR of single muon triggers, the efficiency applied to Monte Carlo is measured using the method described in [50].

Table 6.5 gives the signal efficiency for the $t \bar{t} \rightarrow l \bar{l} \nu \bar{\nu} b \bar{b}$ signal, while table 6.6 gives the selection yields. 


\begin{tabular}{l|c|c}
\hline Cut / Scale Factor & Cumulative Efficiency & Data/MC Scale Factor \\
\hline Beam $z$ Position Reweighting & & $99.98 \pm 0.17 \%$ \\
Instantaneous luminosity reweighting & & $99.99 \pm 0.13 \%$ \\
b Hadron Fragmentation Reweighting & $99.86 \pm 0.08 \%$ & $98.30 \pm 0.19 \%$ \\
DQ Bad Runs/LBNs & $96.68 \pm 0.08 \%$ & \\
DQ Event Based & $93.66 \pm 0.08 \%$ & \\
$|Z|$ of Primary Vertex $<60 \mathrm{~cm}$ & $92.48 \pm 0.08 \%$ & \\
Number of Tracks for Primary Vertex & $61.72 \pm 0.07 \%$ & \\
veto Top_tight electrons & $4.90 \pm 0.02 \%$ & \\
$\geq 2 \mu$ & & $98.61 \pm 0.08 \%$ \\
MuonID quality scale factor & & $93.03 \pm 0.07 \%$ \\
MuonID track quality scale factor & & $80.13 \pm 0.16 \%$ \\
MuonID isolation scale factor & & \\
Single Muon OR Trigger efficiency & & \\
require opposite sign $\mu \mu$ pair $m_{\mu \mu}>30 \mathrm{GeV}$ & $2.86 \pm 0.01 \%$ & \\
$>=2$ jets $p_{T}>20 \mathrm{GeV}$ & $2.31 \pm 0.01 \%$ & \\
$70<m_{\mu \mu}<110 \mathrm{GeV}: p_{T}^{\sigma}>10.2$ & $1.85 \pm 0.09 \%$ & \\
$m_{\mu \mu}<70 \mathrm{GeV}: p_{T}^{\sigma}>7.5$ & $1.51 \pm 0.08 \%$ & \\
$m_{\mu \mu}>110 \mathrm{GeV}: p_{T}^{\sigma}>4.2$ & $1.09 \pm 0.07 \%$ & \\
\hline
\end{tabular}

Table 6.5: Cut flow efficiency for $t \bar{t} \rightarrow l \bar{l} \nu \bar{\nu} b \bar{b}$ signal with $m_{t}=172.5 \mathrm{GeV}$ in $\mu \mu$ channel in Run IIb.

\begin{tabular}{l|c|c|c|c|c}
\hline & inclusive & $\geq 1$ jet & $\geq 2$ jets & $p_{T}^{\sigma}>2$ & final selection \\
\hline$Z \rightarrow \mu \mu$ & $174729 \pm 225$ & $26344 \pm 71$ & $4128.4 \pm 23.0$ & $334.1 \pm 7.7$ & $2.7 \pm 0.6$ \\
$Z \rightarrow \tau \tau$ & $861.1 \pm 14.9$ & $142.4 \pm 5.0$ & $23.5 \pm 1.8$ & $13.2 \pm 1.3$ & $3.3 \pm 0.7$ \\
$W W / W Z / Z Z$ & $242.2 \pm 2.5$ & $121.0 \pm 1.4$ & $60.7 \pm 0.9$ & $6.9 \pm 0.4$ & $2.2 \pm 0.3$ \\
fake & $152.9 \pm 89.0$ & $246.4 \pm 18.6$ & $58.6 \pm 3.8$ & $7.6 \pm 0.4$ & $2.1 \pm 0.1$ \\
$t \bar{t} \rightarrow l \bar{l} \nu \bar{\nu} b \bar{b}$ & $70.7 \pm 0.4$ & $70.0 \pm 0.4$ & $57.4 \pm 0.4$ & $44.3 \pm 0.3$ & $25.6 \pm 0.2$ \\
\hline total expected & $176054 \pm 244$ & $26860 \pm 73$ & $4311.5 \pm 23.3$ & $406.1 \pm 7.8$ & $36.0 \pm 1.0$ \\
\hline data & 167368 & 27386 & 4388 & 371 & 32 \\
\hline
\end{tabular}

Table 6.6: Yield for $\mu \mu$ selection in Run IIb. 


\subsubsection{Yield for Run IIa}

Similar selections were done in the $1 f b^{-1}$ sample for the $e e[51], e \mu[52]$ and $e \mu[53]$ channels, also in Run IIa a selection was performed in the electron plus charged track and muon plus charged track channels[54]. I list the yields for these five channels in table 6.7.

\begin{tabular}{c|c|c|c|c|c|c}
\hline & $e e$ & $e \mu$ & $\mu \mu$ & e+track & $\mu+$ track & combined \\
\hline$Z \rightarrow e e / \mu \mu$ & $1.0 \pm 0.3$ & & $4.1 \pm 0.7$ & $0.3 \pm 0.1$ & $0.6 \pm 0.1$ & $6.0 \pm 0.8$ \\
$Z \rightarrow \tau \tau$ & $1.2 \pm 0.2$ & $4.4 \pm 0.4$ & $1.9 \pm 0.2$ & $0.1 \pm 0.0$ & $0.1 \pm 0.0$ & $7.7 \pm 0.5$ \\
$W W / W Z / Z Z$ & $0.5 \pm 0.1$ & $1.4 \pm 0.1$ & $0.6 \pm 0.1$ & $0.1 \pm 0.0$ & $0.1 \pm 0.0$ & $2.7 \pm 0.2$ \\
fake & $0.6 \pm 0.2$ & $2.6 \pm 0.5$ & $0.2 \pm 0.2$ & $0.4 \pm 0.1$ & $0.0 \pm 0.0$ & $3.8 \pm 0.6$ \\
$t \bar{t} \rightarrow l \bar{l} \nu \bar{\nu} b \bar{b}$ & $11.7 \pm 0.1$ & $35.6 \pm 0.1$ & $9.2 \pm 0.0$ & $9.4 \pm 0.0$ & $4.6 \pm 0.0$ & $70.5 \pm 0.1$ \\
total expected & $15.0 \pm 0.4$ & $44.0 \pm 0.7$ & $15.9 \pm 0.6$ & $10.3 \pm 0.1$ & $5.4 \pm 0.0$ & $90.7 \pm 1.1$ \\
\hline data & 17 & 39 & 12 & 8 & 6 & 82 \\
\hline
\end{tabular}

Table 6.7: Expected and observed event yields for the dilepton channels in Run IIa.

\begin{tabular}{lcccc}
\hline & $e e$ & $e \mu$ & $\mu \mu$ & combined \\
\hline$Z \rightarrow e e / \mu \mu$ & $2.2 \pm 0.3$ & & $2.7 \pm 0.6$ & $4.9 \pm 0.7$ \\
$Z \rightarrow \tau \tau$ & $3.2 \pm 0.6$ & $19.6 \pm 1.5$ & $3.3 \pm 0.7$ & $26.1 \pm 1.8$ \\
$W W / W Z / Z Z$ & $1.3 \pm 0.2$ & $7.9 \pm 0.6$ & $2.2 \pm 0.3$ & $11.4 \pm 0.7$ \\
fake & $1.3 \pm 0.1$ & $14.0 \pm 0.7$ & $2.1 \pm 0.1$ & $17.4 \pm 0.7$ \\
$t \bar{t} \rightarrow l \bar{l} \nu \bar{\nu} b \bar{b}$ & $24.4 \pm 0.3$ & $115.5 \pm 0.6$ & $25.6 \pm 0.2$ & $165.5 \pm 0.7$ \\
\hline total expected & $32.4 \pm 0.7$ & $157.0 \pm 1.77$ & $36.0 \pm 1.0$ & $225.3 \pm 2.2$ \\
\hline data & 34 & 173 & 32 & 239 \\
\hline
\end{tabular}

Table 6.8: Expected and observed event yields for the dilepton channels in Run IIb.

\begin{tabular}{lccccc}
\hline & $e e$ & $e \mu$ & $\mu \mu$ & ltrk & combined \\
\hline$Z \rightarrow e e / \mu \mu$ & $3.2 \pm 0.4$ & & $6.8 \pm 0.9$ & $0.9 \pm 0.1$ & $10.9 \pm 1.0$ \\
$Z \rightarrow \tau \tau$ & $4.4 \pm 0.6$ & $24.0 \pm 1.6$ & $5.2 \pm 0.7$ & $0.2 \pm 0.0$ & $33.8 \pm 1.8$ \\
$W W / W Z / Z Z$ & $1.8 \pm 0.2$ & $9.3 \pm 0.6$ & $2.8 \pm 0.3$ & $0.2 \pm 0.0$ & $14.1 \pm 0.7$ \\
fake & $1.9 \pm 0.2$ & $16.6 \pm 0.9$ & $2.3 \pm 0.2$ & $0.4 \pm 0.1$ & $21.2 \pm 0.9$ \\
\hline total background & $11.3 \pm 0.8$ & $49.9 \pm 1.9$ & $17.1 \pm 1.2$ & $1.7 \pm 0.2$ & $80.0 \pm 2.4$ \\
\hline$t \bar{t} \rightarrow l l$ & $36.1 \pm 0.3$ & $151.1 \pm 0.61$ & $34.8 \pm 0.2$ & $14.0 \pm 0.0$ & $236.0 \pm 0.7$ \\
\hline total expected & $47.4 \pm 0.9$ & $201.0 \pm 2.0$ & $51.9 \pm 1.3$ & $15.7 \pm 0.2$ & $316.0 \pm 2.5$ \\
\hline data & 51 & 212 & 44 & 14 & 321 \\
\hline
\end{tabular}

Table 6.9: Expected and observed event yields for the dilepton channels for full Run II. 


\subsubsection{Determination of fake rate}

To estimate the background from fake leptons we use the 4-way matrix method approach. In this method we need to distinguish two different lepton types. For the the $e \mu$ channel these are the electron and the muon, for the $e e$ and $\mu \mu$ channels these types are the electron or muon with the largest $p_{T}$ and the electron or muon with the second largest $p_{T}$. We then need to define loose and tight cuts for each lepton type. In the $e \mu$ channel the loose cut for the electron is the Top_loose electron quality, while the tight cut is the Top_tight electron quality. The loose cut for the muon requires loose quality, and medium track quality, while the tight cut for muons adds a TopScaledMedium isolation requirement. Next we count the number of events where both leptons pass the loose cut $\left(N_{l l}\right)$, where the first lepton passes the tight cut and the second the loose cut $\left(N_{t l}\right)$, where the the second passes the tight cut and the first the loose cut $\left(N_{l t}\right)$, and finally where both leptons pass the tight cut $\left(N_{t t}\right)$. Then we measure the efficiency for a real lepton, which has passed the loose cuts, to pass tight cuts $\left(\epsilon_{r 1}, \epsilon_{r 2}\right)$, and the efficiency for a fake lepton, which has passed the loose cuts, to pass tight cuts $\left(\epsilon_{f 1}, \epsilon_{f 2}\right)$, see description below for more detail. Finally, we can form a linear equation as follows:

$$
\begin{gathered}
\nu_{i}=\sum_{j} A_{i j} \lambda_{j}^{l l}, \\
\nu=\left(\begin{array}{c}
N_{l l} \\
N_{t l} \\
N_{l t} \\
N_{t t}
\end{array}\right), \\
\lambda^{l l}=\left(\begin{array}{c}
N_{r r} \\
N_{r f} \\
N_{f r} \\
N_{f f}
\end{array}\right),
\end{gathered}
$$




$$
A=\left(\begin{array}{cccc}
1 & 1 & 1 & 1 \\
\epsilon_{r 1} & \epsilon_{r 1} & \epsilon_{f 1} & \epsilon_{f 1} \\
\epsilon_{r 2} & \epsilon_{f 2} & \epsilon_{r 2} & \epsilon_{f 2} \\
\epsilon_{r 1} \epsilon_{r 2} & \epsilon_{r 1} \epsilon_{f 2} & \epsilon_{f 1} \epsilon_{r 2} & \epsilon_{f 1} \epsilon_{f 2}
\end{array}\right)
$$

Here $\nu$ is an array giving the number of events passing our quality cuts. The array $\lambda^{l l}$ gives the number of events where both leptons are real, the first lepton is real while the second is fake, the second is real while the first is fake, and where both leptons are fake. The matrix $A$ connects the measured yields to the true yields. We are interested in the number of events in which at least one lepton is fake, and we can extract this by first inverting equation 6.4.

$$
\lambda_{i}^{l l}=\sum_{j} A_{i j}^{-1} \nu_{j}
$$

This would allow us to get the number of fake events in the loose sample, but we really want the number in the tight sample. We introduce the matrix $B$ (just the last row of $A$ ) to represent the transformation:

$$
B=\left(\epsilon_{r 1} \epsilon_{r 2}, \epsilon_{r 1} \epsilon_{f 2}, \epsilon_{f 1} \epsilon_{r 2}, \epsilon_{f 1} \epsilon_{f 2}\right)
$$

The final expression is then:

$$
\lambda_{i}^{t t}=B_{i} \lambda_{i}^{l l}=B_{i} \sum_{j} A_{i j}^{-1} \nu_{j}
$$

It is a straightforward exercise to determine the errors:

$$
\left(\delta \lambda_{i}^{l l}\right)^{2}=\sum_{\epsilon}\left[\sum_{j}\left(\frac{\partial B_{i}}{\partial \epsilon} A_{i j}^{-1} \nu_{j}-\sum_{k l} B_{i} A_{i k}^{-1} \frac{\partial A_{k l}}{\partial \epsilon} A_{l j}^{-1} \nu_{j}\right) \delta \epsilon\right]^{2}+\sum_{j}\left(B_{i} A_{i j}^{-1} \delta \nu_{j}\right)^{2} .
$$

To determine $\epsilon_{r}^{e}$, we use a tag and probe method in ee data. One electron is required to be tight, and the efficiency for the other to pass the tight cut is then measured. After requiring $80<m_{e e}<100 \mathrm{GeV}$, a value of $\epsilon_{\text {real }}^{e}=0.8576 \pm 0.0027$ is obtained. 
To determine $\epsilon_{f}^{e}$ we use a tag and probe method in $e \mu$ data, the muon is required to be non-isolated, with both ScaledCalorimeterHalo $>0.2$ and ScaledTrackHalo $>0.2$ and the electron and muon are required to have the same charge. After requiring $\not \not_{T}<20 \mathrm{GeV}$, a value of $\epsilon_{f}^{e}=0.0862 \pm 0.0038$ is obtained.

We use the electron fake rate obtained in the same sign $e \mu$ sample for both the $e e$ and $e \mu$ samples. We do not use the same sign ee sample to determine the fake rate due to the large contamination from real electrons in the tight sample, most of these events have at least one electron in the EC where there is a greater likelihood to misidentify the charge of the track.

To determine $\epsilon_{\text {real }}^{\mu}$, we use a tag and probe method in $\mu \mu$ data, one muon is required to be tight, and the efficiency for the other to pass the tight cut is then measured. After requiring $80<m_{\mu \mu}<100 \mathrm{GeV}$, a value of $\epsilon_{\text {real }}^{e}=0.9690 \pm 0.0029$ is obtained.

To determine $\epsilon_{\text {fake }}^{\mu}$ we use a tag and probe method in $\mu \mu$ data, one muon is required to be non-isolated, with both ScaledCalorimeterHalo $>0.2$ and ScaledTrackHalo $>0.2$ and the muons are required to have the same charge. After requiring $\not_{T}^{\prime}<20 \mathrm{GeV}$, a value of $\epsilon_{\text {fake }}^{\mu}=0.0437 \pm 0.0044$ is obtained.
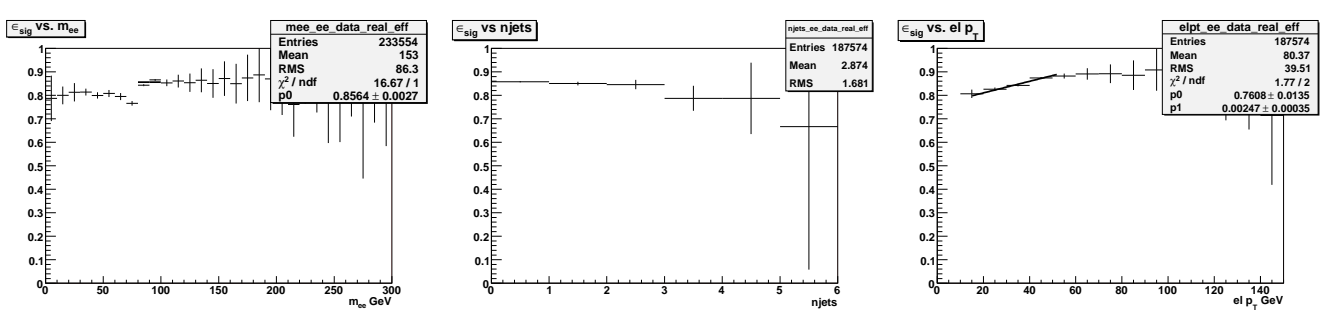

Figure 6.1: $\epsilon_{\text {real }}^{e}$ determination from $Z \rightarrow e e$, versus $m_{e e}$, dependence on $N_{j e t s}, p_{T}^{e}$.
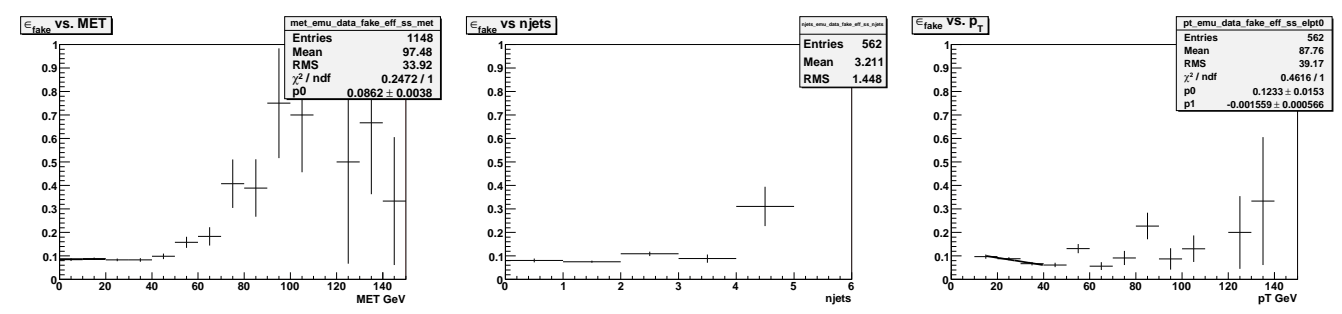

Figure 6.2: $\epsilon_{\text {fake }}^{e}$ determination from anti-isolated $e \mu$ sample, versus $\not \not_{T}$, dependence on $N_{\text {jets }}, p_{T}^{e}$. 

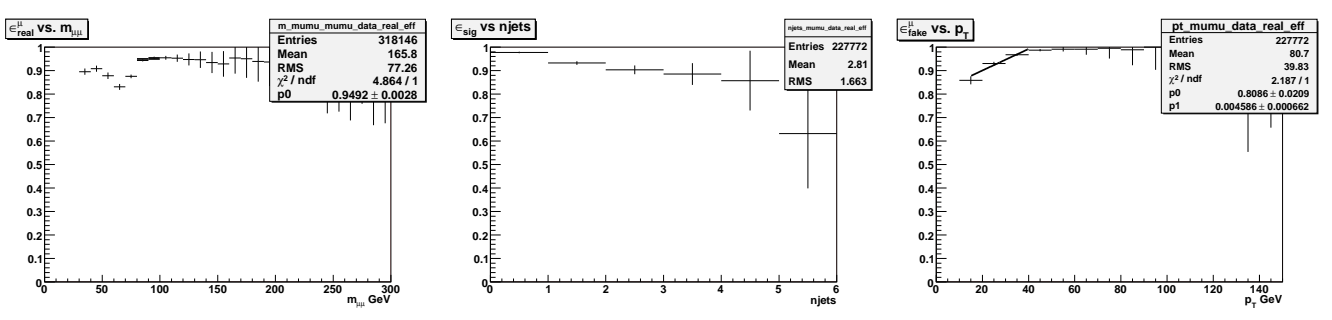

Figure 6.3: $\epsilon_{\text {real }}^{\mu}$ determination from $Z \rightarrow \mu \mu$, versus $m_{\mu \mu}$, dependence on $N_{\text {jets }}, p_{T}^{\mu}$.
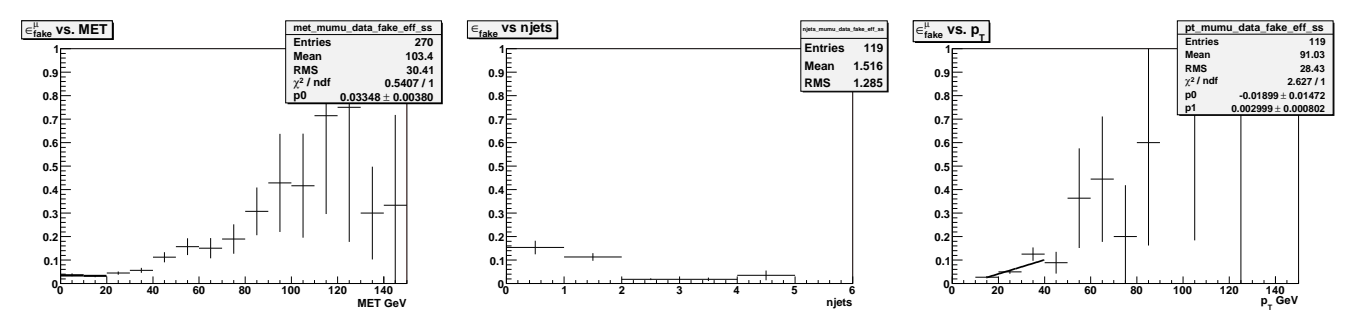

Figure 6.4: $\epsilon_{\text {fake }}^{\mu}$ determination from anti-isolated $\mu \mu$ sample, versus $\not \not_{T}$, dependence on $N_{\text {jets }}, p_{T}^{\mu}$. 


\section{Chapter 7}

\section{Mass Measurement}

\subsection{Kinematic Reconstruction}

In the dilepton channel, there are six final state objects, two leptons, two neutrinos, and two b-jets. The two neutrinos are only observed through the missing transverse momentum in the event. We can then construct a system of eight equations:

$$
\begin{aligned}
\vec{\nu}_{x}+\vec{\nu}_{x} & =\vec{E}_{T, x}, \\
\vec{\nu}_{y}+\vec{\nu}_{y} & =\vec{E}_{T, y}, \\
E_{\nu}^{2}-\vec{p}_{\nu}^{2} & =0, \\
E_{\bar{\nu}}^{2}-\vec{p}_{\bar{\nu}}^{2} & =0, \\
\left(E_{l^{+}}+E_{\nu}\right)^{2}-\left(\vec{p}_{l^{+}}+\vec{p}_{\nu}\right)^{2} & =m_{W^{+}}^{2}, \\
\left(E_{l^{-}}+E_{\bar{\nu}}\right)^{2}-\left(\vec{p}_{l^{-}}+\vec{p}_{\bar{\nu}}\right)^{2} & =m_{W^{-}}^{2}, \\
\left(E_{l^{+}}+E_{\nu}+E_{b}\right)^{2}-\left(\vec{p}_{l^{+}}+\vec{p}_{\nu}+\vec{p}_{b}\right)^{2} & =m_{t}^{2}, \\
\left(E_{l^{-}}+E_{\bar{\nu}}+E_{\bar{b}}\right)^{2}-\left(\vec{p}_{l^{-}}+\vec{p}_{\bar{\nu}}+\vec{p}_{\bar{b}}\right)^{2} & =m_{\bar{t}}^{2} .
\end{aligned}
$$

This system of equations can be fully solved for $p_{z}$ of the two neutrinos, once we specify values for $m_{W^{+}}, m_{W^{-}}, m_{t}$ and $m_{\bar{t}}$. The system of equations can be reduced to a fourth order polynomial, which can be solved analytically to produce at most 4 solutions (see [55][56]). 


\section{$7.2 \quad$ Weighting}

We assign a weight to each solution given by [57]:

$$
w=f(x) f(\bar{x}) p\left(E_{\ell}^{*} \mid m_{t}\right) p\left(E_{\bar{\ell}}^{*} \mid m_{t}\right),
$$

where $f(x)$ is the Parton Distribution Function (PDF) for the proton for the momentum fraction $x$ carried by the initial quark, and $f(\bar{x})$ is the corresponding value for the initial antiquark. The quantity $p\left(E_{\ell}^{*} \mid m_{t}\right)$ is the probability for the hypothesized top quark with mass $m_{t}$ to decay into the lepton $\ell$ with the observed energy $\left(E_{\ell}^{*}\right)$ in the top quark rest frame [57], it is given by:

$$
p\left(E_{\ell}^{*} \mid m_{t}\right)=\frac{4 E_{\ell}^{*} m_{t}\left(m_{t}^{2}-m_{b}^{2}-2 E_{\ell}^{*} m_{t}\right)}{\left(m_{t}^{2}-m_{b}^{2}\right)^{2}-m_{W}^{2}\left(m_{t}^{2}+m_{b}^{2}\right)-2 m_{W}^{4}} .
$$

There are two ways to assign the two jets to the $b$ and $\bar{b}$ quarks. For each assignment of observed momenta to the final state particles, there may be up to four solutions for each hypothesized value of the top quark mass. The likelihood for each value of the top quark mass $m_{t}$ is then given by the sum of the weights over all the possible solutions:

$$
W_{0}\left(m_{t}\right)=\sum_{\text {sol assign }} \sum_{\text {sol,assign }}
$$

\subsection{Smearing of Object Momenta}

The kinematic reconstruction is intended to be used with parton level momenta, which are not the same as the momenta we measure in the detector. We already calibrate our measured momenta to remove constant offsets and differences in scale, but there is an irreducible resolution to our measured momenta.

To account for the resolution of our measured objects, we smear the object momenta according to the distribution of such momenta consistent with the known resolution of the detector.

For jets the momenta are drawn from a double-gaussian distribution, which has been tuned to reflect the relationship between observed jets and partons in Monte Carlo [58] 
(see Appendix B). For electrons we use an $\eta$ dependent parametrization of the energy resolution (see section 3.2.3). For muons the inverse momenta are drawn from a gaussian distribution with an $\eta$ dependent width (see section 3.1.3). The $\not p_{T}$ is corrected by the vector sum of the differences in the particle momenta from the measured values and an added random noise vector with $x$ and $y$-components drawn from a normal distribution with a mean of zero and an RMS which is a function of the total unclustered transverse energy in the event (see figure 3.9).

The smearing is repeated $N=100$ times, and we sum over the weights for each smearing:

$$
W\left(m_{t}\right)=\frac{1}{N} \sum_{n=1}^{N} \sum_{\text {sol }} \sum_{\text {assign }} w_{n, \text { sol,assign }}
$$

\subsection{Template Fit for Top Mass}

For each event we use the value of the hypothesized top quark mass at which $W\left(m_{t}\right)$ reaches its maximum as the estimator for the mass of the top quark. We call this mass value the peak mass $\left(m_{t}^{\max }\right)$. We cannot determine the top quark mass directly from the distribution of peak masses, because effects such as initial and final state radiation shift the most probable value of the peak mass distribution away from the actual top quark mass. We therefore generate the expected distributions of weight curve peaks for a range of top quark masses using Monte Carlo simulations. We call these distributions templates. Figures 7.3 - 7.5 show templates for the signal, while Figures 7.1 and 7.2 show templates for the background.

To extract the mass we use a binned maximum likelihood fit with the following definition for the likelihood:

$$
\mathcal{L}\left(m_{t}\right)=\prod_{i=1}^{n_{\text {bin }}}\left[\frac{n_{s} s_{i}\left(m_{t}\right)+n_{b} b_{i}}{n_{s}+n_{b}}\right]^{n_{i}} .
$$

Here $n_{i}$ is the number of data events observed in bin $i, s_{i}\left(m_{t}\right)$ is the normalized signal template for bin $i$ with top mass $m_{t}, b_{i}$ is the normalized background template, and $n_{s}$ and $n_{b}$ are the number of expected signal and background events. The number of background 
events is taken from tables $6.2,6.4$, and 6.6. The number of signal events is fixed to the number of events expected for a mass of $172.5 \mathrm{GeV}$ and a cross section of $7.45 \mathrm{pb}$, which are also taken from tables $6.2,6.4$, and 6.6 .

In the combined result, we add together the $-\log \mathcal{L}$ 's from the three channels. Finally, we fit a quadratic function to the $-\log \mathcal{L}$ points, fitting over a range of $\pm 20 \mathrm{GeV}$ around the mass with the lowest value of $-\log \mathcal{L}$.
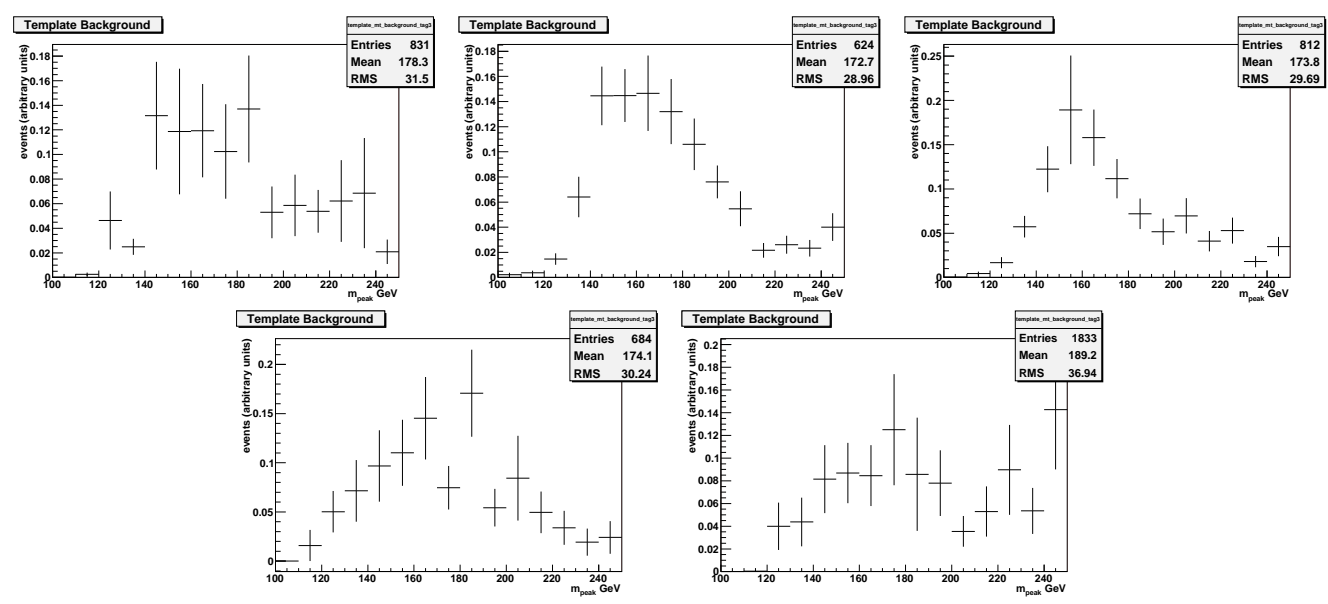

Figure 7.1: Combined background templates for $e e, e \mu, \mu \mu$, e+track and $\mu+$ track in Run IIa.
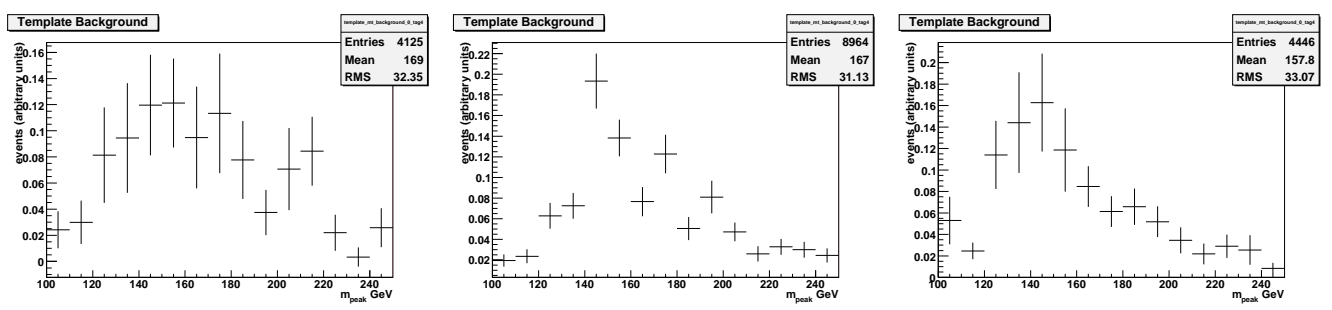

Figure 7.2: Background templates for $e e, e \mu$, and $\mu \mu$ in Run IIb.

\subsection{Performance of Method on Simulated Data}

In order to evaluate the performance of our method, we generate a large number of simulated experiments for several input top quark mass values. We refer to each of these experiments as an ensemble. We fit each of the ensembles to the templates as for collider 

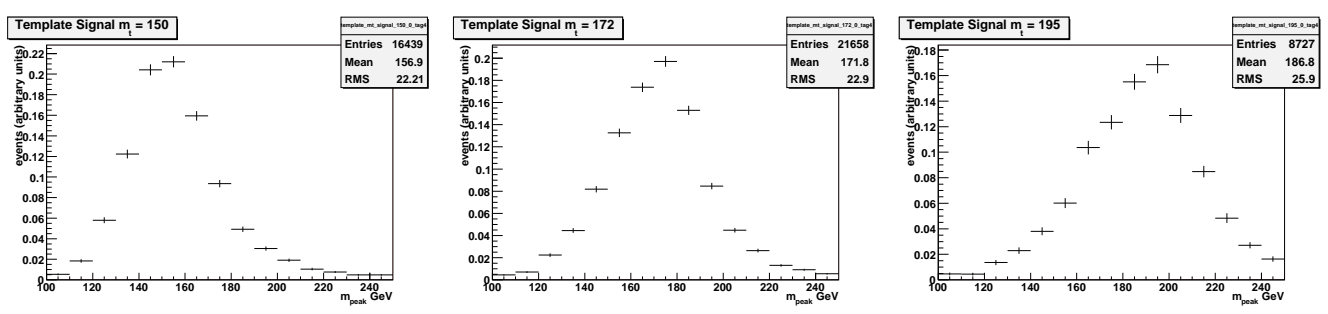

Figure 7.3: Templates from Monte Carlo events from $t \bar{t}$ decays to $e e$ for $m_{t}=150 \mathrm{GeV}$ (left), $172.5 \mathrm{GeV}$ (center), and $195 \mathrm{GeV}$ (right).
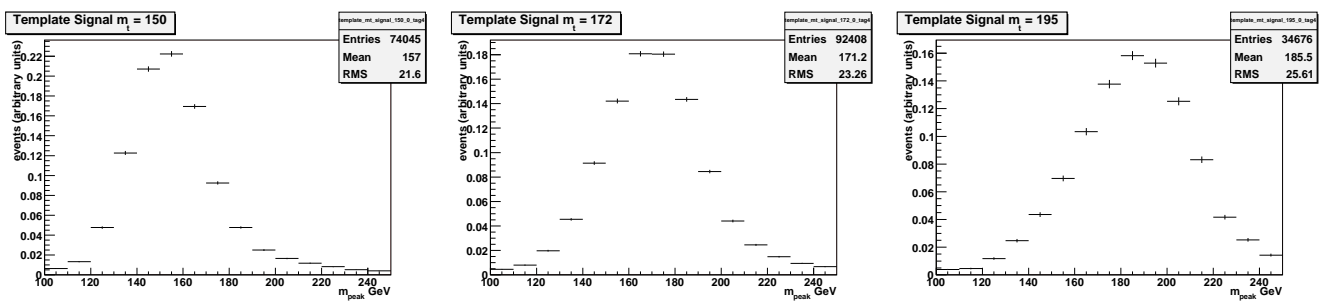

Figure 7.4: Templates from Monte Carlo events from $t \bar{t}$ decays to $e \mu$ for $m_{t}=150 \mathrm{GeV}$ (left), $172.5 \mathrm{GeV}$ (center), and $195 \mathrm{GeV}$ (right).
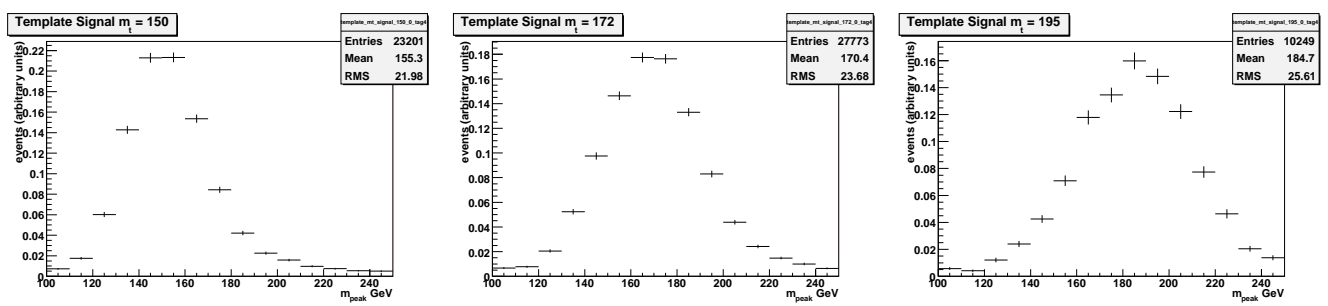

Figure 7.5: Templates from Monte Carlo events from $t \bar{t}$ decays to $\mu \mu$ for $m_{t}=150 \mathrm{GeV}$ (left), $172.5 \mathrm{GeV}$ (center), and $195 \mathrm{GeV}$ (right). 
data (see figures 7.1- 7.5 ). The distribution of measured top quark mass values from the ensemble fits gives an estimate of the parent distribution of our measurement.

We use multinomial statistics, the total number of events is fixed to the number observed in data for each channel, and each event is randomly drawn from the signal or Monte Carlo samples with a probability proportional to the number of events expected from each sample. Having chosen a sample to draw an event from, we choose a random event, and then accept or reject the event by comparing the event weight to a random number.

Figure 7.7 shows the results of the ensemble tests. Figure 7.6 shows the fitted mass, measured error and pull distribution for a top quark mass of $172.5 \mathrm{GeV}$. Where the pull is defined as the the measured value minus the true value, divided by the measured error. We have calibrated our results so as to remove an offset of $0.97 \mathrm{GeV}$ and slope of 0.99 , and the plots of mean errors and pull rms values reflect this calibration.
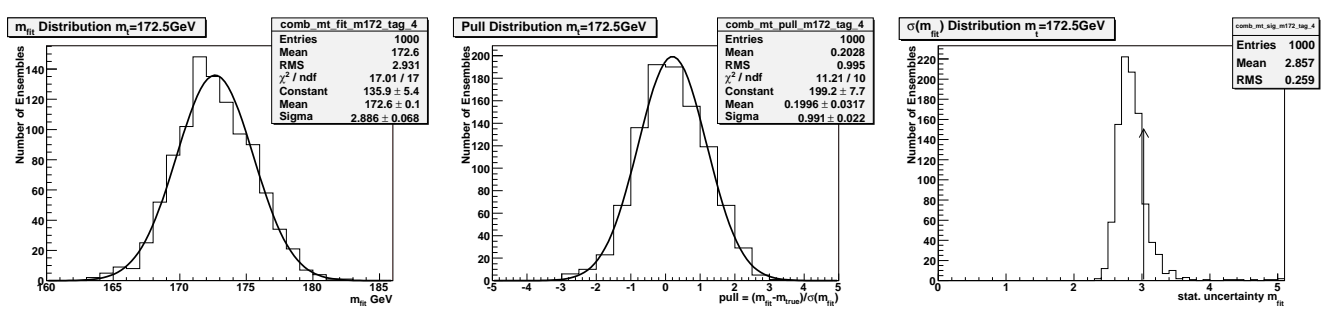

Figure 7.6: Fitted mass distribution, pull distribution, and error distribution for 1000 ensembles with $m_{t}=172.5 \mathrm{GeV}$ for combination of channels.
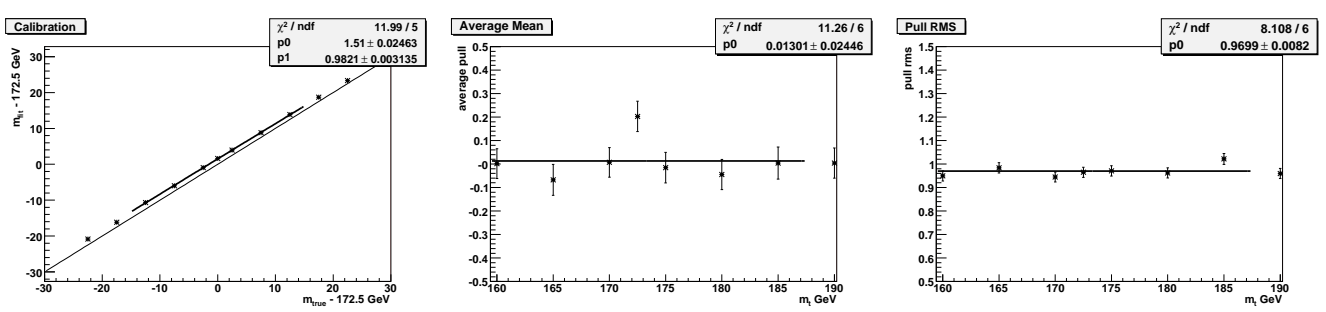

Figure 7.7: Average fit mass, pull, and pull width versus input top quark mass for the ensemble tests for combination of channels. 


\subsection{Result from DØ Data}

The fit results have to be corrected for the small offset observed in the calibration (see Figure 7.7) and for the pull widths.

The calibrated result for the combination of all channels in Run IIa, and Run IIb is:

$$
\begin{aligned}
& \text { Run IIa : } m_{t}=173.2 \pm 4.9(\text { stat }) \mathrm{GeV}, \\
& \text { Run IIb : } m_{t}=174.8 \pm 3.0(\text { stat }) \mathrm{GeV}
\end{aligned}
$$
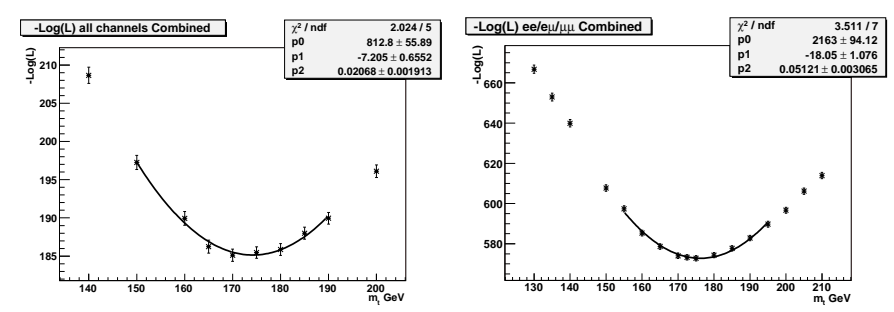

Figure 7.8: Plots of $-\ln L$ versus top quark mass for Run IIa (left) and Run IIb (right).
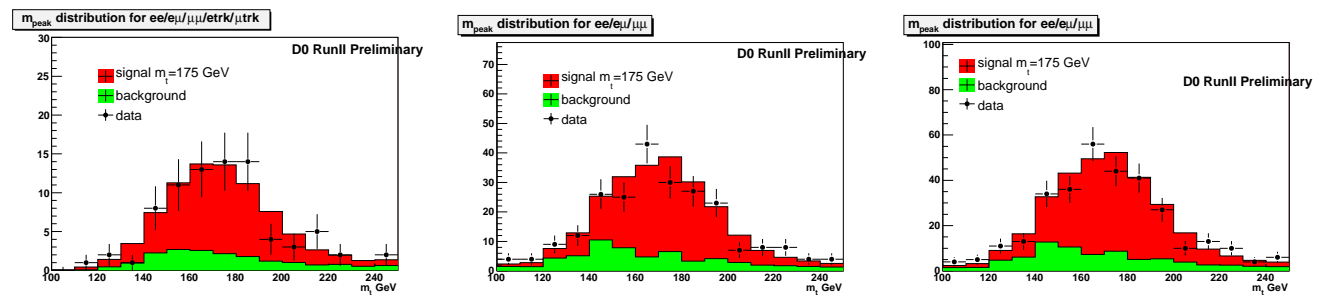

Figure 7.9: comparison of peak masses in data and Monte Carlo in Run IIa (left) Run IIb (center), and full Run II (right). 


\subsection{Mass Measurements Split into Channels}

\subsubsection{Run IIa Mass Measurement per Channel}

The calibrated results for the five channels separately are (see figure 7.28):

$$
\begin{aligned}
e e & : \quad m_{t}=180.1 \pm 10.2 \mathrm{GeV}, \\
e \mu & : \quad m_{t}=172.5 \pm 7.3 \mathrm{GeV}, \\
\mu \mu & : \quad m_{t}=199.5 \pm 19.8 \mathrm{GeV}, \\
\text { etrk } & : \quad m_{t}=159.0 \pm 11.9 \mathrm{GeV}, \\
\mu t r k & : \quad m_{t}=172.6 \pm 18.1 \mathrm{GeV} .
\end{aligned}
$$

The expected errors for the individual channels are:

$$
\begin{aligned}
e e & : 12.5 \mathrm{GeV}, \\
e \mu & : \quad 7.6 \mathrm{GeV}, \\
\mu \mu & : 22.2 \mathrm{GeV}, \\
\text { etrk } & : 17.1 \mathrm{GeV}, \\
\mu t r k & : 21.2 \mathrm{GeV} .
\end{aligned}
$$
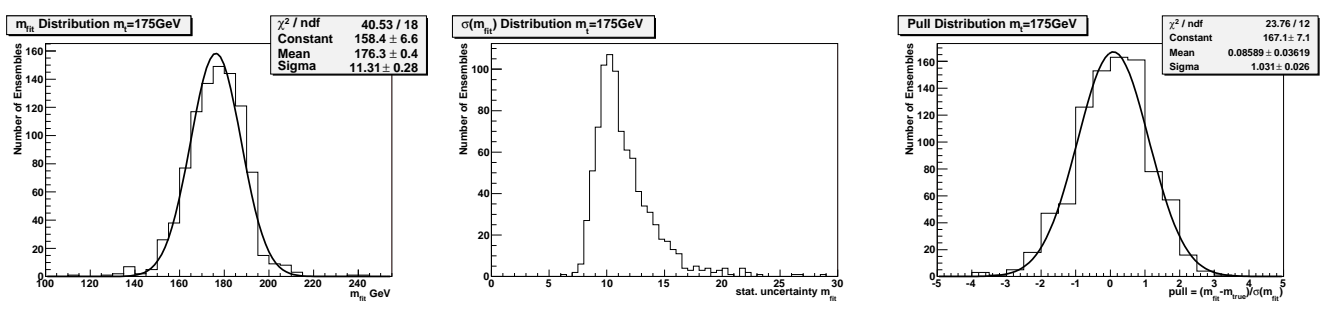

Figure 7.10: Fitted mass distribution, error distribution, and pull distribution for 1000 ensembles with $m_{t}=175 \mathrm{GeV}$ in ee channel. 

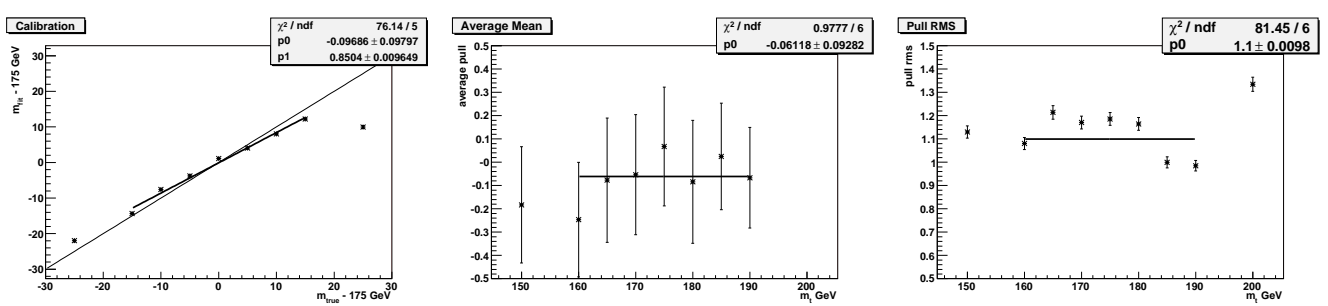

Figure 7.11: Average fit mass, pull, and pull width versus input top quark mass for the ensemble tests in ee channel.
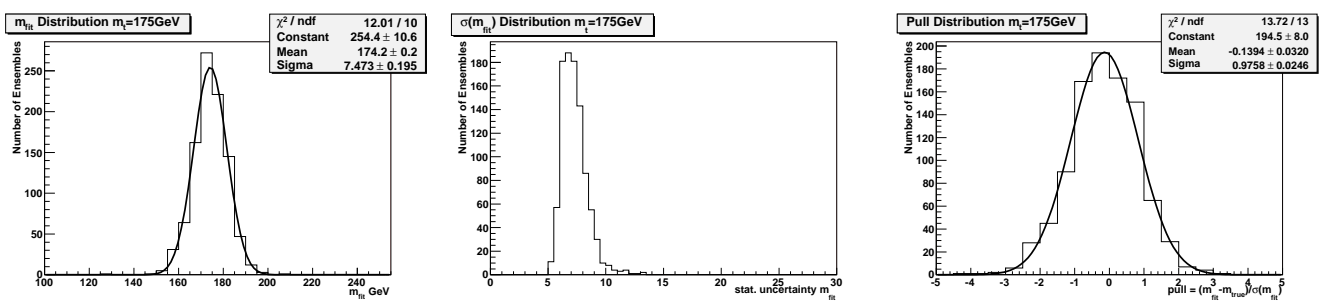

Figure 7.12: Fitted mass distribution, error distribution, and pull distribution for 1000 ensembles with $m_{t}=175 \mathrm{GeV}$ in $e \mu$ channel.
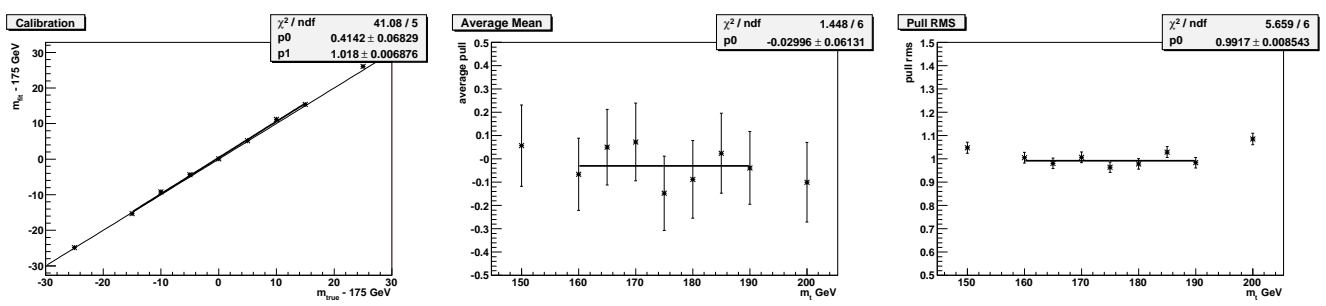

Figure 7.13: Average fit mass, pull, and pull width versus input top quark mass for the ensemble tests in $e \mu$ channel.
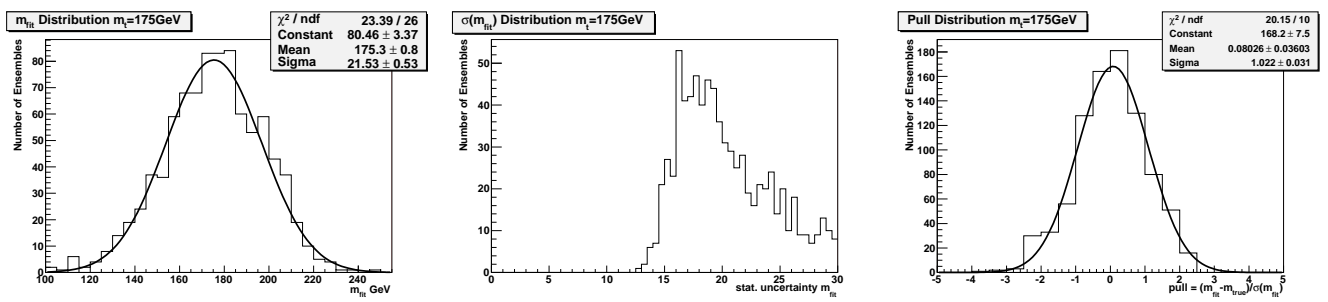

Figure 7.14: Fitted mass distribution, error distribution, and pull distribution for 1000 ensembles with $m_{t}=175 \mathrm{GeV}$ in $\mu \mu$ channel. 

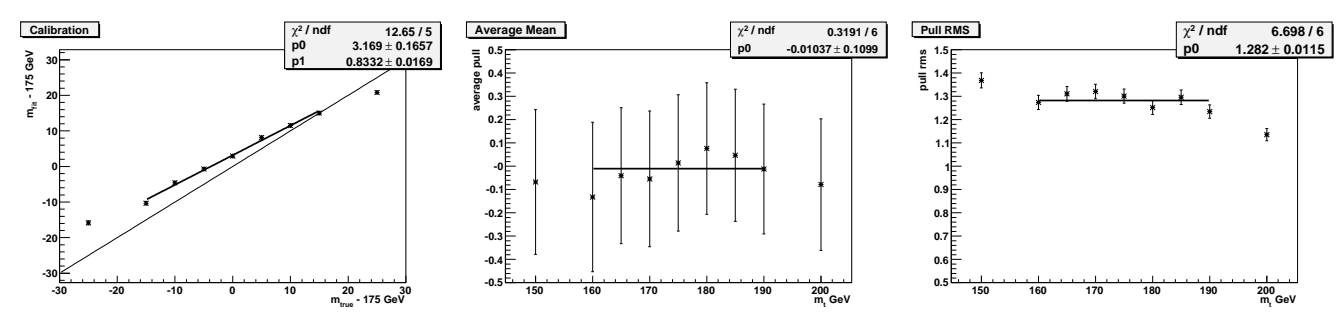

Figure 7.15: Average fit mass, pull, and pull width versus input top quark mass for the ensemble tests in $\mu \mu$ channel.
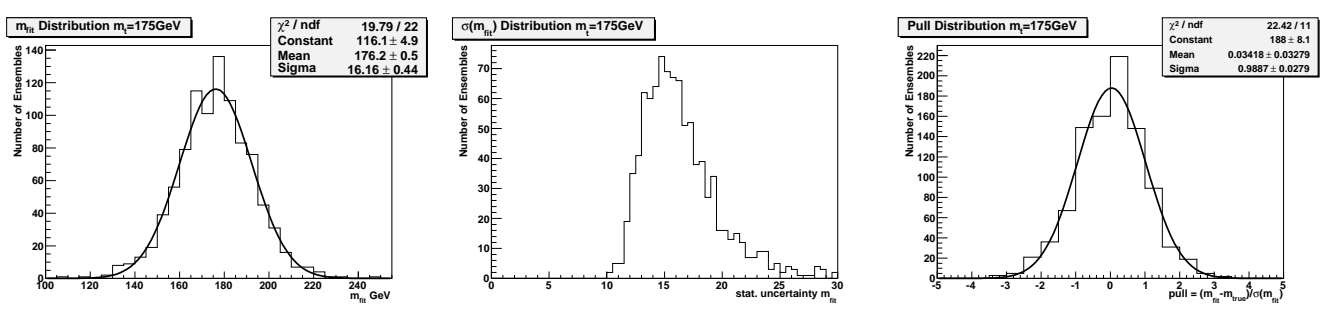

Figure 7.16: Fitted mass distribution, error distribution, and pull distribution for 1000 ensembles with $m_{t}=175 \mathrm{GeV}$ in $e+$ track channel.
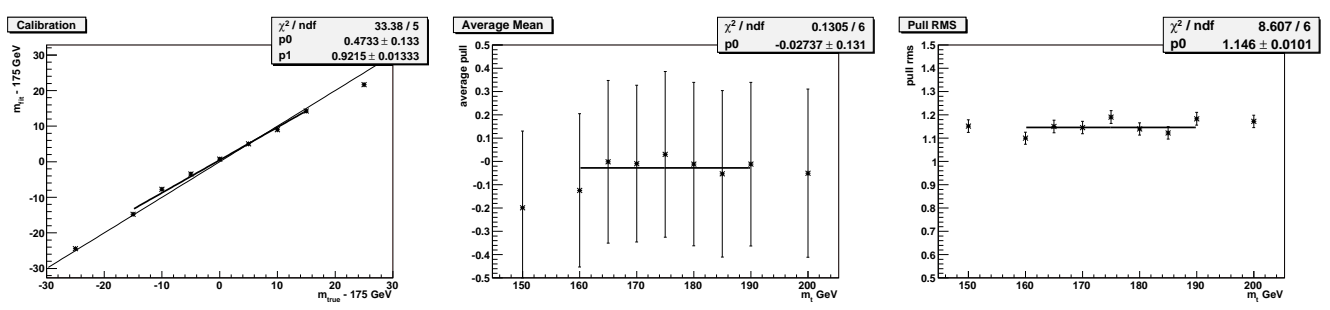

Figure 7.17: Average fit mass, pull, and pull width versus input top quark mass for the ensemble tests in $e+$ track channel.
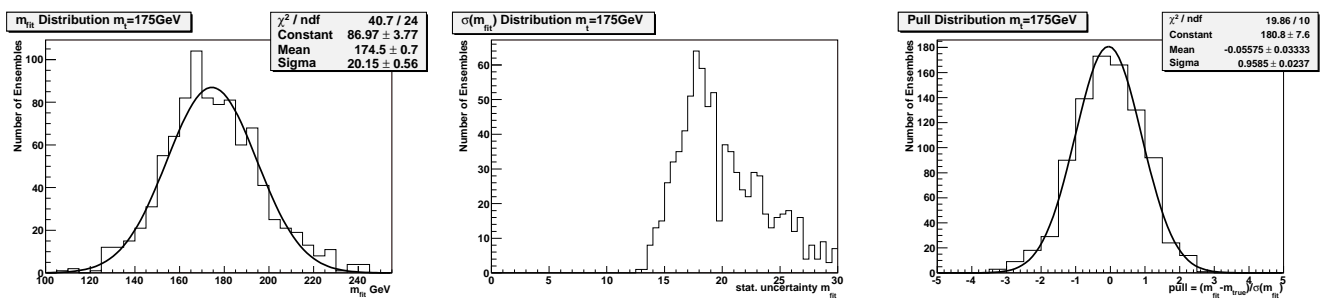

Figure 7.18: Fitted mass distribution, error distribution, and pull distribution for 1000 ensembles with $m_{t}=175 \mathrm{GeV}$ in $\mu+$ track channel. 

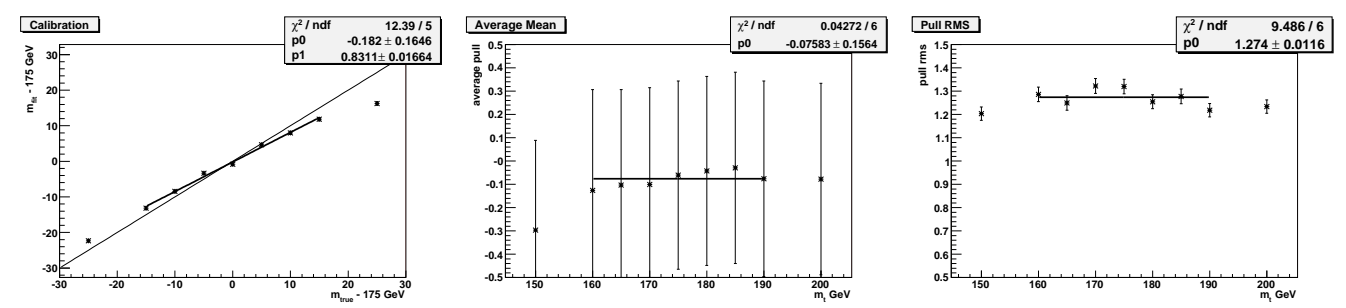

Figure 7.19: Average fit mass, pull, and pull width versus input top quark mass for the ensemble tests in $\mu+$ track channel.

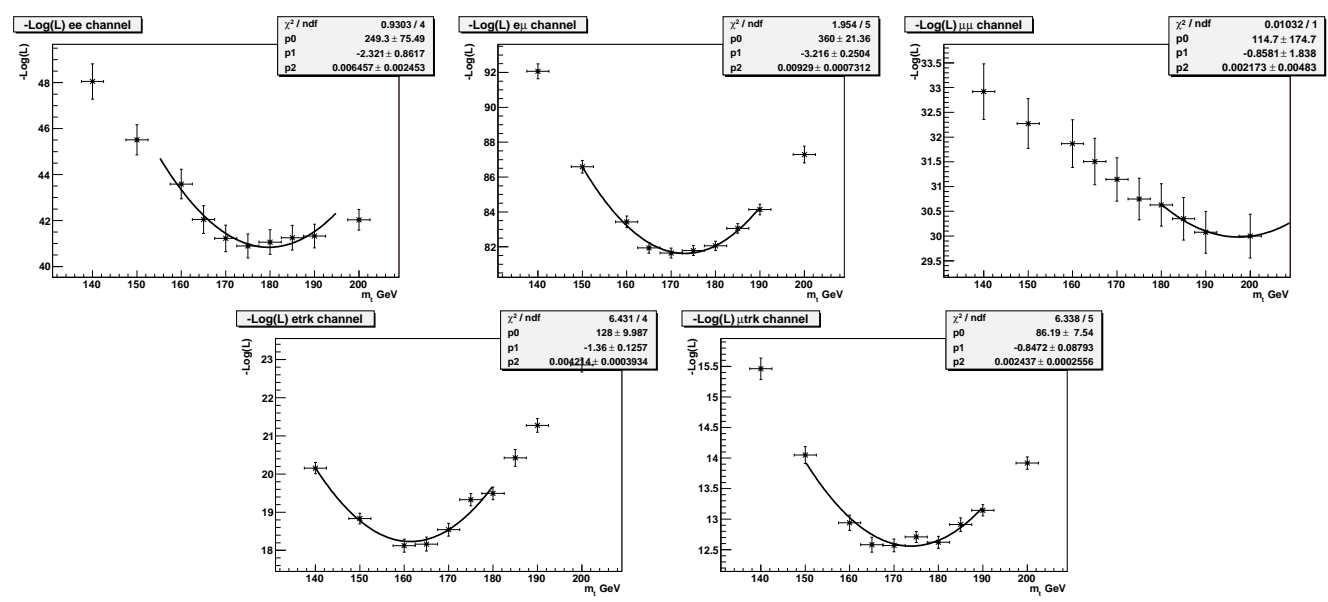

Figure 7.20: plots of $-\ln L$ versus top quark mass in the $e e, e \mu, \mu \mu, e+$ track and $\mu+$ track channels. 


\subsubsection{Run IIb Mass Measurement per Channel}

The calibrated results for the three channels separately are:

$$
\begin{aligned}
e e & : \quad m_{t}=184.2 \pm 8.1(\text { stat }) \pm 2.4(\text { syst }) \mathrm{GeV}, \\
e \mu & : \quad m_{t}=172.1 \pm 3.4(\text { stat }) \pm 2.0(\text { syst }) \mathrm{GeV}, \\
\mu \mu & : \quad m_{t}=182.3 \pm 13.2(\text { stat }) \pm 2.1(\text { syst }) \mathrm{GeV} .
\end{aligned}
$$

We have also provided separate systematics for the different channels (table 8.4), and a table of systematics for Run IIa and Run IIb (table 8.5).
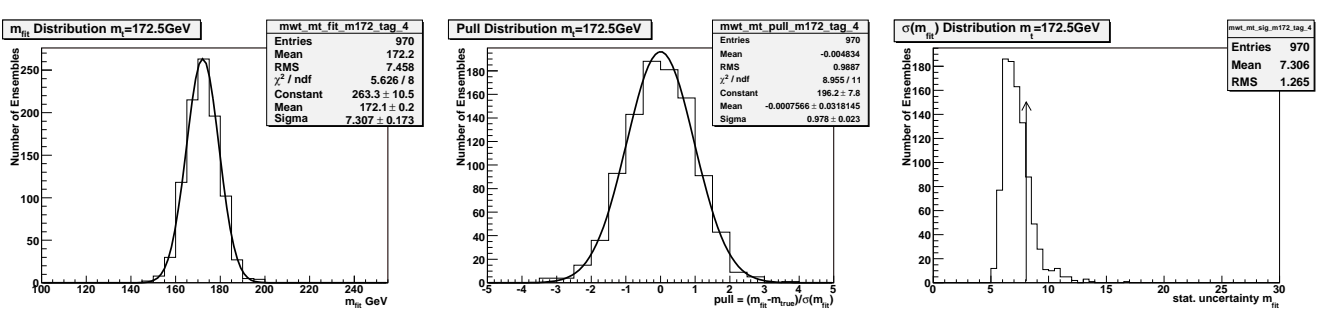

Figure 7.21: Fitted mass distribution, error distribution, and pull distribution for 1000 ensembles with $m_{t}=172.5 \mathrm{GeV}$ in ee channel.
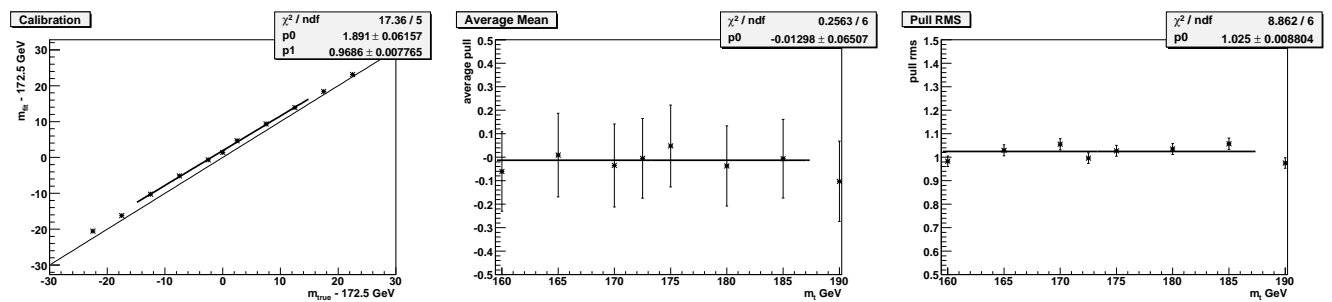

Figure 7.22: Average fit mass, pull, and pull width versus input top quark mass for the ensemble tests in ee channel. 

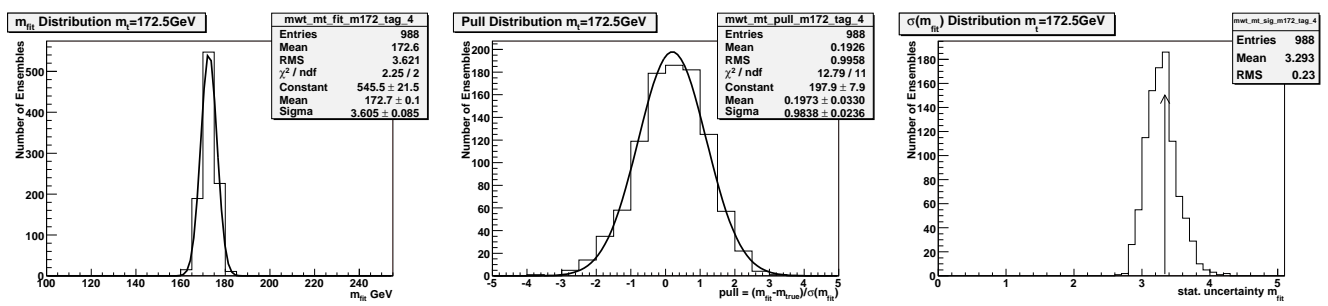

Figure 7.23: Fitted mass distribution, error distribution, and pull distribution for 1000 ensembles with $m_{t}=172.5 \mathrm{GeV}$ in $e \mu$ channel.
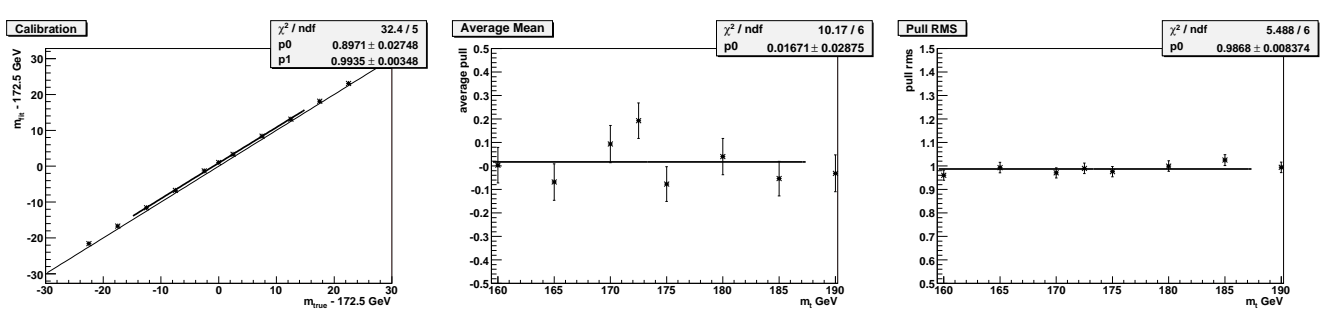

Figure 7.24: Average fit mass, pull, and pull width versus input top quark mass for the ensemble tests in $e \mu$ channel.
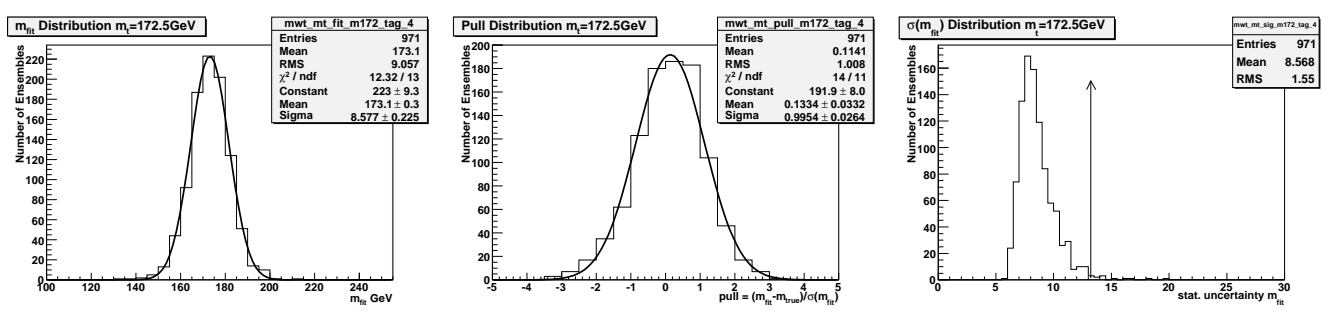

Figure 7.25: Fitted mass distribution, error distribution, and pull distribution for 1000 ensembles with $m_{t}=172.5 \mathrm{GeV}$ in $\mu \mu$ channel.
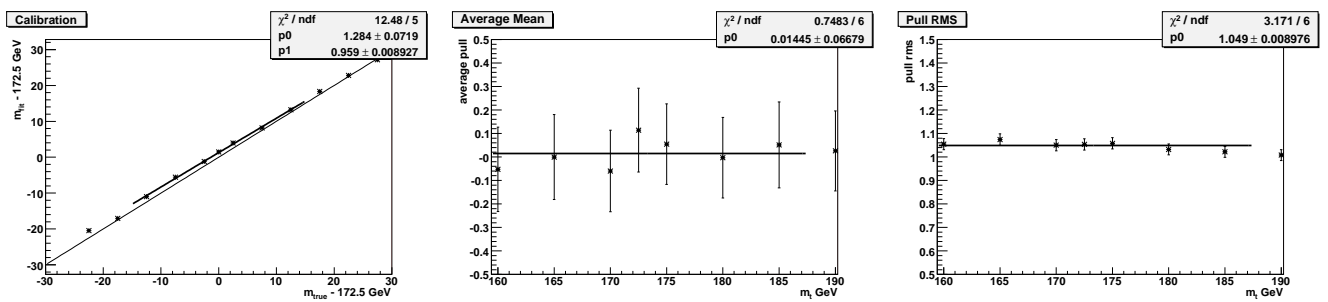

Figure 7.26: Average fit mass, pull, and pull width versus input top quark mass for the ensemble tests in $\mu \mu$ channel. 

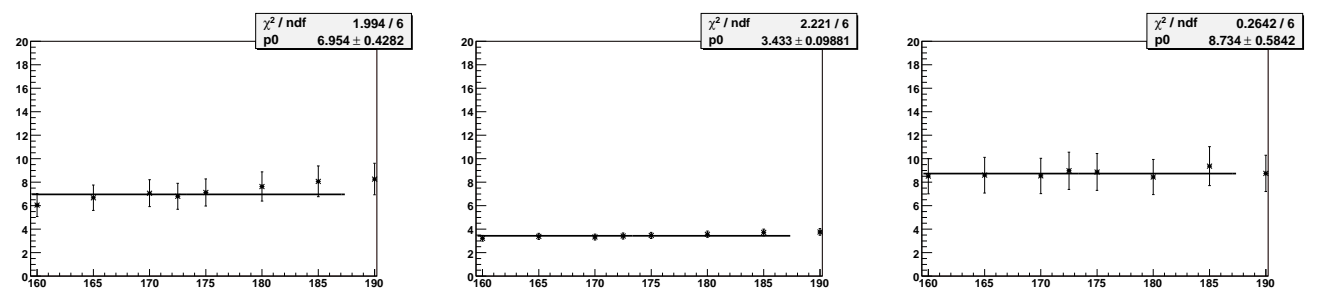

Figure 7.27: Average RMS of fitted mass distributions for $e e, e \mu$, and $\mu \mu$ channels.
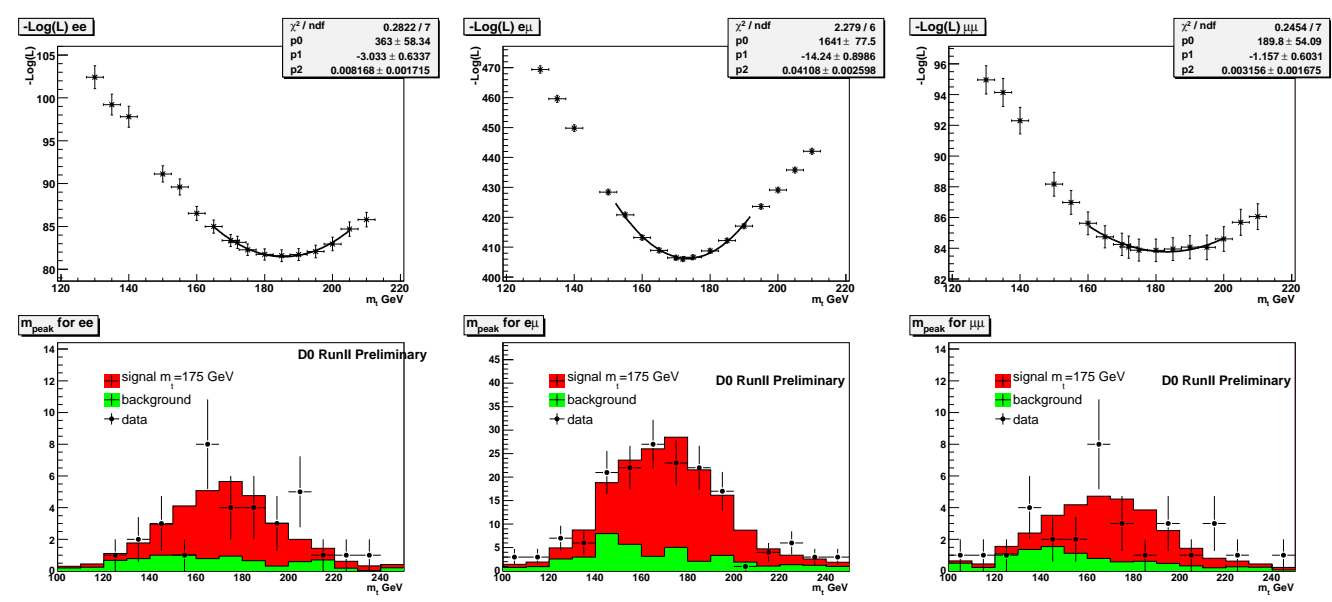

Figure 7.28: Plots of $-\ln L$ versus top quark mass and comparison of peak masses in data and Monte Carlo in the $e e, e \mu$, and $\mu \mu$ channels.
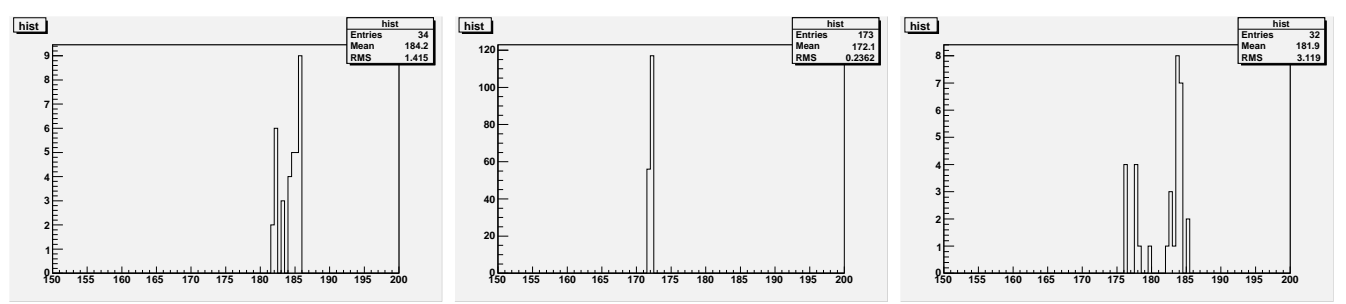

Figure 7.29: Variation in measured top mass in data due to removal of one event in $e e, e \mu$, and $\mu \mu$ channels. 


\section{Chapter 8}

\section{Systematic Uncertainties}

We use the ensemble test technique to study the size of the systematic uncertainties. We make systematic changes to the events in the ensembles, these changes can take the form of: changes to the kinematics of the event (e.g. changing $p_{T}$ of jets), changes to the samples used for the signal or background, changes to the event weights, which then change the composition of the pseudo-experiments. We then fit them using the nominal templates. The change in the result gives the size of the systematic uncertainty. A description of the systematics follows, and a summary can be found in table 8.2.

\subsection{Jet Reconstruction}

\subsubsection{Jet Energy Scale}

Since we compare the results from the collider data against simulated templates, the measurement will be systematically biased if the jet energies are calibrated differently in data and simulation. Before applying the standard Jet Smearing and Removal procedure [32], we vary all jet energies by $\pm 1 \sigma$ using errors parametrized in terms of the jet $p_{T}$ and $\eta$. We then apply the selection cuts and perform ensemble tests using the two varied samples. We compare the results with the nominal sample and find the variation in $m_{t}$ from this change to be $\pm 1.5 \mathrm{GeV}$. 


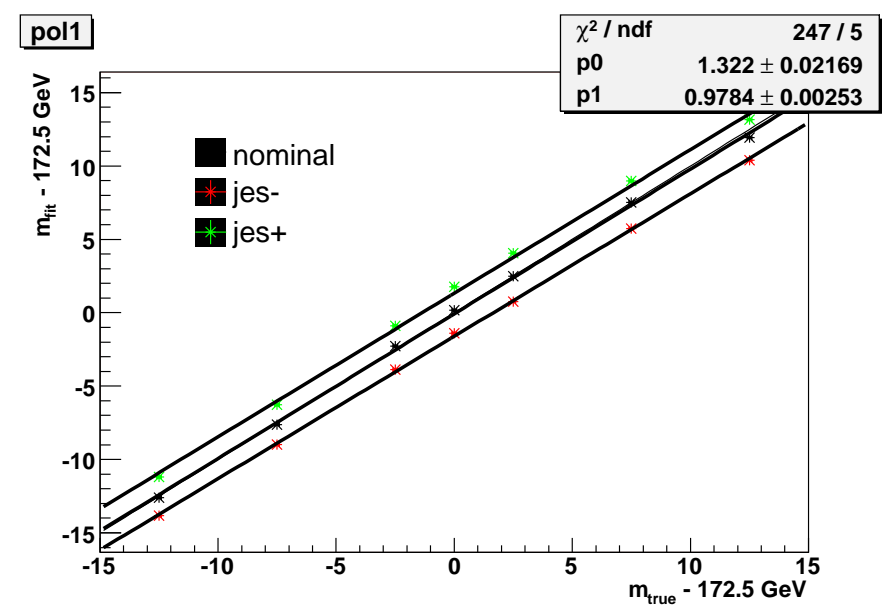

Figure 8.1: Average fit mass versus input top quark mass for ensemble tests with the jet scale varied by $\pm 1 \sigma$.

\subsection{2 b/l Response and Sample-Dependent Response}

The jet energy scale is derived from $\gamma+$ jet events. As outlined in section 3.3.5, differences between the single pion response in data and Monte Carlo lead to a systematic shift in the jet energy for $b, c$, and light jets in $t \bar{t}$. The shift is parameterized by $k_{b}, k_{c}$, and $k_{l}$, where these multiplicative factors are applied to the energy of each jet. This shift is broken up into two components, the shift from a mixture of quark and gluon jets to mostly quark jets, which we call the sample dependent correction, where each jet's energy is multiplied by $k_{l}$, a shift of $-0.4 \mathrm{GeV}$ in the measured $m_{t}$, and the other the shift from light quark jets to b-jets, where each jet's energy is multiplied by $k_{b} / k_{l}$, a shift of $+1.1 \mathrm{GeV}$ in the measured $m_{t}$. The difference in $m_{t}$ between the corrected and uncorrected samples is taken as the systematic uncertainty. It should be noted that these two systematics are correlated, and adding them together yields a shift of $+0.7 \mathrm{GeV}$ in $m_{t}$.

\subsection{3 b-Fragmentation}

B-Fragmentation: This systematic is estimated by reweighting events according to two different b-fragmentation parametrization (Bowler AOD [59] is used for all events, Bowler SLD [59] is used as systematic), a shift of $\pm 0.01 \mathrm{GeV}$ is found. 


\subsection{Signal Modeling}

There are various uncertainties in the modeling of soft-QCD effects. These include hadronization modeling, underlying event modeling, modeling of the primordial transverse momentum of the incoming partons, modeling of QCD color correlations, modeling of color reconnection.

\subsubsection{Hard Scatter}

We estimate this effect by comparing a sample for which the hard-scatter is generated using ALPGEN and PYTHIA is used for hadronization to a sample for which PYTHIA is used for both hard-scatter and hadronization. We estimate the size of this first component to be $\pm 0.2 \mathrm{GeV}$.

\subsubsection{Color Reconnection}

We estimate the effect of the modeling of color reconnection by comparing our default PYTHIA tune (tune A) to tune ACR [60] which is tune A with modified color reconnection model. This gives a shift of $\pm 0.1 \mathrm{GeV}$.

\subsubsection{Particle Showering Tune}

We estimate the effect of uncertainties in the underlying event modeling, the modeling of the primordial transverse momentum of the incoming partons, and the modeling of QCD color correlations by comparing tune A to tune S0 [60]. this gives a shift of $\pm 0.3 \mathrm{GeV}$.

\subsubsection{Higher Order Effects}

We use a leading order (LO) event generator in our analysis. We estimate the effect of not including higher order effects by comparing with the next to leading order (NLO) generator MC@NLO. The shift in $m_{t}$ between the two samples is $\pm 0.3 \mathrm{GeV}$. 


\subsubsection{Hadronization}

We estimate the effect of uncertainties in the hadronization model by comparing the PYTHIA event generator with the HERWIG event generator. We find a shift of $\pm 0.6 \mathrm{GeV}$.

\subsubsection{Initial and Final State Radiation}

We first use a data based method. We modify the ratio of events with three or more jets to events with two jets. A variation of $20 \%$ is chosen based on the difference between the ratio observed in data and the number expected from the Monte Carlo. Events with three jets are then reweighted to increase their contribution to the sample. The variation in measured top quark mass obtained in this way is $\pm 0.1 \mathrm{GeV}$. Next we use a Monte Carlo based method, we use samples generated with PYTHIA and the CTEQ5L PDF set [61], then we compare this to samples with CTEQ5L for which ISR/FSR have been increased and decreased. We find a variation for the Monte Carlo based method of $\pm 0.1 \mathrm{GeV}$.

\subsubsection{Multiple Interactions}

The instantaneous luminosity distributions in Data and Monte Carlo are known to be substantially different, and we reweight the luminosity in Monte Carlo to match the luminosity profile in data. We assign a systematic in two ways, the first is to vary the reweighting histogram within its statistical errors, the second is to allow the reweighting procedure to apply an arbitrarily large weight (rather than restricting the weight to $\leq 3$, see section 5.3.3). This variation is found to be $\pm 0.01 \mathrm{GeV}$.

\subsubsection{Parton Distribution Function}

We estimate the effect of the uncertainty in the parton distribution functions by reweighting the Monte Carlo events in our ensembles up and down according to the 20 available error sets in the CTEQ6.1 PDF [62] and then adding the systematic uncertainties obtained from each in quadrature. The uncertainty obtained in this way is $\pm 0.4 \mathrm{GeV}$. This result was obtained using PYTHIA signal Monte Carlo, and is consistent with the result obtained 
using ALPGEN Monte Carlo.

\subsection{Detector Model}

\subsubsection{Jet Energy Resolution}

The smearing applied to Monte Carlo jets is varied up and down within its uncertainties. This is found to shift the top mass by $\pm 0.03 \mathrm{GeV}$.

\subsubsection{Jet Identification Efficiency}

Calorimeter clusters which fail the standard jet requirements are removed in data and Monte Carlo. The efficiency for jet identification is slightly higher in Monte Carlo, and

jets are randomly removed in Monte Carlo to account for this difference. The number of jets removed is changed to account the known systematic error in the $p_{T}$ and $\eta$ dependent scale factor used in removing Monte Carlo jets. This change in jet removal introduces a systematic shift of $+0.01 \mathrm{GeV}$.

\subsubsection{Electron, Muon Identification}

The efficiencies for an electron or muon to pass the electron or muon[47] identification cuts differ in data and Monte Carlo. We apply a scale factor to account for this difference, and vary these scale factors up and down by $1 \sigma$ to obtain a systematic. The shift for electrons is $\pm 0.02 \mathrm{GeV}$ and the shift for muons is $\pm 0.02 \mathrm{GeV}$.

\subsubsection{Z-position Profile of Primary Event Vertex}

The $z$ vertex distribution in data and Monte Carlo are known to be different[44]. We reweight the $z$ vertex distribution in Monte Carlo to match that in data. The nominal reweighting is done using parameters fit within the range $|z|<60 \mathrm{~cm}$. We use parameters derived over the range $z<40 \mathrm{~cm}$ to estimate a systematic, for a shift of $+0.01 \mathrm{GeV}$. 


\subsection{Lepton Momentum Scale and Resolution}

\subsubsection{Muon Momentum Resolution}

The resolution of the muon momentum measurement in data and Monte Carlo is known to be different. To account for this difference we smear the $p_{T}$ of muons in our Monte Carlo[23][24]. The parameters are determined through a fitting procedure. We shift the smearing parameters up and down by their know systematic errors, and obtain a systematic shift of $\pm 0.02 \mathrm{GeV}$.

\subsubsection{Electron Energy Resolution}

The resolution of the electron momentum measurement in data and Monte Carlo is known to be different. To account for this difference we smear the $p_{T}$ of muons in our Monte Carlo[1]. The parameters are determined through a fitting procedure, and we shift the smearing parameters up and down by their known systematic errors, and obtain a systematic shift of $\pm 0.1 \mathrm{GeV}$.

\subsubsection{Muon Momentum Scale}

We scale the momentum of the muon according to distributions in [24], according to either a linear or quadratic fit. We find a systematic shift of $\pm 0.1 \mathrm{GeV}$.

\subsubsection{Electron Momentum Scale}

We scale the energy of the electron according to the function:

$$
0.0751073-0.0267345 \log (E)+0.00190248 \log ^{2}(E) .
$$

We find a systematic shift of $\pm 0.2 \mathrm{GeV}$ in the top mass. 


\subsection{Background Model}

\subsubsection{Background Normalization}

We vary the number of background events in our ensembles up and down by the statistical error from tables 6.2, 6.4, and 6.6. We also vary the number of signal events according to the error from the measured cross section. These errors are added together in quadrature, and we find a total systematic shift of $\pm 0.2 \mathrm{GeV}$.

\subsubsection{Physics Background Model}

To estimate this systematic, we replace all of the ALPGEN+PYTHIA generated $Z+j e t$ samples in our background with equivalent PYTHIA samples. The systematic variation of the top mass is found to be $\pm 0.2 \mathrm{GeV}$.

\subsubsection{Fake Background Model}

We estimate the contribution of events with fake leptons to the background by parameterizing the real and fake efficiencies as a function of $p_{T}$ (see figures 6.1-6.4 and 8.2). In forming the normalized background templates (see section 7.4), we use the fake rate estimate described in section 6.1.5 separately for each bin to determine the shape of the fake background. This bin-by-bin method is compared to the shape obtained from a sample of loose same-sign events (Fig. 8.3). We find the shift to be a negligible $0.01 \mathrm{GeV}$. We've also look at the variation of the efficiencies with the jet multiplicity (Table 8.1). We find a total shift of $0.1 \mathrm{GeV}$.

\begin{tabular}{l|c|c|c}
\hline & $e e$ & $e \mu$ & $\mu \mu$ \\
\hline inclusive & $1.33 \pm 0.10$ & $13.98 \pm 0.65$ & $2.10 \pm 0.14$ \\
$\geq 2$ & $1.51 \pm 0.50$ & $9.59 \pm 1.82$ & $1.62 \pm 0.22$ \\
\hline
\end{tabular}

Table 8.1: Number of fake events for efficiencies obtained with inclusive jets, and $\geq 2$ jets, see Fig. 6.1-6.4 for dependence of efficiencies on jet multiplicity. 

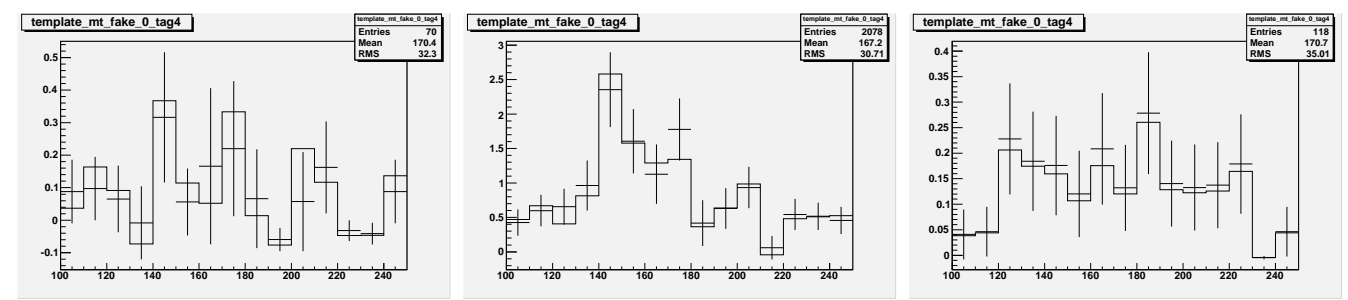

Figure 8.2: Nominal fake templates compared with templates using $p_{T}$ dependent fake rate $(e e, e \mu, \mu \mu)$.
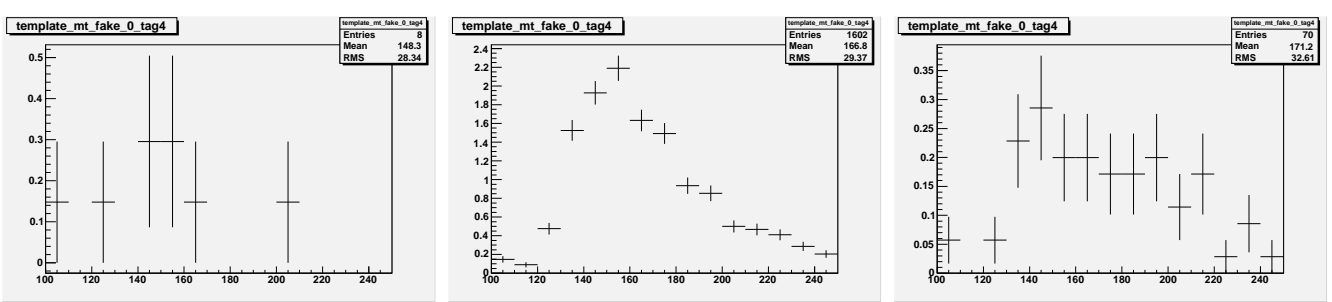

Figure 8.3: Fake templates obtained from loose same-sign samples $(e e, e \mu, \mu \mu)$.

\subsection{Method}

\subsubsection{Template Statistics}

Two methods were considered. In the first method the events used to form templates are split into four subsamples, ensemble tests are performed separately using each part, and the rms of the variations is found to be $\pm 0.1 \mathrm{GeV}$. In the second method the $-\log \mathcal{L}$ values are varied up and down within their uncertainties, using this method we also obtain a systematic error of $\pm 0.1 \mathrm{GeV}$.

\subsubsection{Ensemble Statistics}

We use a finite number of ensembles, and draw from a finite number of Monte Carlo events. We estimate the statistical uncertainty in the calibration of our measurement originating from the finite sample sizes by fitting the difference between the fitted mass and input mass to a constant value and taking the error on the fit as the systematic. The variation is found to be $\pm 0.04 \mathrm{GeV}$. 
Table 8.2: Summary of uncertainties.

\begin{tabular}{lcr} 
source & Run IIa & Run IIb \\
\hline jet energy scale & $1.2 \mathrm{GeV}$ & $1.5 \mathrm{GeV}$ \\
b/l response + sample dep & $0.4 \mathrm{GeV}$ & $0.4 \mathrm{GeV}$ \\
b-fragmentation systematic & $0.01 \mathrm{GeV}$ & $0.01 \mathrm{GeV}$ \\
\hline signal model: hard scatter & $0.2 \mathrm{GeV}$ & $0.2 \mathrm{GeV}$ \\
signal model: color reconnection & $0.1 \mathrm{GeV}$ & $0.1 \mathrm{GeV}$ \\
signal model: particle showering tune & $0.3 \mathrm{GeV}$ & $0.3 \mathrm{GeV}$ \\
higher order effects (MC@NLO) & $0.3 \mathrm{GeV}$ & $0.3 \mathrm{GeV}$ \\
hadronization & $0.6 \mathrm{GeV}$ & $0.6 \mathrm{GeV}$ \\
ISR/FSR & $0.3 \mathrm{GeV}$ & $0.17 \mathrm{GeV}$ \\
multiple interactions & $0.2 \mathrm{GeV}$ & $0.01 \mathrm{GeV}$ \\
pdf & $0.5 \mathrm{GeV}$ & $0.4 \mathrm{GeV}$ \\
\hline jet energy resolution & $0.2 \mathrm{GeV}$ & $0.03 \mathrm{GeV}$ \\
jetid & $0.5 \mathrm{GeV}$ & $0.01 \mathrm{GeV}$ \\
emid & $0.1 \mathrm{GeV}$ & $0.02 \mathrm{GeV}$ \\
$\mu$ id & $0.1 \mathrm{GeV}$ & $0.02 \mathrm{GeV}$ \\
beam $|z|$ reweighting & $0.0 \mathrm{GeV}$ & $0.01 \mathrm{GeV}$ \\
\hline$\mu$ resolution & $0.2 \mathrm{GeV}$ & $0.02 \mathrm{GeV}$ \\
em resolution & $0.2 \mathrm{GeV}$ & $0.1 \mathrm{GeV}$ \\
$\mu$ scale & $0.1 \mathrm{GeV}$ & $0.1 \mathrm{GeV}$ \\
em scale & $0.2 \mathrm{GeV}$ & $0.2 \mathrm{GeV}$ \\
\hline background yield & $0.1 \mathrm{GeV}$ & $0.2 \mathrm{GeV}$ \\
background model & $0.3 \mathrm{GeV}$ & $0.2 \mathrm{GeV}$ \\
fake background shape & $0.1 \mathrm{GeV}$ & $0.1 \mathrm{GeV}$ \\
template statistics & $0.8 \mathrm{GeV}$ & $0.1 \mathrm{GeV}$ \\
ensemble statistics & $0.1 \mathrm{GeV}$ & $0.04 \mathrm{GeV}$ \\
\hline total systematic error & $2.0 \mathrm{GeV}$ & $1.8 \mathrm{GeV}$ \\
\hline expected statistical error & $5.8 \mathrm{GeV}$ & $3.0 \mathrm{GeV}$ \\
observed statistical error & $4.9 \mathrm{GeV}$ & $3.0 \mathrm{GeV}$ \\
\hline total expected error & $6.2 \mathrm{GeV}$ & $3.5 \mathrm{GeV}$ \\
total observed & $5.3 \mathrm{GeV}$ & $3.6 \mathrm{GeV}$ \\
& & \\
\hline
\end{tabular}




\subsection{Systematic Uncertainties per Channel}

Table 8.3: Summary of uncertainties for Run IIa, combined, dilepton, lepton+track channels separately.

\begin{tabular}{lccr} 
Source & combined & dilepton & lepton+track \\
\hline jet energy scale & $1.2 \mathrm{GeV}$ & $1.2 \mathrm{GeV}$ & $1.2 \mathrm{GeV}$ \\
bJES/sample dependent JES & $0.6 \mathrm{GeV}$ & $0.6 \mathrm{GeV}$ & $0.6 \mathrm{GeV}$ \\
jet energy resolution & $0.2 \mathrm{GeV}$ & $0.2 \mathrm{GeV}$ & $0.2 \mathrm{GeV}$ \\
\hline signal modeling - ALPGEN & $0.5 \mathrm{GeV}$ & $0.5 \mathrm{GeV}$ & $0.5 \mathrm{GeV}$ \\
signal modeling - PYTHIA tune & $0.5 \mathrm{GeV}$ & $0.5 \mathrm{GeV}$ & $0.5 \mathrm{GeV}$ \\
b-tagging systematic & $0.2 \mathrm{GeV}$ & $0.2 \mathrm{GeV}$ & $0.3 \mathrm{GeV}$ \\
b-fragmentation systematic & $0.1 \mathrm{GeV}$ & $0.2 \mathrm{GeV}$ & $0.4 \mathrm{GeV}$ \\
gluon radiation & $0.1 \mathrm{GeV}$ & $0.2 \mathrm{GeV}$ & $0.3 \mathrm{GeV}$ \\
template statistics & $0.5 \mathrm{GeV}$ & $0.6 \mathrm{GeV}$ & $0.5 \mathrm{GeV}$ \\
pdf & $0.5 \mathrm{GeV}$ & $0.5 \mathrm{GeV}$ & $0.8 \mathrm{GeV}$ \\
instantaneous luminosity & $0.2 \mathrm{GeV}$ & $0.2 \mathrm{GeV}$ & $0.2 \mathrm{GeV}$ \\
ensemble statistics & $0.1 \mathrm{GeV}$ & $0.1 \mathrm{GeV}$ & $0.2 \mathrm{GeV}$ \\
\hline background normalization & $0.1 \mathrm{GeV}$ & $0.1 \mathrm{GeV}$ & $0.2 \mathrm{GeV}$ \\
background model & $0.3 \mathrm{GeV}$ & $0.3 \mathrm{GeV}$ & $0.3 \mathrm{GeV}$ \\
em resolution & $0.1 \mathrm{GeV}$ & $0.1 \mathrm{GeV}$ & $0.2 \mathrm{GeV}$ \\
$\mu$ resolution & $0.2 \mathrm{GeV}$ & $0.2 \mathrm{GeV}$ & $0.2 \mathrm{GeV}$ \\
\hline \hline total systematic error & $1.8 \mathrm{GeV}$ & $1.8 \mathrm{GeV}$ & $2.0 \mathrm{GeV}$ \\
\hline expected statistical uncertainty & $5.8 \mathrm{GeV}$ & $6.3 \mathrm{GeV}$ & $12.7 \mathrm{GeV}$ \\
observed statistical error & $4.9 \mathrm{GeV}$ & $5.5 \mathrm{GeV}$ & $8.5 \mathrm{GeV}$ \\
\hline total expected error & $6.1 \mathrm{GeV}$ & $6.6 \mathrm{GeV}$ & $12.9 \mathrm{GeV}$ \\
total observed & $5.2 \mathrm{GeV}$ & $5.8 \mathrm{GeV}$ & $8.7 \mathrm{GeV}$
\end{tabular}


Table 8.4: Summary of uncertainties for Run IIb, $e e, e \mu, \mu \mu$, combined.

\begin{tabular}{lrrrr} 
Source & $e e$ & $e \mu$ & $\mu \mu$ & $c o m b$ \\
\hline jet energy scale & $1.5 \mathrm{GeV}$ & $1.6 \mathrm{GeV}$ & $1.7 \mathrm{GeV}$ & $1.5 \mathrm{GeV}$ \\
b/1 ratio & $0.6 \mathrm{GeV}$ & $0.4 \mathrm{GeV}$ & $0.6 \mathrm{GeV}$ & $0.4 \mathrm{GeV}$ \\
b-fragmentation & $0.1 \mathrm{GeV}$ & $0.02 \mathrm{GeV}$ & $0.1 \mathrm{GeV}$ & $0.01 \mathrm{GeV}$ \\
& & & & \\
& & & & \\
& & & \\
color reconnection & $0.2 \mathrm{GeV}$ & $0.2 \mathrm{GeV}$ & $0.2 \mathrm{GeV}$ & $0.1 \mathrm{GeV}$ \\
particle showering tune & $0.2 \mathrm{GeV}$ & $0.4 \mathrm{GeV}$ & $0.01 \mathrm{GeV}$ & $0.3 \mathrm{GeV}$ \\
higher order effects & $0.2 \mathrm{GeV}$ & $0.3 \mathrm{GeV}$ & $0.4 \mathrm{GeV}$ & $0.3 \mathrm{GeV}$ \\
(MC @LO) & & & & \\
hadronization & $0.6 \mathrm{GeV}$ & $0.6 \mathrm{GeV}$ & $0.6 \mathrm{GeV}$ & $0.6 \mathrm{GeV}$ \\
ISR/FSR from data & $0.3 \mathrm{GeV}$ & $0.1 \mathrm{GeV}$ & $0.03 \mathrm{GeV}$ & $0.1 \mathrm{GeV}$ \\
ISR/FSR CTEQ51 & $0.4 \mathrm{GeV}$ & $0.2 \mathrm{GeV}$ & $0.5 \mathrm{GeV}$ & $0.1 \mathrm{GeV}$ \\
multiple interactions & $0.04 \mathrm{GeV}$ & $0.06 \mathrm{GeV}$ & $0.2 \mathrm{GeV}$ & $0.01 \mathrm{GeV}$ \\
pdf & $0.4 \mathrm{GeV}$ & $0.4 \mathrm{GeV}$ & $0.5 \mathrm{GeV}$ & $0.4 \mathrm{GeV}$
\end{tabular}

\begin{tabular}{|c|c|c|c|c|}
\hline jet energy resolution & $0.2 \mathrm{GeV}$ & $0.1 \mathrm{GeV}$ & $0.1 \mathrm{GeV}$ & $0.03 \mathrm{GeV}$ \\
\hline jetid & $-0.02 \mathrm{GeV}$ & $-0.01 \mathrm{GeV}$ & $-0.02 \mathrm{GeV}$ & $+0.01 \mathrm{GeV}$ \\
\hline emid & $0.1 \mathrm{GeV}$ & $0.04 \mathrm{GeV}$ & & $0.02 \mathrm{GeV}$ \\
\hline$\mu \mathrm{id}$ & & $0.2 \mathrm{GeV}$ & $0.2 \mathrm{GeV}$ & $0.02 \mathrm{GeV}$ \\
\hline beam $|z|$ reweighting & $0.1 \mathrm{GeV}$ & $0.1 \mathrm{GeV}$ & $0.1 \mathrm{GeV}$ & $0.01 \mathrm{GeV}$ \\
\hline em resolution & $0.2 \mathrm{GeV}$ & $0.1 \mathrm{GeV}$ & & $0.1 \mathrm{GeV}$ \\
\hline$\mu$ scale & & $0.1 \mathrm{GeV}$ & $0.2 \mathrm{GeV}$ & $0.1 \mathrm{GeV}$ \\
\hline background yield & $0.2 \mathrm{GeV}$ & $0.1 \mathrm{GeV}$ & $0.9 \mathrm{GeV}$ & $0.2 \mathrm{GeV}$ \\
\hline background model & $1.3 \mathrm{GeV}$ & $0.2 \mathrm{GeV}$ & $0.2 \mathrm{GeV}$ & $0.2 \mathrm{GeV}$ \\
\hline fake background model & $0.2 \mathrm{GeV}$ & $0.1 \mathrm{GeV}$ & $0.2 \mathrm{GeV}$ & $0.1 \mathrm{GeV}$ \\
\hline template statistics & $0.6 \mathrm{GeV}$ & $0.03 \mathrm{GeV}$ & $0.2 \mathrm{GeV}$ & $0.1 \mathrm{GeV}$ \\
\hline ensemble statistics & $0.1 \mathrm{GeV}$ & $0.1 \mathrm{GeV}$ & $0.1 \mathrm{GeV}$ & $0.04 \mathrm{GeV}$ \\
\hline total systematic error & $2.5 \mathrm{GeV}$ & $2.0 \mathrm{GeV}$ & $2.4 \mathrm{GeV}$ & $1.8 \mathrm{GeV}$ \\
\hline expected statistical error & $7.5 \mathrm{GeV}$ & $3.4 \mathrm{GeV}$ & $8.7 \mathrm{GeV}$ & $3.0 \mathrm{GeV}$ \\
\hline observed statistical error & $8.1 \mathrm{GeV}$ & $3.3 \mathrm{GeV}$ & $13.2 \mathrm{GeV}$ & $3.0 \mathrm{GeV}$ \\
\hline total expectec & $7.9 \mathrm{GeV}$ & $3.9 \mathrm{GeV}$ & $9.0 \mathrm{GeV}$ & $3.5 \mathrm{GeV}$ \\
\hline total observed & $8.4 \mathrm{GeV}$ & $3.9 \mathrm{GeV}$ & $13.4 \mathrm{GeV}$ & $3.5 \mathrm{GeV}$ \\
\hline
\end{tabular}




\subsection{Combination of Systematic Uncertainties for Run IIa and Run IIb}

Table 8.5: Combination of uncertainties for Run IIa Run IIb.

\begin{tabular}{lllr} 
Source & Run IIa & Run IIb & Combination \\
\hline Jet energy scale (JES) & $1.4 \mathrm{GeV}$ & $1.5 \mathrm{GeV}$ & $1.5 \mathrm{GeV}$ \\
b/light response ratio & $0.5 \mathrm{GeV}$ & $0.6 \mathrm{GeV}$ & $0.5 \mathrm{GeV}$ \\
Sample dependent JES & $0.3 \mathrm{GeV}$ & $0.2 \mathrm{GeV}$ & $0.2 \mathrm{GeV}$ \\
b fragmentation & $0.4 \mathrm{GeV}$ & $0.0 \mathrm{GeV}$ & $0.1 \mathrm{GeV}$ \\
Event generator & $0.7 \mathrm{GeV}$ & $0.8 \mathrm{GeV}$ & $0.8 \mathrm{GeV}$ \\
Extra jets modeling & $0.2 \mathrm{GeV}$ & $0.2 \mathrm{GeV}$ & $0.2 \mathrm{GeV}$ \\
PDF variation & $0.4 \mathrm{GeV}$ & $0.4 \mathrm{GeV}$ & $0.4 \mathrm{GeV}$ \\
Jet energy resolution & $0.1 \mathrm{GeV}$ & $0.0 \mathrm{GeV}$ & $0.1 \mathrm{GeV}$ \\
Jet identification & $0.5 \mathrm{GeV}$ & $0.0 \mathrm{GeV}$ & $0.2 \mathrm{GeV}$ \\
Muon/track pT resolution & $0.1 \mathrm{GeV}$ & $0.0 \mathrm{GeV}$ & $0.1 \mathrm{GeV}$ \\
Electron energy resolution & $0.2 \mathrm{GeV}$ & $0.2 \mathrm{GeV}$ & $0.2 \mathrm{GeV}$ \\
MC corrections & $0.2 \mathrm{GeV}$ & $0.0 \mathrm{GeV}$ & $0.1 \mathrm{GeV}$ \\
Background yield & $0.1 \mathrm{GeV}$ & $0.1 \mathrm{GeV}$ & $0.1 \mathrm{GeV}$ \\
Background template shape & $0.3 \mathrm{GeV}$ & $0.2 \mathrm{GeV}$ & $0.2 \mathrm{GeV}$ \\
Template statistics & $0.7 \mathrm{GeV}$ & $0.1 \mathrm{GeV}$ & $0.2 \mathrm{GeV}$ \\
MC calibration & $0.1 \mathrm{GeV}$ & $0.1 \mathrm{GeV}$ & $0.1 \mathrm{GeV}$ \\
& & & \\
\hline & & & \\
total systematic error & $2.0 \mathrm{GeV}$ & $1.9 \mathrm{GeV}$ & $1.9 \mathrm{GeV}$
\end{tabular}




\section{Chapter 9}

\section{Conclusion}

\subsection{Combination}

We have two measurements from independent data sets, but with systematic errors that are mostly correlated between those data sets. As spelled out in Appendix A, we can use the Best Linear Unbiased Estimator (BLUE) method to extract an unbiased combination of the two measurements. We assume that most of our systematic errors are $100 \%$ correlated between the two data-sets, the exceptions to this are the template and ensemble statistics systematics, which are not regarded as correlated between data-sets.

The combined result for the MWT method in the full Run II data-set is:

$$
m_{t}=174.3 \pm 2.6(\text { stat }) \pm 1.8(\text { stat }) \mathrm{GeV}
$$

The precision of this measurement can be improved slightly by adding the NuWT result from Run IIa[63]:

$$
m_{t}=174.7 \pm 2.5(\text { stat }) \pm 1.8(\text { syst }) \mathrm{GeV} .
$$

This can then be combined with the Run I dilepton result, and the lepton+jet results from Run I and Run II, to produce a combined result for the DØ experiment of[64]:

$$
m_{t}=174.4 \pm 0.8(\text { stat }) \pm 1.5(\text { syst }) \mathrm{GeV}
$$




\subsection{Comparison with Other Measurements, Impact}

The current Tevatron combination for the top quark mass is[13] (see Fig. 9.1):

$$
m_{t}=173.1 \pm 0.6(\text { stat }) \pm 1.1(\text { syst }) \mathrm{GeV} .
$$

Our result in this thesis is consistent with the current Tevatron combination and the current $\mathrm{D} \varnothing$ combination. The result in Run IIa is comparable to the result in RunIIb, the result in the dilepton channels in consistent with the result in the lepton+jets channels. The results for the different channels within the dilepton channels are consistent within their uncertainties.

The measurement of the top mass in the dilepton channel has smaller statistics and less visible kinematic information than the lepton+jets or all-jets channels. However, it should be pointed out that the precision of the dilepton channel alone has surpassed that of the combined Run I result [65], and with $4 \mathrm{fb}^{-1}$ of data our statistical error is approaching the size of our systematic error.

\subsection{Prospects for Top Mass at the Tevatron}

The current measurement of the top mass at the Tevatron is dominated by the systematic uncertainty. Any hope of improvement would require an improvement in the systematic error, and this can only come from a better understanding of the detector and our calibrations and resolutions, and a better understanding of the theoretical uncertainties. 


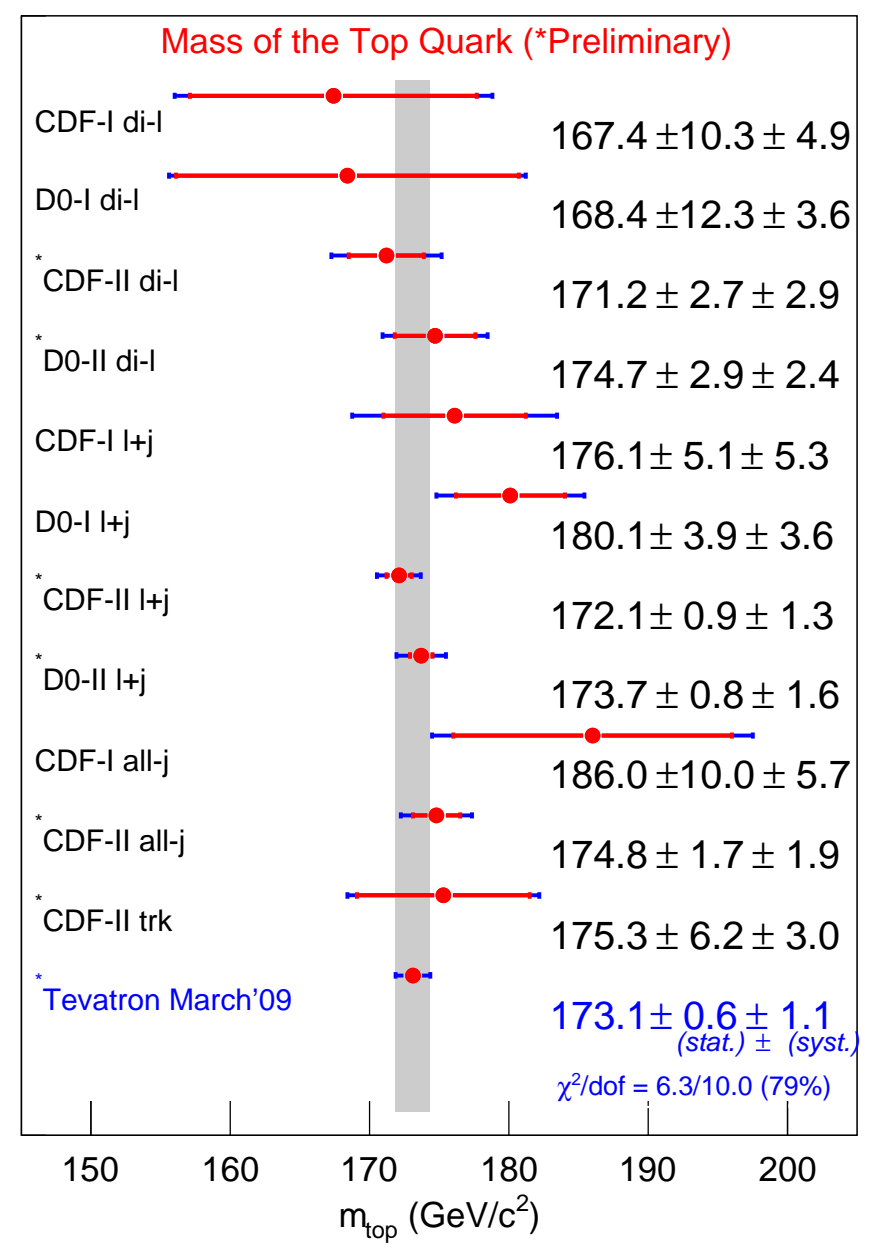

Figure 9.1: The world average top mass, along with the contributing measurements form $\mathrm{D} \varnothing$ and $\mathrm{CDF}[13]$. 


\section{Appendix A}

\section{Combining Measurements with}

\section{Correlated Errors: BLUE Method}

Consider a set of $N$ measurements of a particular quantity, $m_{t}^{i}$, where each measurement has a statistical error, $\delta m_{t}^{i, s t a t}$, and various sources of systematic error, $\delta m_{t}^{i, s y s t_{j}}$. The statistical errors should be uncorrelated, but may not be, and the various systematics errors may or may not be correlated between measurements. Assume that the errors are all gaussian and symmetric, and assigning a correlation matrix to the statistical $\left(\rho_{i j}^{\text {stat }}\right)$, and each systematic error $\left(\rho_{i j}^{s y s t_{k}}\right)$. The best unbiased combined measurement can be found by a weighted average of the individual measurements [66]:

$$
\begin{aligned}
m_{t}^{\text {comb }} & =\sum_{i} w_{i} m_{t}^{i}, \\
\delta m_{t}^{\text {comb }, \text { stat }} & =\sum_{i j} \rho_{i j}^{\text {stat }} \delta m_{t}^{i, \text { stat }} \delta m_{t}^{j, s t a t}, \\
\delta m_{t}^{\text {comb }, \text { syst } t_{k}} & =\sum_{i j} \rho_{i j}^{\text {syst }_{k}} \delta m_{t}^{i, \text { syst }_{k}} \delta m_{t}^{j, \text { syst }_{k}} .
\end{aligned}
$$

Where the weights can be shown to be:

$$
w_{i}=\frac{\sum_{k} \operatorname{Cov}_{i k}^{-1}}{\sum_{j k} \operatorname{Cov}_{j k}^{-1}} .
$$

Where $\operatorname{Cov}^{-1}$ is the inverse of the covariance matrix defined as:

$$
\operatorname{Cov}_{i j}=\rho_{i j}^{s t a t} \delta m_{t}^{i, s t a t} \delta m_{t}^{j, s t a t}+\sum_{s y s t_{k}} \rho_{i j}^{\text {syst }_{k}} \delta m_{t}^{i, s y s t_{k}} \delta m_{t}^{j, s y s t_{k}} .
$$




\section{Appendix B}

\section{Jet Transfer Function Parameters for RunIIb}

The transfer functions $W_{\text {jet }}\left(E_{\text {det }}, E_{\text {part }}\right)$ are the probability of measuring a jet energy $E_{j e t}$, given a parton energy $E_{\text {det }}$.

$$
\begin{aligned}
W_{j e t}\left(E_{\text {det }}, E_{\text {part }}\right) & =\frac{1}{\sqrt{2 \pi}\left(p_{2}+p_{3} p_{4}\right)} \\
& \times\left[\exp \left(-\frac{\left(\left(E_{\text {det }}-E_{\text {part }}\right)-p_{1}\right)^{2}}{2 p_{2}^{2}}\right)+p_{3} \exp \left(-\frac{\left(\left(E_{\text {det }}-E_{\text {part }}\right)-p_{4}\right)^{2}}{2 p_{5}^{2}}\right)\right] .
\end{aligned}
$$

Where each parameter $p_{i}$ is expressed as a function of $E_{\text {part }}$ :

$$
p_{i}=a_{i}+E_{\text {part }} \cdot b_{i}
$$

The transfer functions are derived using PYTHIA signal events in $t \bar{t}$ samples with masses between 155 and $195 \mathrm{GeV}$ in $5 \mathrm{GeV}$ intervals. The functions are split into for regions in pseudorapidity: $|\eta|<0.5,0.5<|\eta|<1.0,1.0<|\eta|<1.5$, and $1.5|\eta|<2.5$, and derived separately for light jets, semileptonic b-jets $b \rightarrow \mu \nu_{\mu}+$ jet, and non-semileptonic b-jets. 


\begin{tabular}{|c||c|c||c|c||c|c|}
\hline \multicolumn{1}{|c||}{$|\eta|<0.5$} & \multicolumn{2}{c||}{$l$-jets } & \multicolumn{2}{c||}{$b$-jets } & \multicolumn{2}{c|}{ slt $b$-jets } \\
\hline$p_{i}=a_{i}+E_{y} \cdot b_{i}$ & $a_{i}$ & $b_{i}$ & $a_{i}$ & $b_{i}$ & $a_{i}$ & $b_{i}$ \\
\hline \hline$p_{1}$ & -2.73604 & 0.01668 & 3.30222 & -0.21335 & 6.36615 & -0.14621 \\
$p_{2}$ & 5.44382 & 0.06292 & 5.01837 & 0.17348 & 2.52583 & 0.14274 \\
$p_{3}$ & 0. & 0.00043 & 0. & 0.03484 & 0. & 0.00039 \\
$p_{4}$ & 15.38106 & -0.21239 & -6.67805 & 0.02378 & 27.98500 & -0.38707 \\
$p_{5}$ & 17.66677 & 0.19570 & 5.06380 & 0.04705 & 18.01274 & 0.13018 \\
\hline
\end{tabular}

\begin{tabular}{|c||c|c||c|c||c|c|}
\hline $0.5 \leq|\eta|<1.0$ & \multicolumn{2}{c||}{$l$-jets } & \multicolumn{2}{c||}{$b$-jets } & \multicolumn{2}{c|}{ slt $b$-jets } \\
\hline$p_{i}=a_{i}+E_{y} \cdot b_{i}$ & $a_{i}$ & $b_{i}$ & $a_{i}$ & $b_{i}$ & $a_{i}$ & $b_{i}$ \\
\hline \hline$p_{1}$ & -0.80216 & -0.00359 & 5.37960 & -0.22568 & 6.30954 & -0.14047 \\
$p_{2}$ & 5.40207 & 0.08462 & 5.08395 & 0.17740 & 3.89121 & 0.13658 \\
$p_{3}$ & 0. & 0.00048 & 0. & 0.02491 & 0. & 0.00034 \\
$p_{4}$ & 20.05600 & -0.23832 & -6.55783 & 0.01905 & 15.16046 & -0.09736 \\
$p_{5}$ & -0.23833 & 18.88547 & 4.36142 & 0.06989 & 23.22244 & 0.02911 \\
\hline
\end{tabular}

\begin{tabular}{|c||c|c||c|c||c|c|}
\hline \multicolumn{1}{|c|}{$1.0 \leq|\eta|<1.5$} & \multicolumn{2}{c||}{$l$-jets } & \multicolumn{2}{c||}{$b$-jets } & \multicolumn{2}{c|}{ slt $b$-jets } \\
\hline$p_{i}=a_{i}+E_{y} \cdot b_{i}$ & $a_{i}$ & $b_{i}$ & $a_{i}$ & $b_{i}$ & $a_{i}$ & $b_{i}$ \\
\hline \hline$p_{1}$ & 0.16920 & 13.22507 & 2.84938 & -0.18472 & 8.00016 & -0.13930 \\
$p_{2}$ & -0.32605 & 6.97212 & 0.97752 & 0.18273 & 8.54300 & 0.12847 \\
$p_{3}$ & 0. & 0.02520 & 0. & 0.00669 & 0. & 0.00019 \\
$p_{4}$ & 4.71037 & -0.00837 & 0.85367 & -0.02829 & 78.91102 & 0.22220 \\
$p_{5}$ & 10.33000 & 0.06417 & 13.76236 & 0.06036 & 27.95641 & -0.28705 \\
\hline
\end{tabular}

\begin{tabular}{|c||c|c||c|c||c|c|}
\hline \multicolumn{1}{|c||}{$1.5 \leq|\eta|$} & \multicolumn{2}{c||}{$l$-jets } & \multicolumn{2}{c||}{$b$-jets } & \multicolumn{2}{c|}{ slt $b$-jets } \\
\hline$p_{i}=a_{i}+E_{y} \cdot b_{i}$ & $a_{i}$ & $b_{i}$ & $a_{i}$ & $b_{i}$ & $a_{i}$ & $b_{i}$ \\
\hline \hline$p_{1}$ & 15.19742 & -0.21723 & 13.76236 & -0.28998 & 16.48620 & -0.19074 \\
$p_{2}$ & 3.34031 & 0.14491 & 3.85718 & 0.13627 & 4.88111 & 0.14323 \\
$p_{3}$ & 0. & 0.00406 & 0. & 0.00752 & 0. & 0.00012 \\
$p_{4}$ & 17.15661 & -0.03689 & 5.59329 & -0.04538 & 47.28978 & 0.05208 \\
$p_{5}$ & 17.50263 & 0.05337 & 15.00816 & 0.07604 & 28.31214 & -0.08554 \\
\hline
\end{tabular}

Figure B.1: Jet Transfer Function parameters in RunIIb. 


\section{Appendix C}

\section{Control Plots for $e \mu$, ee and $\mu \mu$ channels in RunIIb}
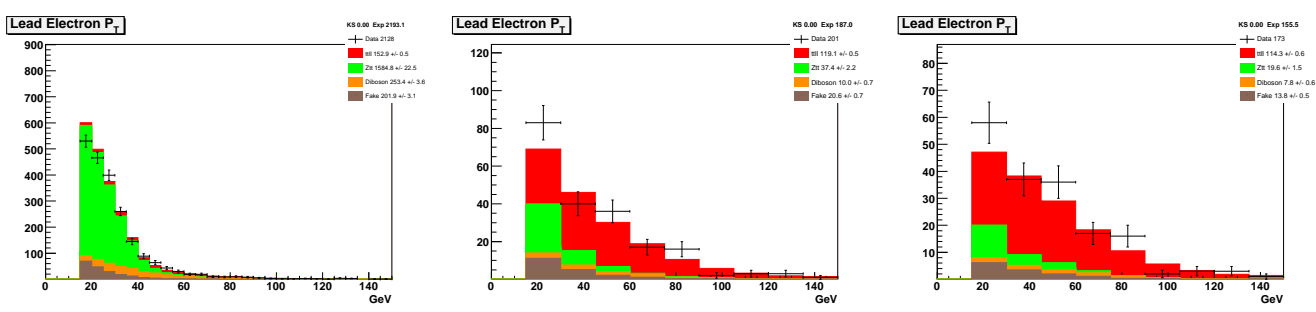

Figure C.1: Electron $p_{T}$ distribution for inclusive jet selection, $\geq 2$ jet selection, and final selection for the $e \mu$ channel.
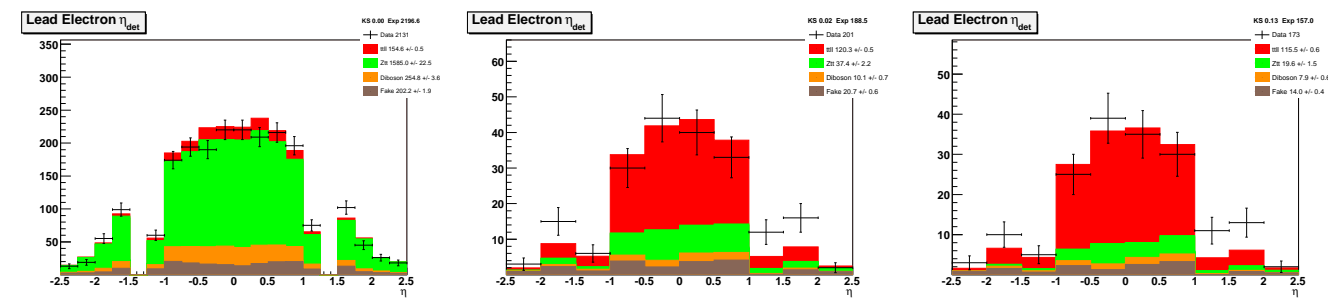

Figure C.2: Electron $\eta_{\text {det }}$ distribution for inclusive jet selection, $\geq 2$ jet selection, and final selection for the $e \mu$ channel. 

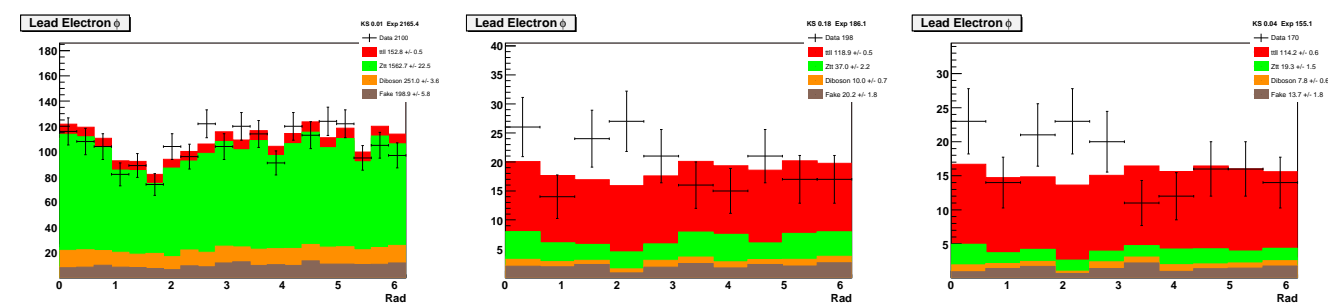

Figure C.3: Electron $\phi$ distribution for inclusive jet selection, $\geq 2$ jet selection, and final selection for the $e \mu$ channel.
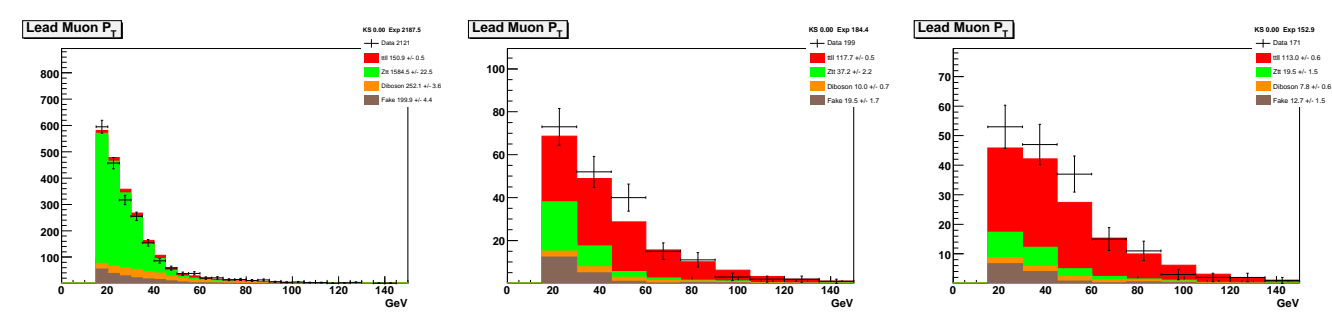

Figure C.4: Muon $p_{T}$ distribution for inclusive jet selection, $\geq 2$ jet selection, and final selection for the $e \mu$ channel.
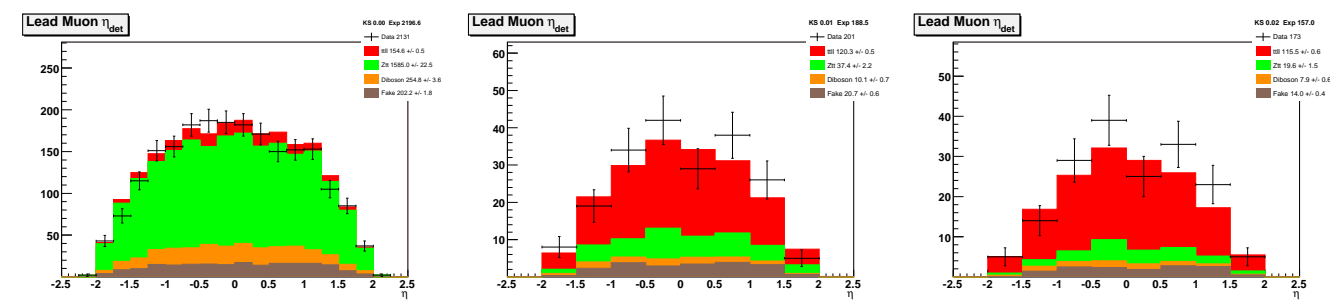

Figure C.5: Muon $\eta_{\text {det }}$ distribution for inclusive jet selection, $\geq 2$ jet selection, and final selection for the $e \mu$ channel.
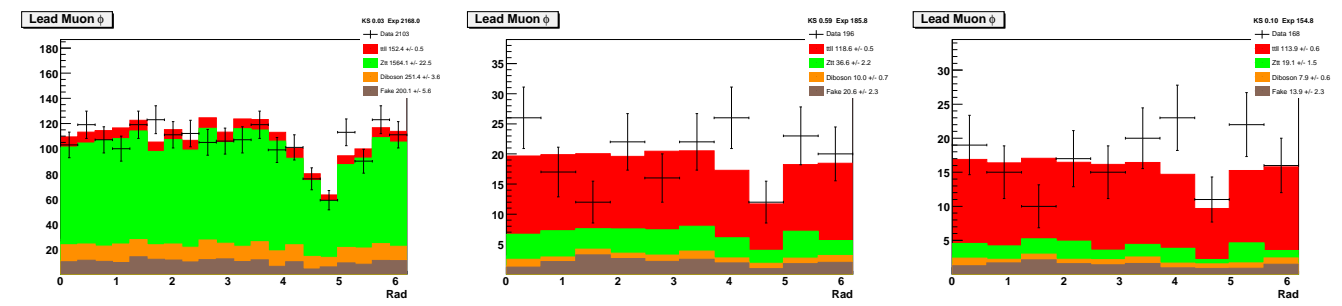

Figure C.6: Muon $\phi$ distribution for inclusive jet selection, $\geq 2$ jet selection, and final selection for the $e \mu$ channel. 

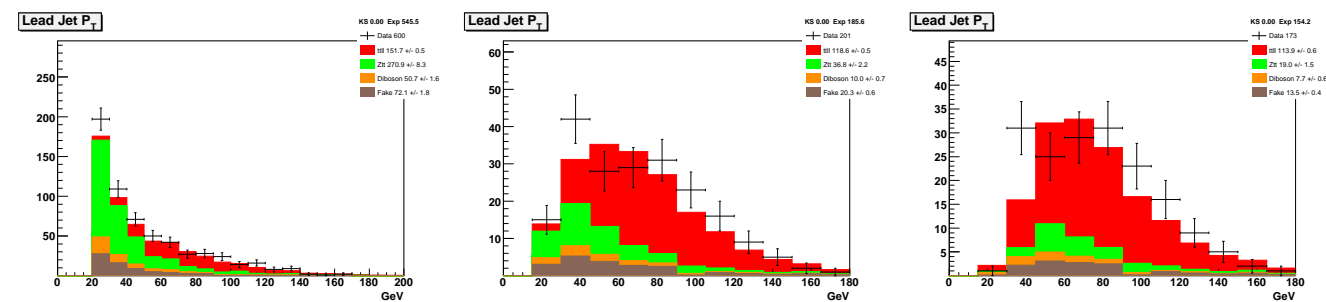

Figure C.7: Leading Jet $p_{T}$ distribution for inclusive jet selection, $\geq 2$ jet selection, and final selection for the $e \mu$ channel.
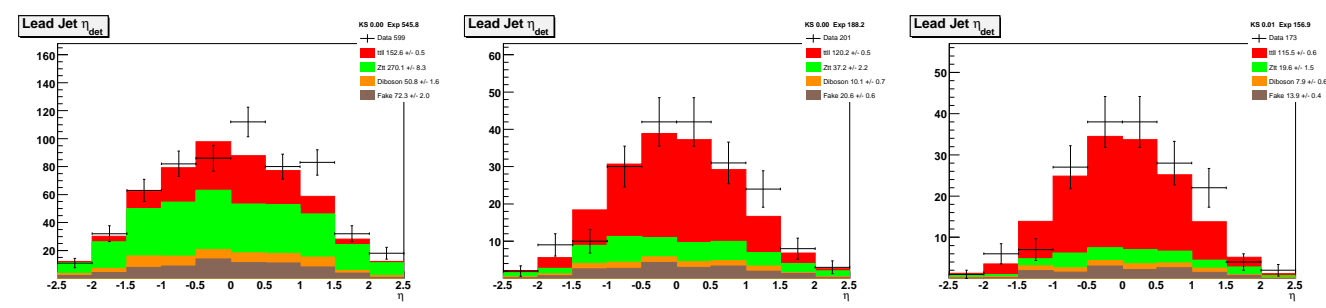

Figure C.8: Leading Jet $\eta_{\text {det }}$ distribution for inclusive jet selection, $\geq 2$ jet selection, and final selection for the $e \mu$ channel.
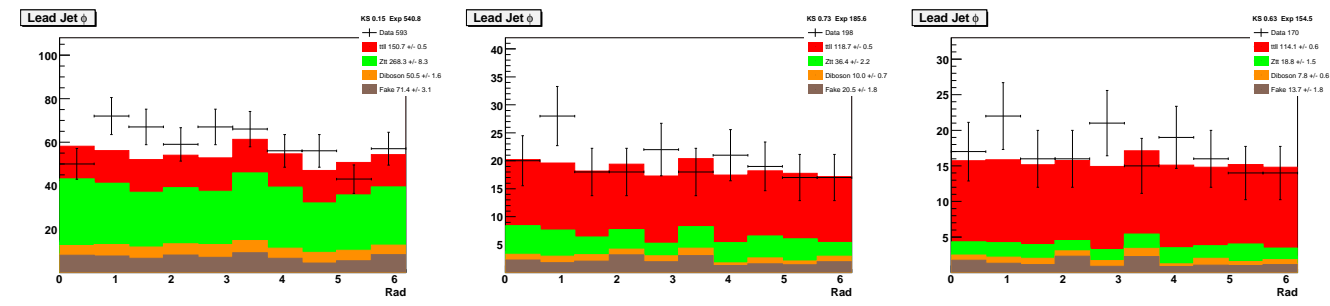

Figure C.9: Leading Jet $\phi$ distribution for inclusive jet selection, $\geq 2$ jet selection, and final selection for the $e \mu$ channel.
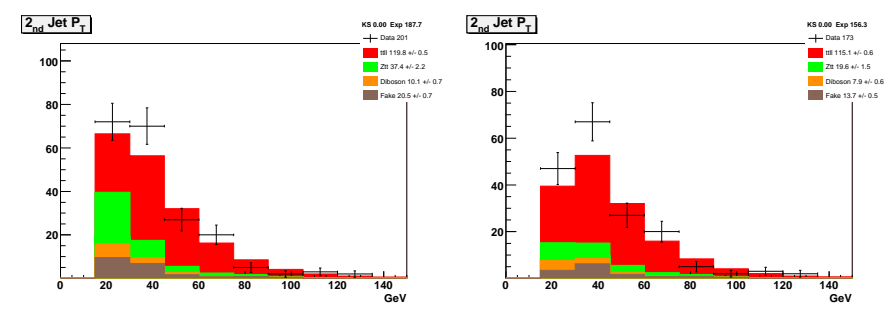

Figure C.10: Second Jet $p_{T}$ distribution for $\geq 2$ jet selection, and final selection for the $e \mu$ channel. 

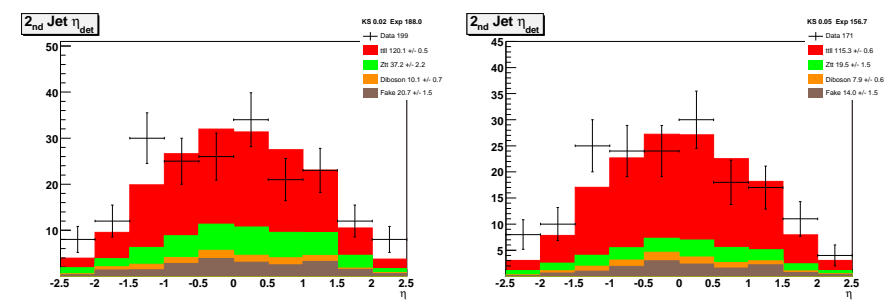

Figure C.11: Second Jet $\eta_{\text {det }}$ distribution for $\geq 2$ jet selection, and final selection for the $e \mu$ channel.
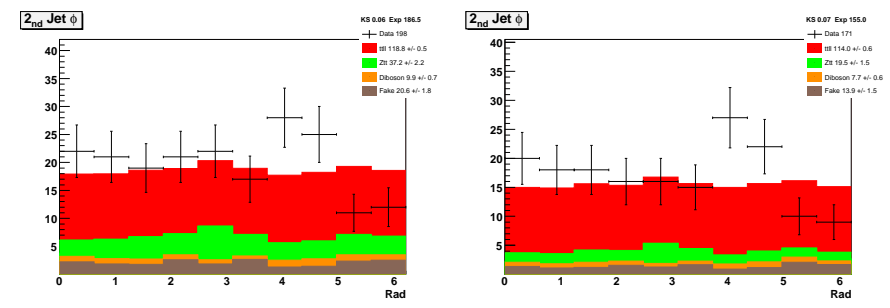

Figure C.12: Second Jet $\phi$ distribution for $\geq 2$ jet selection, and final selection for the $e \mu$ channel.
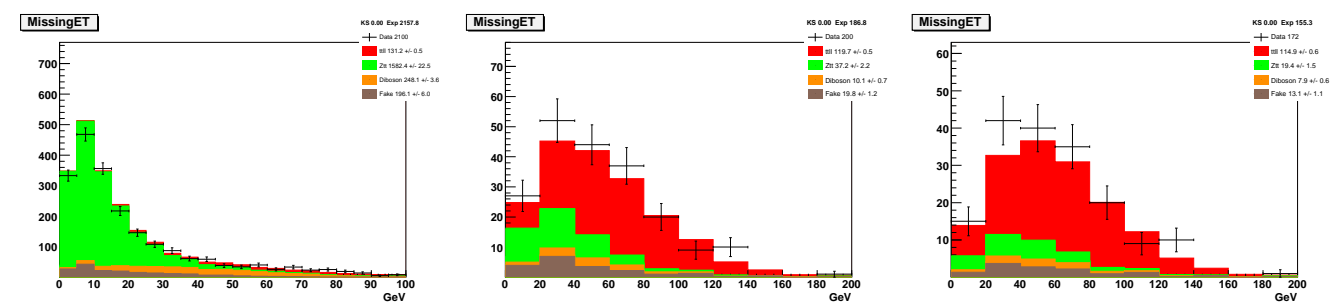

Figure C.13: $\not \not_{T}$ distribution for inclusive jet selection, $\geq 2$ jet selection, and final selection for the $e \mu$ channel.
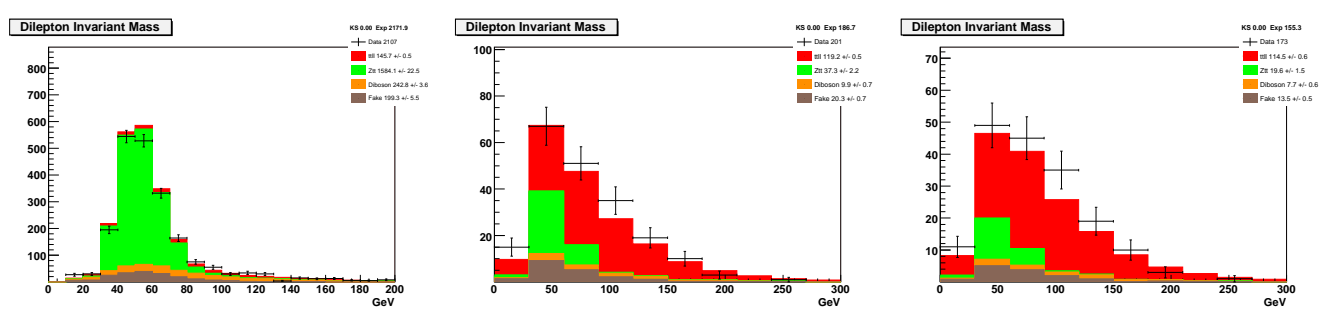

Figure C.14: $m_{e m u}$ distribution for inclusive jet selection, $\geq 2$ jet selection, and final selection for the $e \mu$ channel. 

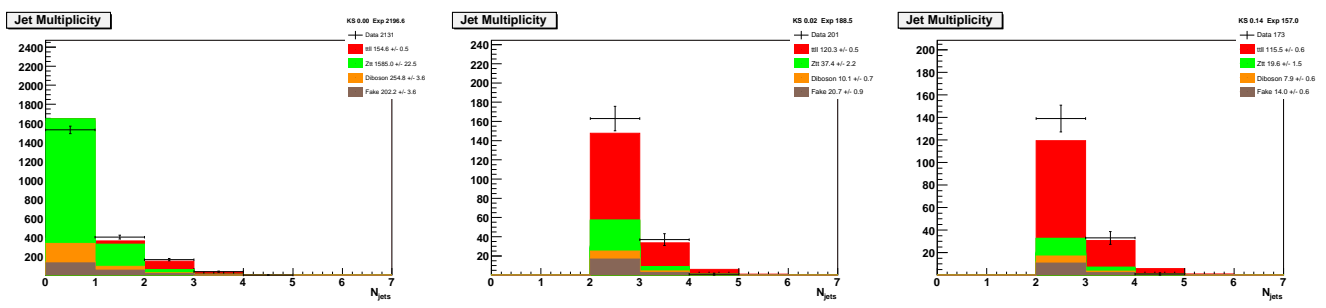

Figure C.15: $N_{\text {jets }}$ distribution for inclusive jet selection, $\geq 2$ jet selection, and final selection for the $e \mu$ channel. 

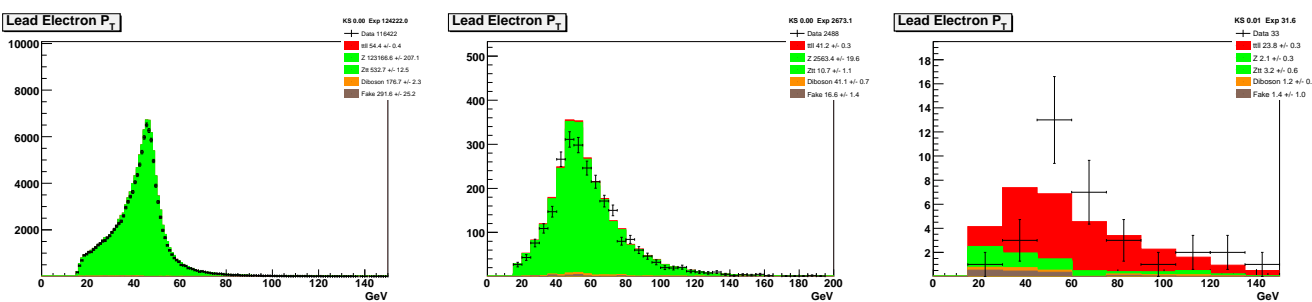

Figure C.16: Leading Electron $p_{T}$ distribution for inclusive jet selection, $\geq 2$ jet selection, and final selection for the ee channel.
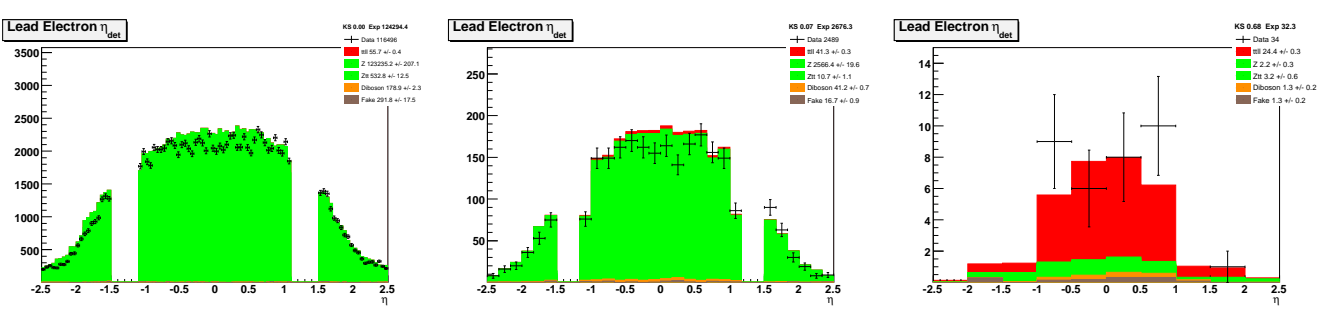

Figure C.17: Leading Electron $\eta_{\text {det }}$ distribution for inclusive jet selection, $\geq 2$ jet selection, and final selection for the ee channel.
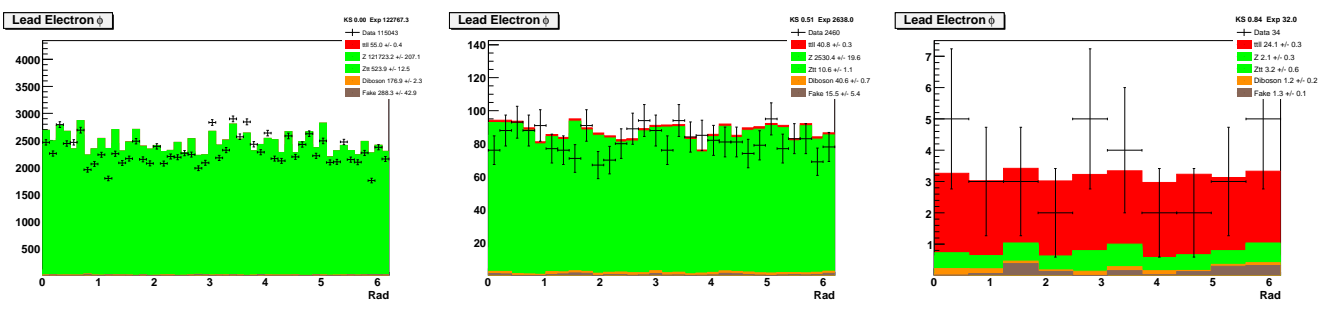

Figure C.18: Leading Electron $\phi$ distribution for inclusive jet selection, $\geq 2$ jet selection, and final selection for the ee channel.
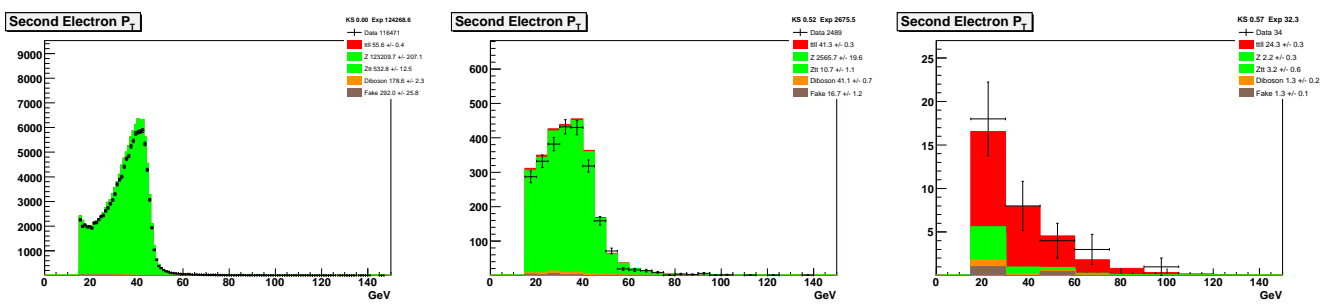

Figure C.19: Second Electron $p_{T}$ distribution for inclusive jet selection, $\geq 2$ jet selection, and final selection for the ee channel. 

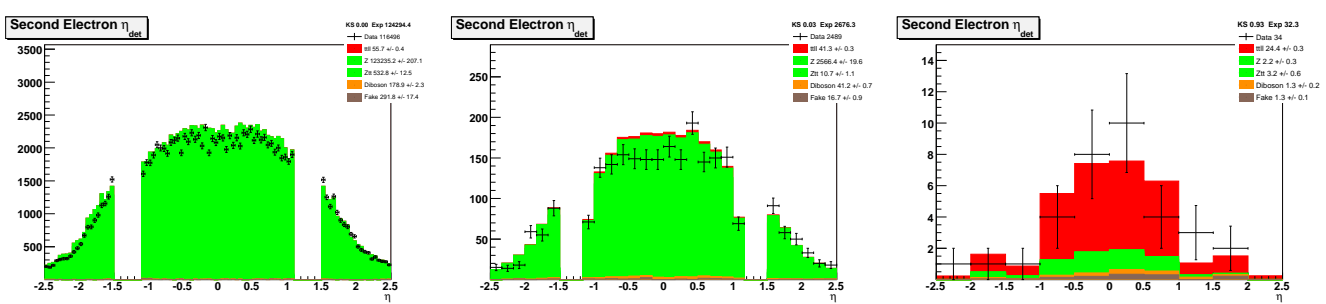

Figure C.20: Second Electron $\eta_{\text {det }}$ distribution for inclusive jet selection, $\geq 2$ jet selection, and final selection for the ee channel.
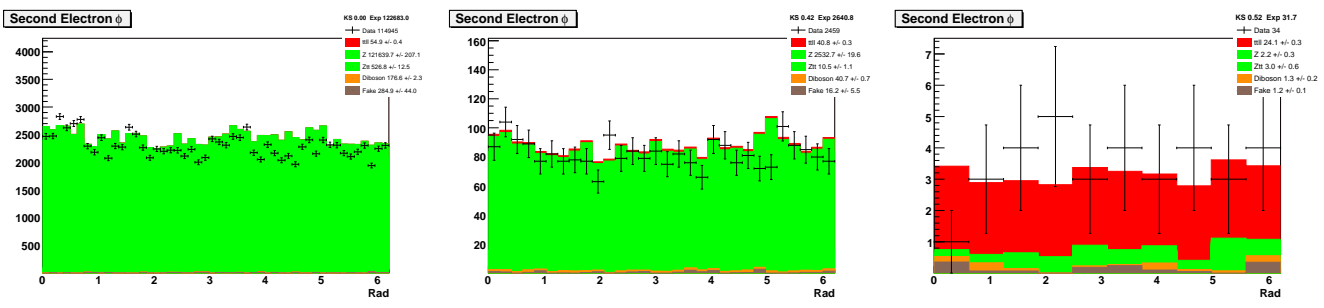

Figure C.21: Second Electron $\phi$ distribution for inclusive jet selection, $\geq 2$ jet selection, and final selection for the ee channel.
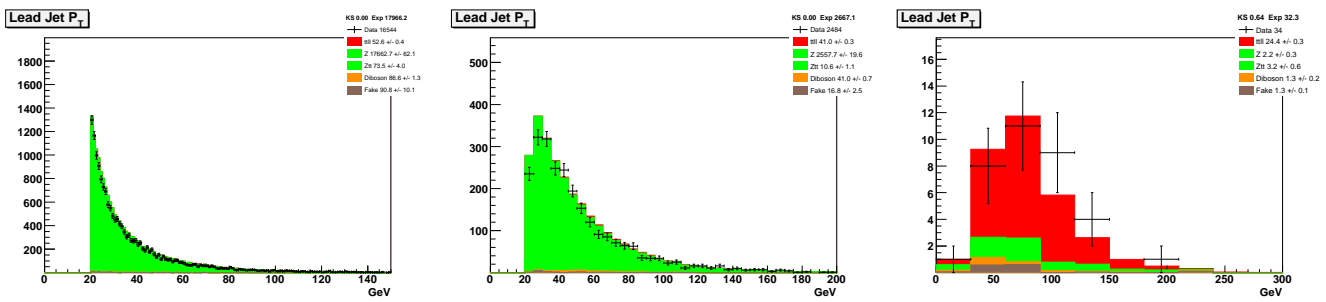

Figure C.22: Leading Jet $p_{T}$ distribution for inclusive jet selection, $\geq 2$ jet selection, and final selection for the ee channel.
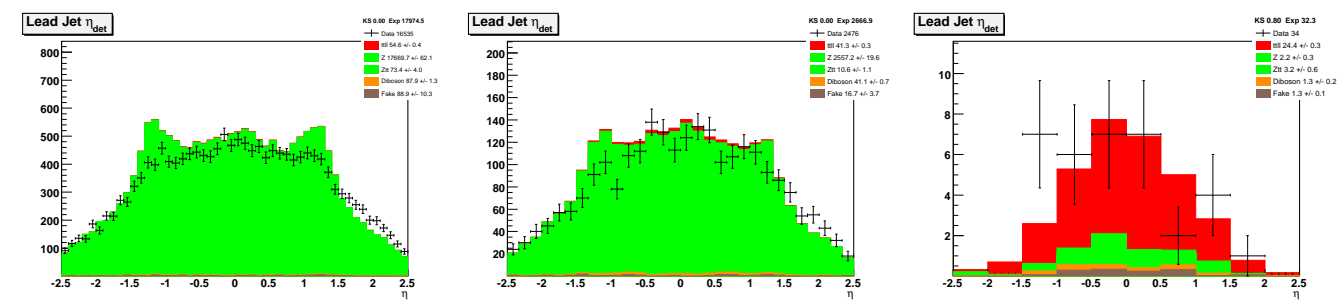

Figure C.23: Leading Jet $\eta_{\text {det }}$ distribution for inclusive jet selection, $\geq 2$ jet selection, and final selection for the ee channel. 

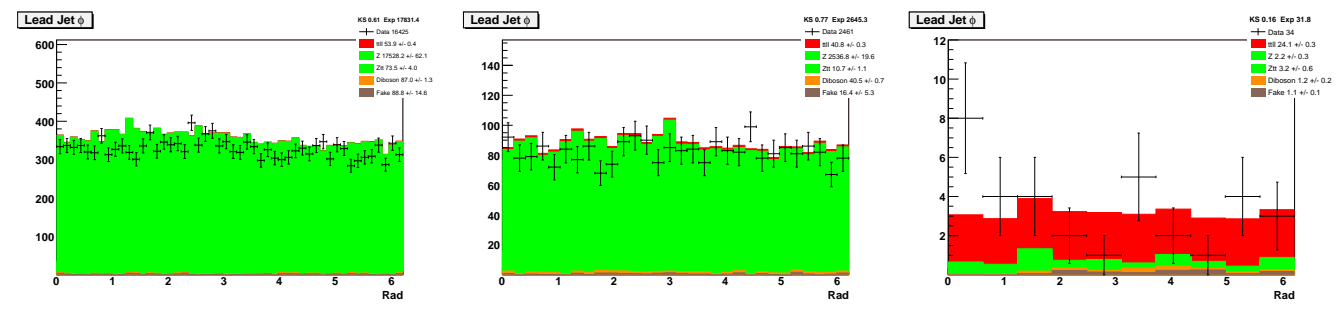

Figure C.24: Leading Jet $\phi$ distribution for inclusive jet selection, $\geq 2$ jet selection, and final selection for the ee channel.
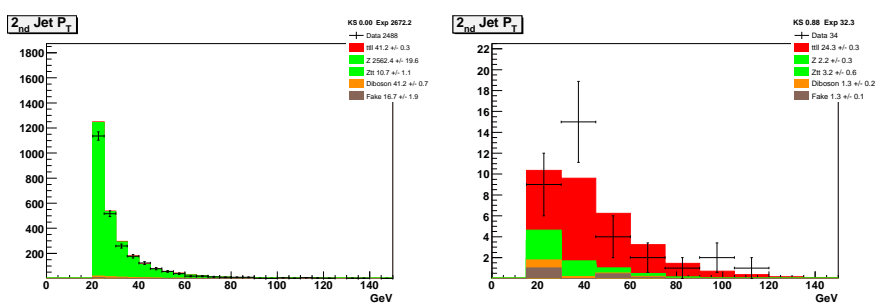

Figure C.25: Second Jet $p_{T}$ distribution for $\geq 2$ jet selection, and final selection for the $e e$ channel.
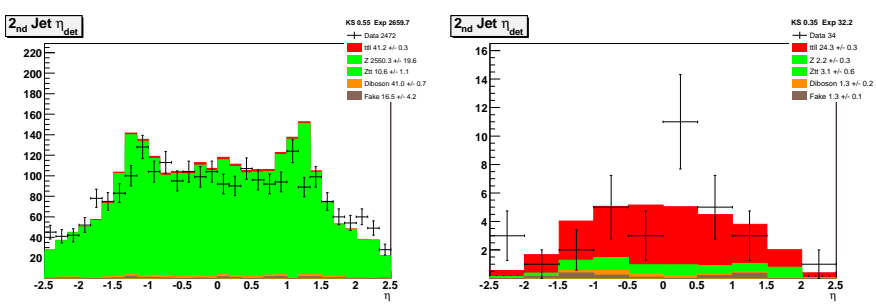

Figure C.26: Second Jet $\eta_{\text {det }}$ distribution for $\geq 2$ jet selection, and final selection for the ee channel.
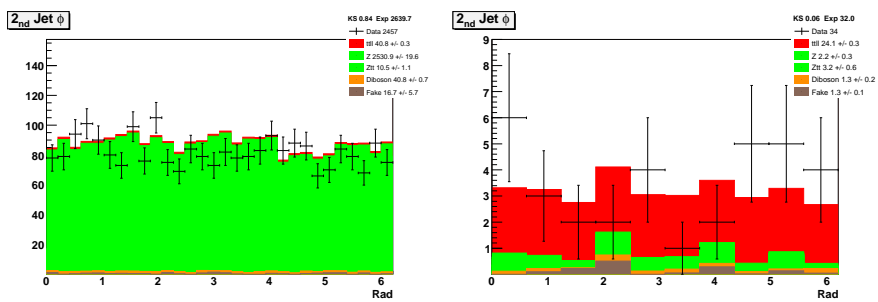

Figure C.27: Second Jet $\phi$ distribution for $\geq 2$ jet selection, and final selection for the $e e$ channel. 

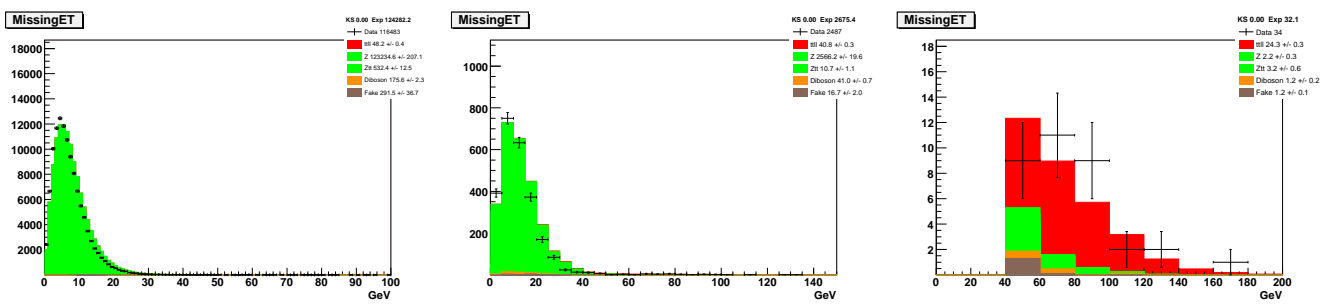

Figure C.28: $\not p_{T}$ distribution for inclusive jet selection, $\geq 2$ jet selection, and final selection for the ee channel.
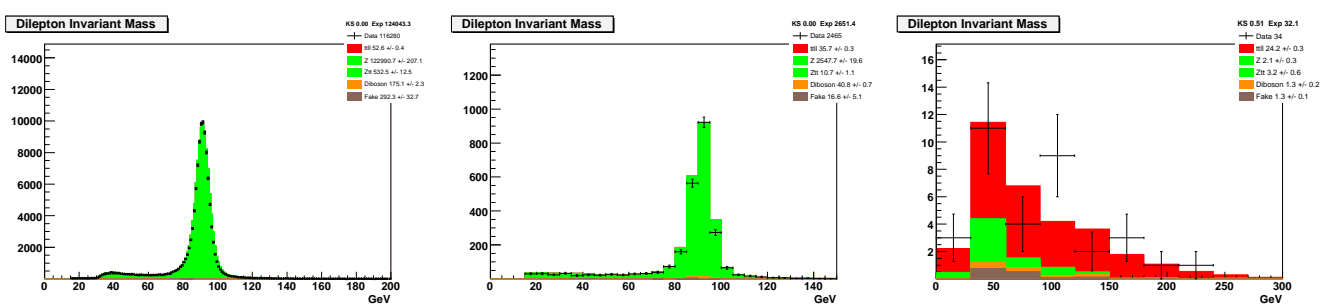

Figure C.29: $m_{e e}$ distribution for inclusive jet selection, $\geq 2$ jet selection, and final selection for the ee channel.
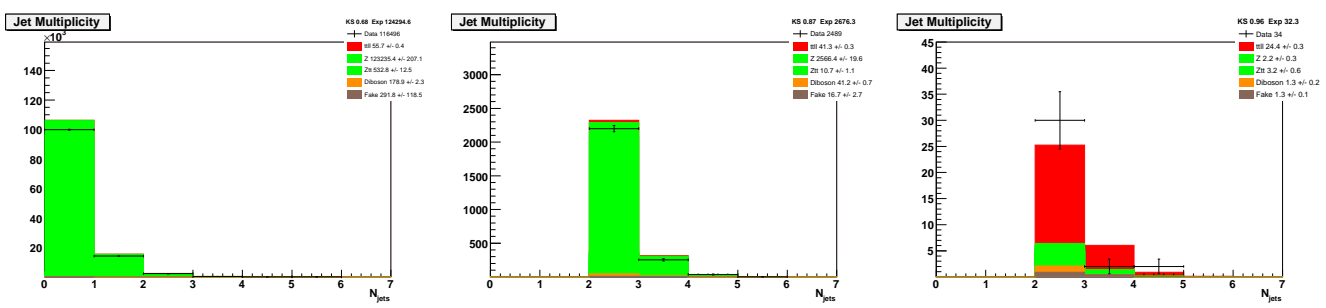

Figure C.30: $N_{\text {jets }}$ distribution for inclusive jet selection, $\geq 2$ jet selection, and final selection for the ee channel. 

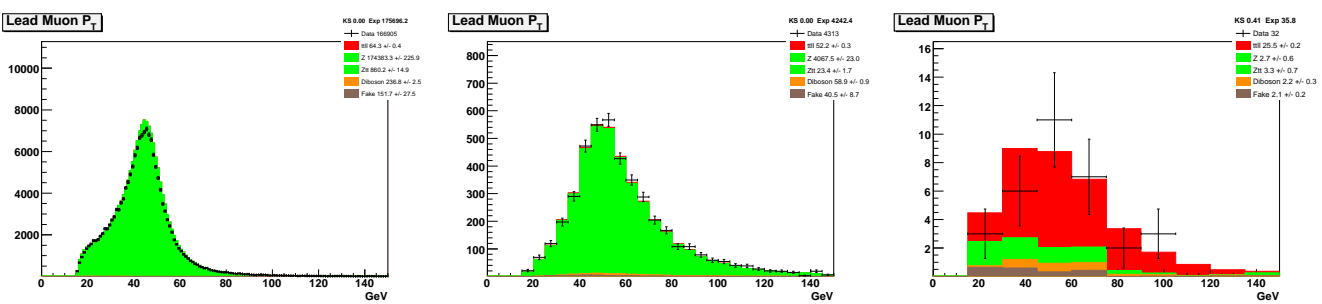

Figure C.31: Leading Muon $p_{T}$ distribution for inclusive jet selection, $\geq 2$ jet selection, and final selection for the $\mu \mu$ channel.
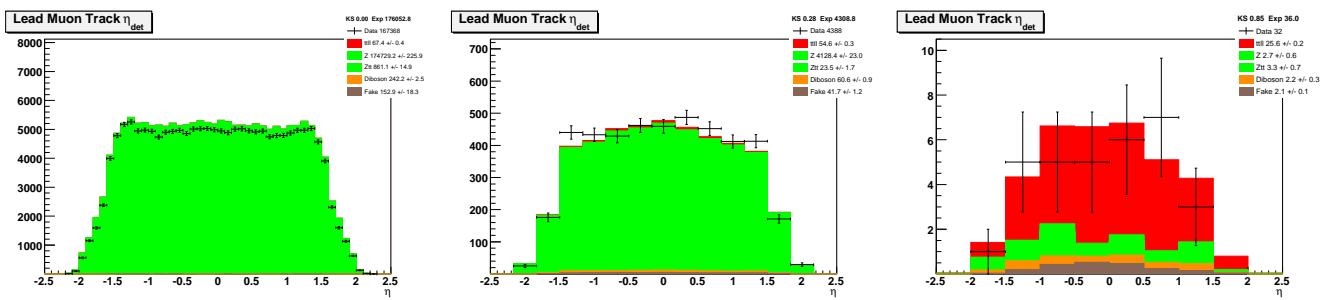

Figure C.32: Leading Muon $\eta_{\text {det }}$ distribution for inclusive jet selection, $\geq 2$ jet selection, and final selection for the $\mu \mu$ channel.
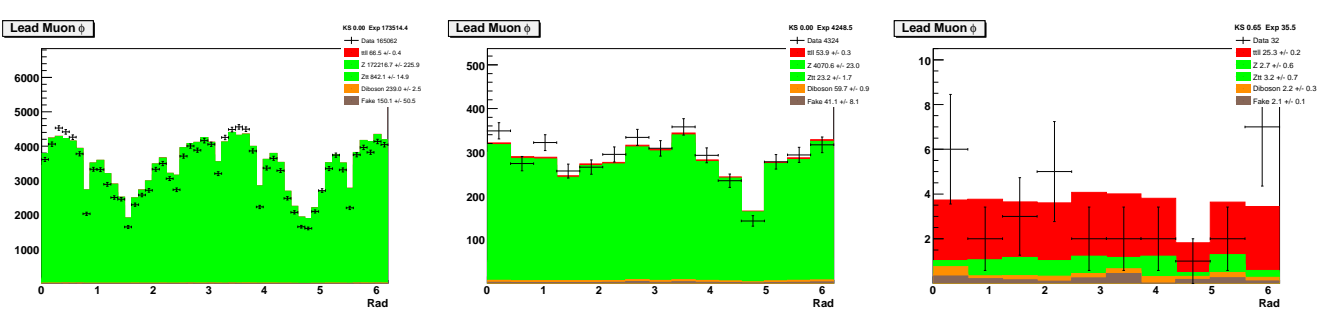

Figure C.33: Leading Muon $\phi$ distribution for inclusive jet selection, $\geq 2$ jet selection, and final selection for the $\mu \mu$ channel.
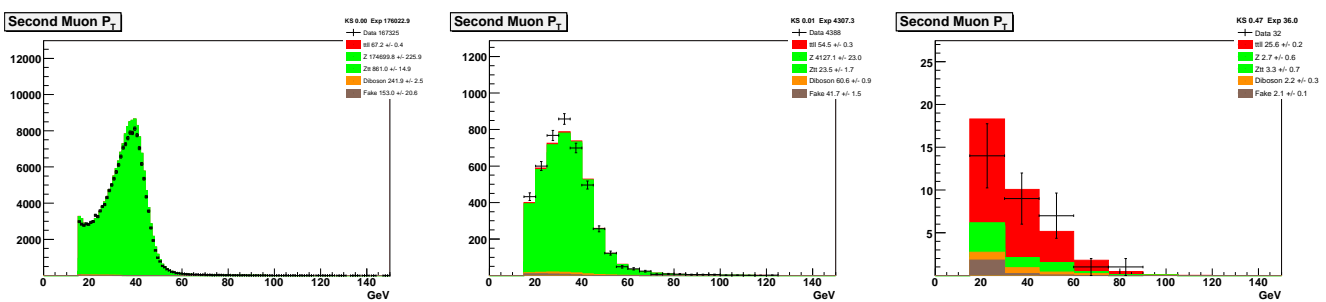

Figure C.34: Second Muon $p_{T}$ distribution for inclusive jet selection, $\geq 2$ jet selection, and final selection for the $\mu \mu$ channel. 

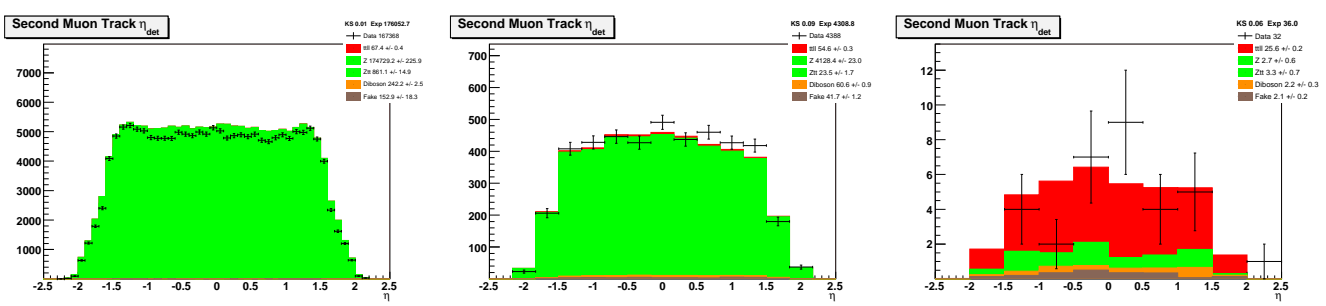

Figure C.35: Second Muon $\eta_{\text {det }}$ distribution for inclusive jet selection, $\geq 2$ jet selection, and final selection for the $\mu \mu$ channel.
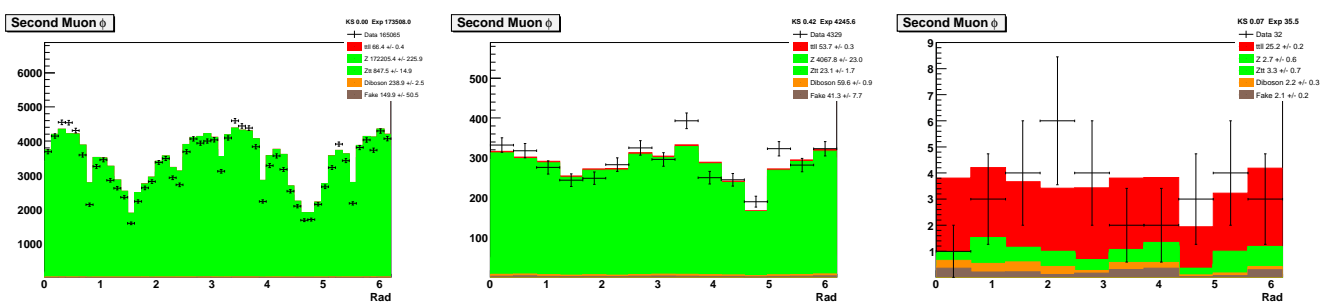

Figure C.36: Second Muon $\phi$ distribution for inclusive jet selection, $\geq 2$ jet selection, and final selection for the $\mu \mu$ channel.
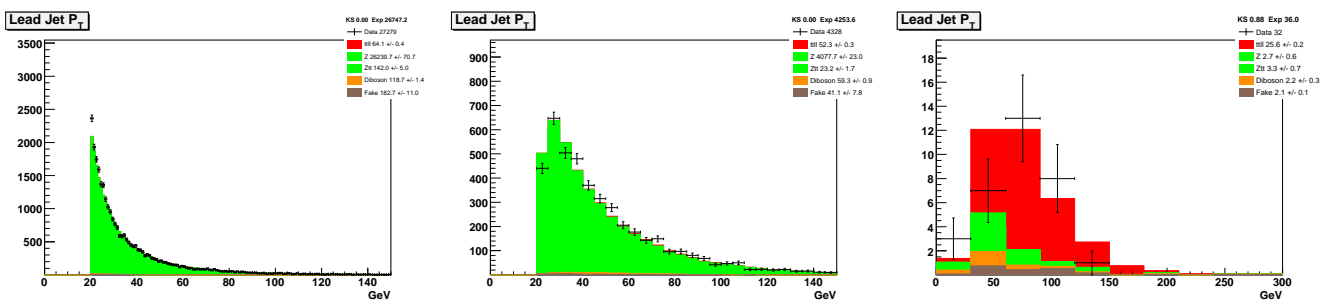

Figure C.37: Leading Jet $p_{T}$ distribution for inclusive jet selection, $\geq 2$ jet selection, and final selection for the $\mu \mu$ channel.
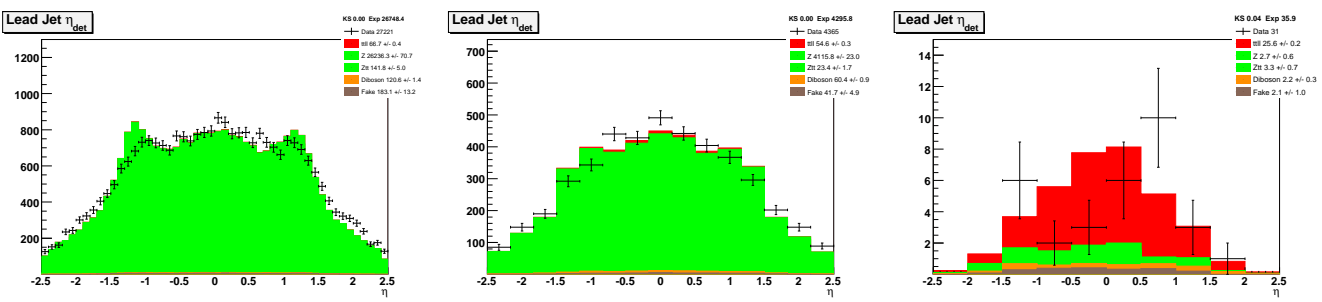

Figure C.38: Leading Jet $\eta_{\text {det }}$ distribution for inclusive jet selection, $\geq 2$ jet selection, and final selection for the $\mu \mu$ channel. 

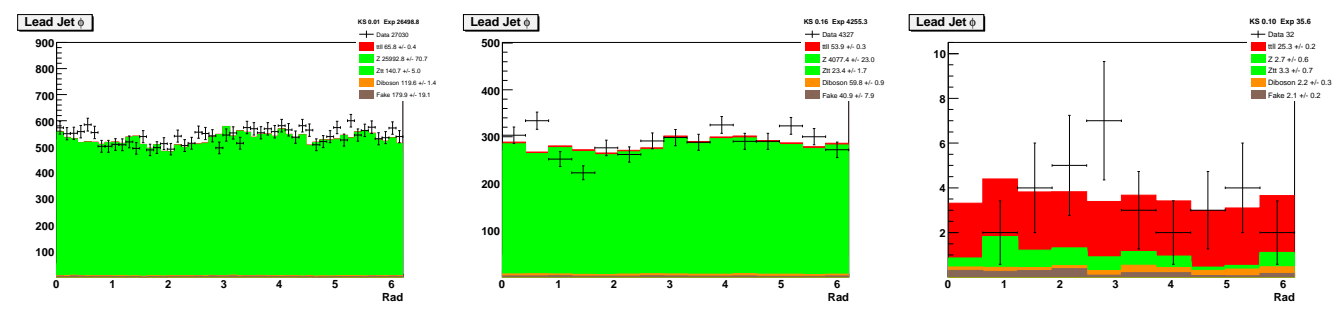

Figure C.39: Leading Jet $\phi$ distribution for inclusive jet selection, $\geq 2$ jet selection, and final selection for the $\mu \mu$ channel.
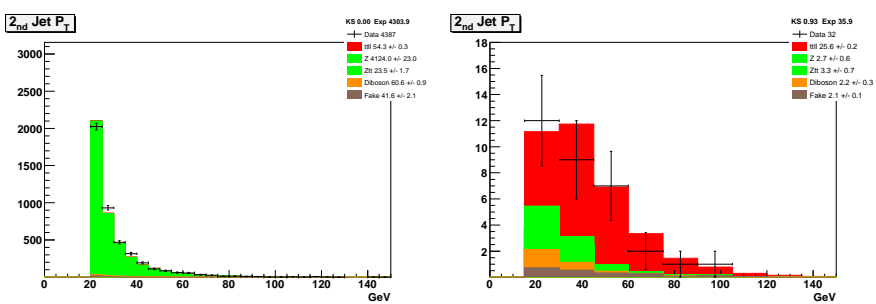

Figure C.40: Second Jet $p_{T}$ distribution for $\geq 2$ jet selection, and final selection for the $\mu \mu$ channel.
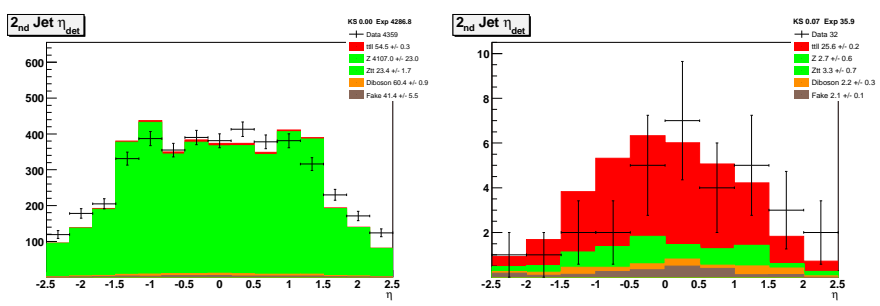

Figure C.41: Second Jet $\eta_{\text {det }}$ distribution for $\geq 2$ jet selection, and final selection for the $\mu \mu$ channel.
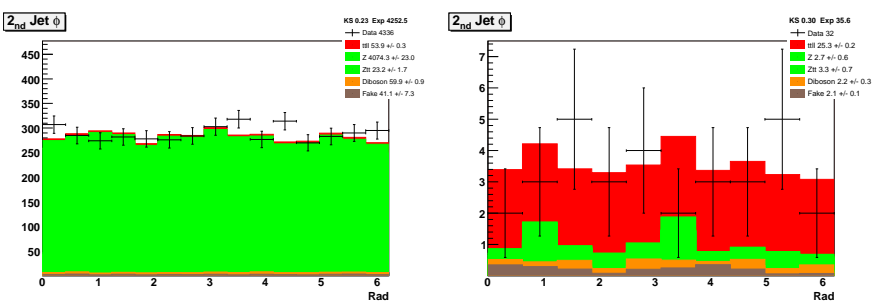

Figure C.42: Second Jet $\phi$ distribution for $\geq 2$ jet selection, and final selection for the $\mu \mu$ channel. 

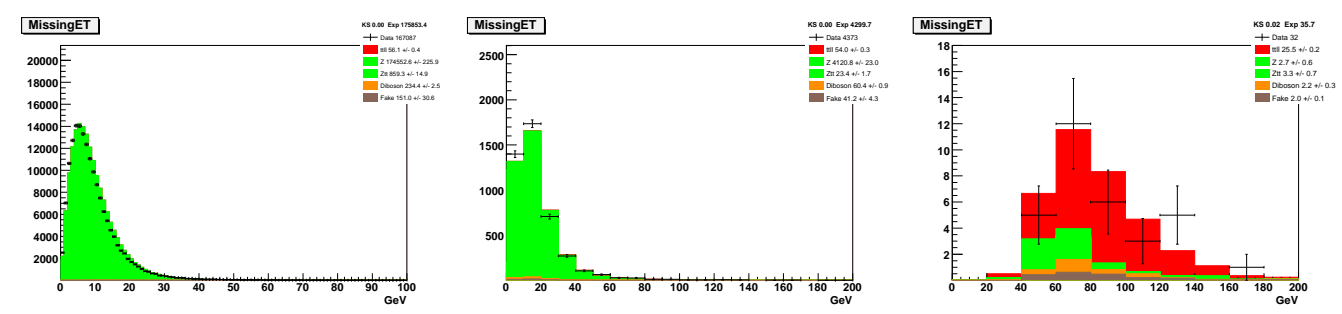

Figure C.43: $\not{ }_{T}^{\prime}$ distribution for inclusive jet selection, $\geq 2$ jet selection, and final selection for the $\mu \mu$ channel.
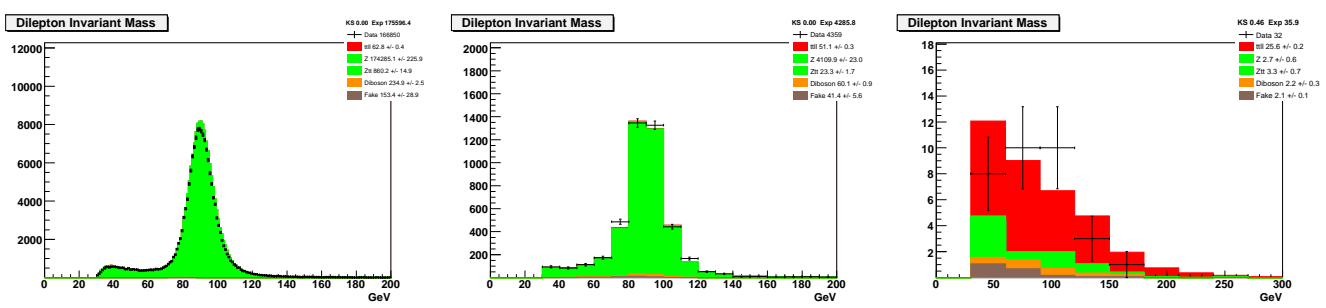

Figure C.44: $m_{\mu \mu}$ distribution for inclusive jet selection, $\geq 2$ jet selection, and final selection for the $\mu \mu$ channel.
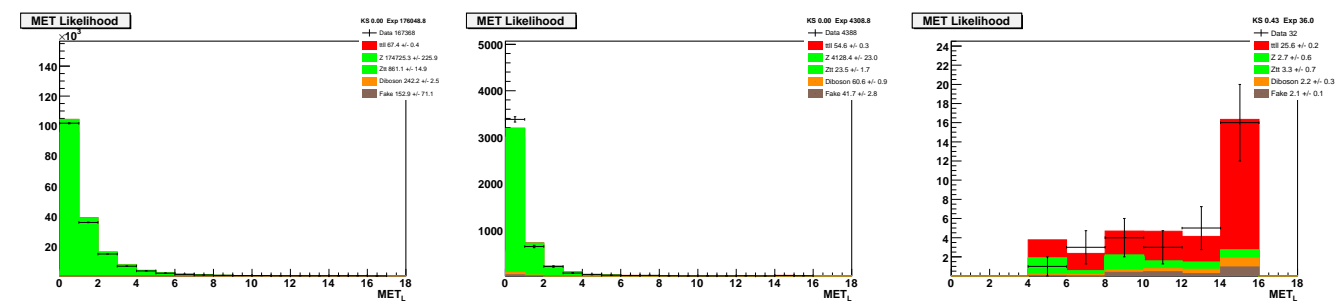

Figure C.45: $p_{T}^{\sigma}$ distribution for inclusive jet selection, $\geq 2$ jet selection, and final selection for the $\mu \mu$ channel.
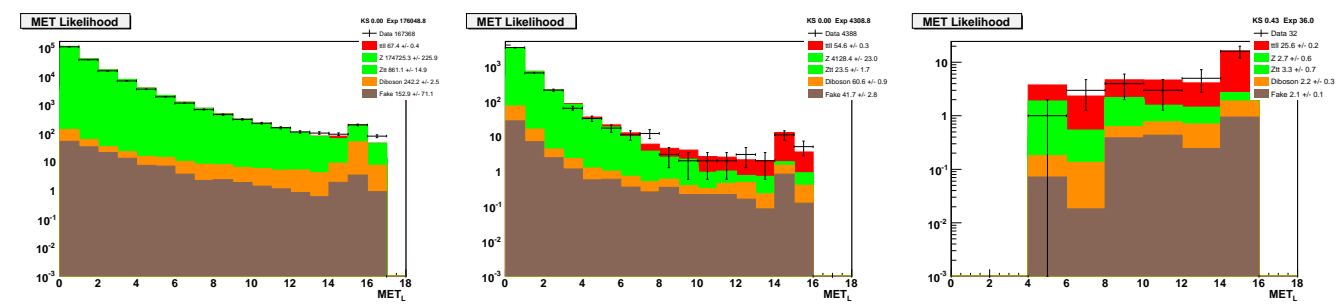

Figure C.46: $p_{T}^{\sigma}$ distribution for inclusive jet selection, and $\geq 2$ jet selection, in Log scale. 

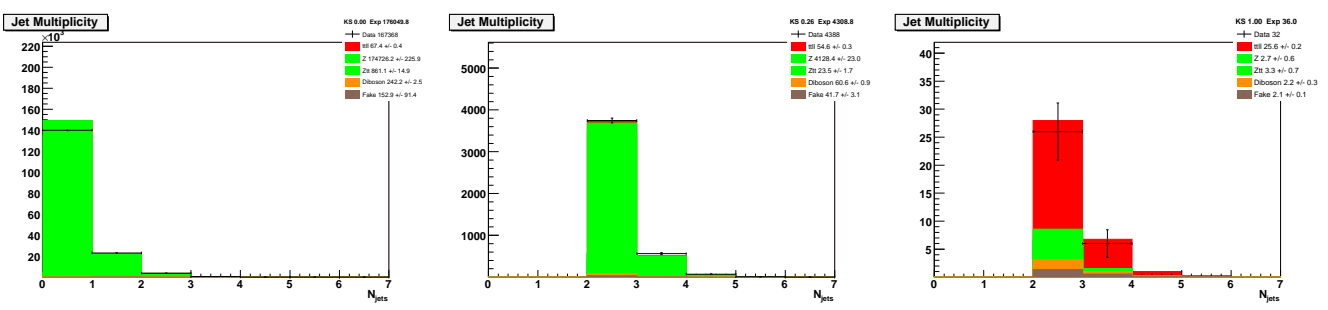

Figure C.47: $N_{\text {jets }}$ distribution for inclusive jet selection, $\geq 2$ jet selection, and final selection for the $\mu \mu$ channel. 


\section{List of Journal Abbreviations}

Comput. Phys. Commun. . . . . . . . . Computer Physics Communications

Eur. Phys. J. . . . . . . . . . . . . . The European Physical Journal JHEP . . . . . . . . . . . . . Journal of High Energy Physics

Nucl. Instrum. Meth. . . . . . Nuclear Instruments and Methods in Physics Research

Phys. Lett. . . . . . . . . . . . . . . . . . . Physics Letters

Phys. Rev. . . . . . . . . . . . . The Physical Review 


\section{Bibliography}

[1] L. Wang et al., "Measurement of the Shape of the Boson Transverse Momentum distribution using $p \bar{p} \rightarrow Z / \gamma^{*} \rightarrow e e$ events at the Tevatron Collider" (2007), DØ note 5187.

[2] "Precision Electroweak Measurements and Constraints on the Standard Model: Draft 2.0" (2009), SLAC-PUB-13830.

[3] M. Cacciari, S. Frixione, M. L. Mangano, P. Nason, and G. Ridolfi, "Updated predictions for the total production cross sections of top and of heavier quark pairs at the Tevatron and at the LHC", JHEP 09, 127 (2008), 0804.2800.

[4] V. M. Abazov et al. (DØ), "The Upgraded DØ Detector”, Nucl. Instrum. Meth. A565, 463 (2006), physics/0507191.

[5] V. M. Abazov et al. (DØ), "Run IIb upgrade technical design report" (2002), FERMILAB-PUB-02-327-E.

[6] C. Amsler et al. (Particle Data Group), "Review of particle physics", Phys. Lett. B667, 1 (2008).

[7] G. Borissov, "Technical Details of AA Tracking" (2005), Tracking Workshop, Dec 6. 2005.

[8] A. Khanov, "HTF: Histogramming Method for Finding Tracks. The Algorithm Description" (2000), DØ note 3778. 
[9] A. Juste, "Jet Energy Scale Determination at DØ RunII (final p17 version)" (2007), DØ note 5382.

[10] D. Boline, "Sample Dependent Correction to the Jet Energy Scale" (2008), DØ note 5572 .

[11] C. Ochando and J. Grivaz, "SSR for p17" (2008), DØ note 5609.

[12] D. Boline, "Jet Multiplicity Dependent Reweighting of the Z Boson $p_{T}$ in p20" (2008), DØ note 5569.

[13] "Combination of CDF and D0 Results on the Mass of the Top Quark" (2009), 0903.2503.

[14] S. Moch and P. Uwer, "Theoretical status and prospects for top-quark pair production at hadron colliders", Phys. Rev. D78, 034003 (2008), 0804.1476.

[15] V. M. Abazov et al. (D0), "Combination of ttbar cross section measurements and constraints on the mass of the top quark and its decays into charged Higgs bosons", Phys. Rev. D80, 071102 (2009), 0903.5525.

[16] U. Bassler and G. Bernardi, "Towards a Coherent Treatment of Calorimetric Energies: Missing Transverse Energy, Jets, E.M. Objects and the T42 Algorithm" (2002), DØ note 4124 .

[17] J. Vlimant et al., "Technical description of the T42 Algorithm for the calorimeter noise suppression" (2003), DØ note 4146.

[18] G. Bernardi et al., "Improvements from the T42 Algorithm on Calorimeter Objects Reconstruction" (2004), DØ note 4335.

[19] V. M. Abazov et al., "The muon system of the Run II D $\varnothing$ detector", Nucl. Instrum. Meth. A552, 372 (2005), physics/0503151.

[20] J. Butler, "Local Muon Momentum Resolution" (2002), DØ note 4002. 
[21] G. Hasketh, "Central track extrapolation through the DØ detector" (2003), DØ note 4079 .

[22] S. Agostinelli et al. (GEANT4), "GEANT4: A simulation toolkit", Nucl. Instrum. Meth. A506, 250 (2003).

[23] M. Arthaud et al., "Muon Momentum Oversmearing for p17 Data" (2007), DØ note 5444 .

[24] M. Arthaud et al., "Muon Momentum Oversmearing for p20 Data" (2007), DØ note 5449 .

[25] M. Narain and U. Heintz, "A likelihood test for electron id" (1994), DØ note 2386.

[26] J. Kozminski et al., "Electron likelihood in p14" (2004), DØ note 4449.

[27] M. Aoki, "Electron likelihood in p20" (2008), DØ note 5675.

[28] G. C. Blazey et al., "Run II jet physics" (2000), hep-ex/0005012.

[29] E. Busato, "Jet Algorithms in the DØ Run II Software: Description and User's Guide" (2004), DØ note 4457.

[30] K. DeVaughan et al., "Jet Energy Scale Determination for DØ RunIIb (final p20 version)" (2008), DØ note 5801.

[31] D. E. Groom, "Energy flow in a hadronic cascade: Application to hadron calorimetry", Nucl. Instrum. Meth. A572, 633 (2007), physics/0605164.

[32] N. Makovec and J. Grivaz, "Shifting, Smearing, and Removing Simulated Jets" (2005), DØ note 4914.

[33] M. L. Mangano, M. Moretti, F. Piccinini, R. Pittau, and A. D. Polosa, "ALPGEN, a generator for hard multiparton processes in hadronic collisions", JHEP 07, 001 (2003), hep-ph/0206293. 
[34] T. Sjostrand et al., "High-energy physics event generation with PYTHIA 6.1", Comput. Phys. Commun. 135, 238 (2001), hep-ph/0010017.

[35] S. Hoche et al., "Matching parton showers and matrix elements" (2006), hep-ph/0602031.

[36] D. Gillberg, "Heavy Flavour Removal and Determination of Weighting Factors for ALPGEN $W+$ jets Monte Carlo" (2006), DØ note 5129.

[37] S. Jadach, J. H. Kuhn, and Z. Was, "TAUOLA: A Library of Monte Carlo programs to simulate decays of polarized tau leptons", Comput. Phys. Commun. 64, 275 (1990).

[38] M. Jezabek, Z. Was, S. Jadach, and J. H. Kuhn, "The tau decay library TAUOLA, update with exact $\mathrm{O}$ (alpha) QED corrections in tau $\rightarrow$ mu. (e) neutrino anti-neutrino decay modes", Comput. Phys. Commun. 70, 69 (1992).

[39] D. J. Lange, "The EvtGen particle decay simulation package", Nucl. Instrum. Meth. A462, 152 (2001).

[40] E. Barberio, B. van Eijk, and Z. Was, "PHOTOS: A Universal Monte Carlo for QED radiative corrections in decays", Comput. Phys. Commun. 66, 115 (1991).

[41] E. Barberio and Z. Was, "PHOTOS: A Universal Monte Carlo for QED radiative corrections. Version 2.0", Comput. Phys. Commun. 79, 291 (1994).

[42] R. Brun, R. Hagelberg, M. Hansroul, and J. C. Lassalle, "GEANT: SIMULATION PROGRAM FOR PARTICLE PHYSICS EXPERIMENTS. USER GUIDE AND REFERENCE MANUAL" (1978), CERN-DD-78-2-REV.

[43] T. B. M. Shamim, "Generator Level Reweighting of Z boson $p_{T}$ " (2008), DØ note 5565.

[44] H. Schellman, "Update to Beam z shape for RunIIb" (2007), DØ note 5540.

[45] J. Hegeman, "Luminosity determination and reweighting of Monte Carlo overlay luminosity for p17 hadronic top analyses" (2008), DØ note 5561. 
[46] P. Calfayan et al., "Muon identification certification for p17 data" (2007), DØ note 5157.

[47] S. Cho et al., "Muon id certification for p20 data" (2008), DØ note 5824.

[48] P. Gris, "Electron Smearing Studies with RunIIa Data" (2007), DØ note 5400.

[49] F. Deliot et al., "Trigger efficiency measurement for or of electron muon triggers" (2008), DØ note 5587.

[50] P. Calfayan et al., "ORing single muon triggers in p17 data" (2008), DØ note 5329.

[51] B. Martin et al., "Final measurement of the $t \bar{t}$ production cross-section at $\sqrt{s}=1.96$ $\mathrm{TeV}$ in the ee final state using p17 data set" (2008), DØ note 5579.

[52] M. Arthaud et al., "Final Measurement of the $t \bar{t}$ Production Cross-section at $\sqrt{s}=$ $1.96 \mathrm{TeV}$ in the Electron Muon Final State using p17 data set” (2008), DØ note 5580.

[53] U. Bassler et al., "Measurement of the $t \bar{t}$ Production Cross-section at $\sqrt{s}=1.96 \mathrm{TeV}$ in the Dimuon Final State using RunIIa data set" (2008), DØ note 5581.

[54] C. Tully and R. Wagner, " $t \bar{t}$ Cross Section in the Lepton+Track Channel with $1 \mathrm{fb}^{-1}$ " (2007), DØ note 5421.

[55] L. Sonnenschein, "Algebraic approach to solve t anti-t dilepton equations", Phys. Rev. D72, 095020 (2005), hep-ph/0510100.

[56] L. Sonnenschein, "Analytical solution of t anti-t dilepton equations", Phys. Rev. D73, 054015 (2006), hep-ph/0603011.

[57] R. H. Dalitz and G. R. Goldstein, "The Decay and polarization properties of the top quark", Phys. Rev. D45, 1531 (1992).

[58] Z. Ye et al., "Measurement of the Top Quark Mass in the Lepton+Jets Final State of Top Quark Pair Production Using Matrix Element Method on 2.6 fb1 DØRunIIb Data" (2009), DØ note 5853. 
[59] Y. Peters et al., "Reweighting of the fragmentation function for the DØ Monte Carlo" (2007), DØ note 5325.

[60] P. Skands and D. Wicke, "Non-perturbative QCD effects and the top mass at the Tevatron", Eur. Phys. J. C52, 133 (2007), hep-ph/0703081.

[61] H. L. Lai et al. (CTEQ), "Global QCD analysis of parton structure of the nucleon: CTEQ5 parton distributions", Eur. Phys. J. C12, 375 (2000), hep-ph/9903282.

[62] D. Stump et al., "Inclusive jet production, parton distributions, and the search for new physics", JHEP 10, 046 (2003), hep-ph/0303013.

[63] V. M. Abazov et al. (D0), "Measurement of the top quark mass in final states with two leptons", Phys. Rev. D80, 092006 (2009), 0904.3195.

[64] T. D. Collaboration, "Combination of the D $\varnothing$ top quark mass measurements" (2009), DØ note 5900-CONF.

[65] V. M. Abazov et al. (D0), "A precision measurement of the mass of the top quark", Nature 429, 638 (2004), hep-ex/0406031.

[66] P. Avery, "Combining Measurements with Correlated Errors" (1996), CBX 95-55. 


\title{
CURRICULUM VITAE
}

\author{
Daniel Boline
}

Work: (630) 840-2931
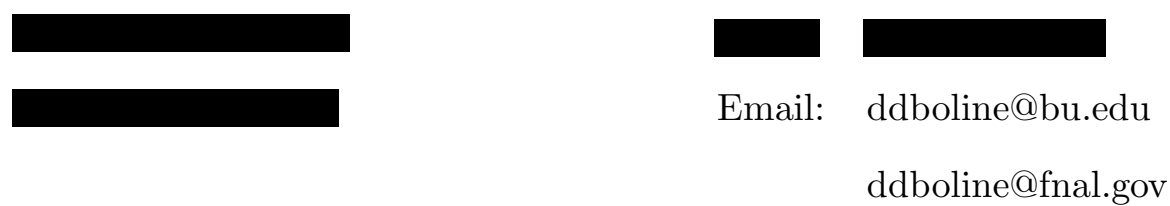

\section{Education}

- Ph.D., Physics, Boston University, Boston MA (Expected Jan 26, 2010)

- B.S., Physics, Michigan Technological University, Houghton MI (2002)

$\bullet$

\section{Employment}

- 2004 - Present: Research Assistant, Department of Physics, Boston University Supervisor: Ulrich Heintz (401) 863-6817

590 Commonwealth Ave, Boston, MA 02215

- 2002 - 2004: Teaching Assistant, Department of Physics, Boston University 590 Commonwealth Ave, Boston, MA 02215

- 2002: Research Assistant, Department of Physics, Michigan Technological University Supervisior: Bryan Suits (906) 487-2093 1400 Townsend Drive, Houghton, Michigan 49931-1295

\section{Professional Experience}

- 2004 - Present: Research Assistant, Department of Physics, Boston University 
- Measurement of Top Quark Mass in the Dilepton Channel with the MWT method.

* At the moment this measurement is the most precise in the dilepton channel at $\mathrm{D} \varnothing$. I wrote the analysis code, ran the analysis, sought to optimize the event selection and measurement technique, evaluated systematics, and did many studies to understand discrepancies.

- Sample Dependence Correction to Jet Energy Scale.

* This correction was intended to remove a $\sim 1 \%$ bias on the jet energy scale calibration. I wrote code to measure this bias in an arbitrary physics sample (e.g. $t \bar{t}$ ) and to apply the derived correction to that sample. I then used this code to derive such a correction for a $t \bar{t}$ sample, and investigated various possible issues with the correction.

- Certification of Secondary Vertex Tagging Algorithm.

* Derived fake rates and tagging efficiencies for data and monte carlo, and scale factors to correct monte carlo to data. Then applied these derived corrections onto calibration samples, to verify the validity of the tagging rates, fake rates, and scale factors.

\section{Teaching}

- 2002 - 2004: Teaching Assistant, Department of Physics, Boston

\section{Computer Skills}

- Fortran (77,90), C, C++, Latex, Python, ROOT, Unix/Linux Shell Programming

- Linux System Administration (Scientific Linux, Ubuntu) 


\section{Contributions to Conferences}

- APS April Meeting, Jacksonville, Fl, 2008, "Measurement of the Top Quark Mass at D0 Using the Matrix Weighting Method on Dilepton Events"

- DPF, Detroit MI, 2009, "Top Mass Measurements with the D0 Detector"

\section{Publications}

- "Top Mass in Dilepton Channel", D. Boline, Doctoral Thesis, Boston University (to be submitted 2009).

- "Measurement of the top quark mass in final states with two leptons", (2009) hep-ex/0904.3195, Submitted to PRD.

- Measurement of the top quark mass in the dilepton channel, Phys. Lett. B 655, 7 (2007).

- "Measurement of the Top Quark Mass in the Dilepton Channel at D0 Using the Matrix Weighting Method at DØwith $3 \mathrm{fb}^{-1}$ of RunIIb Data", D. Boline, U. Heintz, DØ note 5988 (2009).

- "Measurement of the Top Quark Mass in the Dilepton Channel at D0", D. Boline, U. Heintz, DØ note 5678 (2008).

- "Sample Dependent Correction to the Jet Energy Scale", D. Boline, DØ note 5572 (2008).

- "Jet Multiplicity Dependent Reweighting of the Z Boson $p_{T}$ in p20", D. Boline, DØ note 5569 (2008).

- "Update on $b$-quark jet identification with Secondary Vertex reconstruction using p14-Pass2 Samples", D. Boline, , L. Feligioni, M.Narain, DØ note 4796 (2005). 